DOI 10.4171/JEMS/420

John L. Lewis · Kaj Nyström · Andrew Vogel



\title{
On the dimension of $p$-harmonic measure in space
}

Received June 17, 2011

\begin{abstract}
Let $\Omega \subset \mathbb{R}^{n}, n \geq 3$, and let $p, 1<p<\infty, p \neq 2$, be given. In this paper we study the dimension of $p$-harmonic measures that arise from nonnegative solutions to the $p$-Laplace equation, vanishing on a portion of $\partial \Omega$, in the setting of $\delta$-Reifenberg flat domains. We prove, for $p \geq n$, that there exists $\tilde{\delta}=\tilde{\delta}(p, n)>0$ small such that if $\Omega$ is a $\delta$-Reifenberg flat domain with $\delta<\tilde{\delta}$, then $p$-harmonic measure is concentrated on a set of $\sigma$-finite $H^{n-1}$-measure. We prove, for $p \geq n$, that for sufficiently flat Wolff snowflakes the Hausdorff dimension of $p$-harmonic measure is always less than $n-1$. We also prove that if $2<p<n$, then there exist Wolff snowflakes such that the Hausdorff dimension of $p$-harmonic measure is less than $n-1$, while if $1<p<2$, then there exist Wolff snowflakes such that the Hausdorff dimension of $p$-harmonic measure is larger than $n-1$. Furthermore, perturbing off the case $p=2$, we derive estimates for the Hausdorff dimension of $p$-harmonic measure when $p$ is near 2 .
\end{abstract}

Keywords. $p$-harmonic function, $p$-harmonic measure, Hausdorff dimension, Reifenberg flat domain, Wolff snowflake

\section{Introduction}

Let $G \subset \mathbb{R}^{n}$ be a bounded domain. Recall that the solution in $G$ to the Dirichlet problem for the Laplace operator with continuous boundary data $f$ is the unique smooth function $u$ which is harmonic in $G$ and equals $f$ on $\partial G$. The maximum principle and Riesz representation theorem yield, for sufficiently regular domains, the formula

$$
u(x)=\int_{\partial G} f(y) d \omega^{x}(y) \quad \text { whenever } x \in G,
$$

where $\omega=\omega^{x}$ is referred to as the harmonic measure at $x$ associated to the Laplace operator. Let also $g=g(\cdot)=g(\cdot, x)$ be the Green function for $G$ with pole at $x \in G$ and

J. L. Lewis: Department of Mathematics, University of Kentucky, Lexington, KY 40506-0027, USA; e-mail: john@ms.uky.edu

K. Nyström: Department of Mathematics, Uppsala University, S-751 06 Uppsala, Sweden; e-mail: kaj.nystrom@math.uu.se

A. Vogel: Department of Mathematics, Syracuse University, Syracuse, NY 13244, USA; e-mail: alvogel@syracuse.edu

Mathematics Subject Classification (2010): Primary 35J25, 35J70 
extend $g$ to $\mathbb{R}^{n} \backslash G$ by putting $g \equiv 0$ on $\mathbb{R}^{n} \backslash G$. Then $\omega$ is the Riesz measure associated to $g$ in the sense that

$$
\int\langle\nabla g, \nabla \phi\rangle d x=-\int \phi d \omega \quad \text { whenever } \phi \in C_{0}^{\infty}\left(\mathbb{R}^{n} \backslash\{x\}\right) .
$$

We define the Hausdorff dimension of $\omega$, denoted $\mathrm{H}-\operatorname{dim} \omega$, by

H-dim $\omega=\inf \left\{\alpha\right.$ : there exists $E$ Borel $\subset \partial G$ with $H^{\alpha}(E)=0$ and $\left.\omega(E)=\omega(\partial G)\right\}$,

where $H^{\alpha}(E)$, for $\alpha \in \mathbb{R}_{+}$, is the $\alpha$-dimensional Hausdorff measure of $E$ defined below. In the past quarter century a number of remarkable results concerning H-dim $\omega$ have been established in planar domains, $\Omega \subset \mathbb{R}^{2}$. In particular, Carleson [C] showed that $\mathrm{H}-\operatorname{dim} \omega=1$ when $\partial \Omega$ is a snowflake and that $\mathrm{H}-\operatorname{dim} \omega \leq 1$ for any self-similar Cantor set. Later Makarov [M] proved that $\mathrm{H}-\operatorname{dim} \omega=1$ for any simply connected domain in the plane. Jones and Wolff [JW] proved that $\mathrm{H}-\operatorname{dim} \omega \leq 1$ whenever $\Omega \subset \mathbb{R}^{2}$ and $\omega$ exists, and Wolff [W2] strengthened [JW] by showing that $\omega$ is concentrated on a set of $\sigma$-finite $H^{1}$-measure. We also mention results of Batakis [Ba], Kaufmann-Wu [KW], and Volberg [V] who showed, for certain fractal domains and domains whose complements are Cantor sets, that

$$
\text { Hausdorff dimension of } \partial \Omega=\inf \left\{\alpha: H^{\alpha}(\partial \Omega)=0\right\}>\mathrm{H}-\operatorname{dim} \omega \text {. }
$$

In $\mathbb{R}^{n}, n \geq 3$, results for the dimension of harmonic measure can be found in [Bo], [W2], [LVV], [KT], [KPT] but we emphasize that harmonic measure in space is, in general, considerably less understood than harmonic measure in the plane. Indeed, Wolff [W2] showed in three dimensions that there is "no reason at all" for the Hausdorff dimension of harmonic measure to be equal to two, in contrast to the theorem of Makarov.

For $p \neq 2, p$-harmonic measure is a generalization, to the setting of the $p$-Laplace operator, of the notion of harmonic measure. Results concerning the Hausdorff dimension of $p$-harmonic measures in the plane can be found in [BL], [L] and [LNP]. Some of these results are presented below as Theorems A-D.

In this paper we focus on the Hausdorff dimension of $p$-harmonic measure, $p \neq 2$, in domains $\Omega \subset \mathbb{R}^{n}$ with $n \geq 3$. To the best of our knowledge, this is the first paper in the literature devoted to the Hausdorff dimension of $p$-harmonic measure, $p \neq 2$, in the higher dimensional setting. The nonlinearity and degeneracy of the $p$-Laplacian when $p \neq 2$, has made such a study difficult. Only recently have the first and second author developed the tools that make such a study feasible (see [LN1]-[LN6] and [LLuN]). In these papers the tools developed were used to solve several longstanding research problems on $p$-harmonic functions.

To proceed we introduce some notation. Points in Euclidean $n$-space $\mathbb{R}^{n}$ are denoted by $x=\left(x_{1}, \ldots, x_{n}\right)$ or $\left(x^{\prime}, x_{n}\right)$ where $x^{\prime}=\left(x_{1}, \ldots, x_{n-1}\right) \in \mathbb{R}^{n-1}$. We let $\bar{E}, \partial E$, diam $E$ be the closure, boundary, and diameter of the set $E \subset \mathbb{R}^{n}$ and we define $d(y, E)$ to be the distance from $y \in \mathbb{R}^{n}$ to $E ;\langle\cdot, \cdot\rangle$ denotes the standard inner product on $\mathbb{R}^{n}$ and 
we let $|x|=\langle x, x\rangle^{1 / 2}$ be the Euclidean norm of $x ; B(x, r)=\left\{y \in \mathbb{R}^{n}:|x-y|<r\right\}$ for $x \in \mathbb{R}^{n}, r>0$, and $d x$ denotes Lebesgue $n$-measure on $\mathbb{R}^{n}$. Let

$$
h(E, F)=\max (\sup \{d(y, E): y \in F\}, \sup \{d(y, F): y \in E\})
$$

be the Hausdorff distance between the sets $E, F \subset \mathbb{R}^{n}$. If $O \subset \mathbb{R}^{n}$ is open and $1 \leq$ $q \leq \infty$, then by $W^{1, q}(O)$ we denote the space of equivalence classes of functions $f$ with distributional gradient $\nabla f=\left(f_{x_{1}}, \ldots, f_{x_{n}}\right)$, both of which are $q$ th power integrable on $O$. Let $\|f\|_{1, q}=\|f\|_{q}+\||\nabla f|\|_{q}$ be the norm in $W^{1, q}(O)$ where $\|\cdot\|_{q}$ denotes the usual Lebesgue $q$-norm in $O$. Next let $C_{0}^{\infty}(O)$ be the set of infinitely differentiable functions with compact support in $O$ and let $W_{0}^{1, q}(O)$ be the closure of $C_{0}^{\infty}(O)$ in the norm of $W^{1, q}(O)$.

Given $\lambda$ a nondecreasing positive function on $\left(0, r_{0}\right), r_{0}>0$, with $\lambda(0)=0$, we next define Hausdorff measure corresponding to $\lambda$ (denoted $H^{\lambda}$ ) as follows. For fixed $\delta$ with $0<\delta<r_{0}$, and $E \subseteq \mathbb{R}^{n}$, let $L(\delta)=\left\{B\left(x_{i}, r_{i}\right)\right\}$ be a set of balls such that $E \subseteq \bigcup B\left(x_{i}, r_{i}\right)$ and $0<r_{i}<\delta$ for $i=1,2, \ldots$. Set

$$
\phi_{\delta}^{\lambda}(E)=\inf _{L(\delta)}\left(\sum \lambda\left(r_{i}\right)\right) .
$$

Then

$$
H^{\lambda}(E)=\lim _{\delta \rightarrow 0} \phi_{\delta}^{\lambda}(E)
$$

Let $\alpha(k)$ denote the volume of the unit ball in $\mathbb{R}^{k}$. In case $\lambda(r)=\alpha(k) r^{k}$ we write $H^{k}$ for $H^{\lambda}$, and call it $k$-dimensional Hausdorff measure in $\mathbb{R}^{n}$.

Given a bounded domain $G$, we say that $u$ is $p$-harmonic in $G$ provided $u \in W^{1, p}(G)$ and

$$
\int|\nabla u|^{p-2}\langle\nabla u, \nabla \theta\rangle d x=0
$$

whenever $\theta \in W_{0}^{1, p}(G)$. Observe that if $u$ is smooth and $\nabla u \neq 0$ in $G$, then

$$
\nabla \cdot\left(|\nabla u|^{p-2} \nabla u\right) \equiv 0 \quad \text { in } G
$$

and $u$ is a classical solution in $G$ to the $p$-Laplace partial differential equation. Here and below, $\nabla \cdot$ is the divergence operator.

Let $\Omega \subset \mathbb{R}^{n}$ be a domain, and suppose that $\partial \Omega$ is bounded and nonempty. Let $N$ be a neighborhood of $\partial \Omega$, let $p$ be fixed, $1<p<\infty$, and let $u$ be a positive weak solution to the $p$-Laplace equation in $\Omega \cap N$. Assume that $u$ has zero boundary values on $\partial \Omega$ in the Sobolev sense. More specifically if $\zeta \in C_{0}^{\infty}(N)$, then $u \zeta \in W_{0}^{1, p}(\Omega \cap N)$. Extend $u$ to $N \backslash \Omega$ by putting $u \equiv 0$ on $N \backslash \Omega$. Then $u \in W^{1, p}(N)$ and it follows from (1.1), as in $[\mathrm{HKM}]$, that there exists a positive finite Borel measure $\mu$ on $\mathbb{R}^{n}$, with support contained in $\partial \Omega$, satisfying

$$
\int|\nabla u|^{p-2}\langle\nabla u, \nabla \phi\rangle d x=-\int \phi d \mu
$$

for all $\phi \in C_{0}^{\infty}(N)$. If $\partial \Omega$ is smooth enough, then

$$
d \mu=|\nabla u|^{p-1} d H^{n-1} \quad \text { on } \partial \Omega .
$$


In the case $p=2$, and if $u$ is the Green function for $\Omega$ with pole at $x \in \Omega$, then the measure $\mu$ coincides with harmonic measure at $x, \omega=\omega^{x}$, introduced above. We will refer to $\mu$ as the $p$-harmonic measure associated to $u$. The Hausdorff dimension of the measure $\mu$, denoted $\mathrm{H}-\operatorname{dim} \mu$, is defined as

$$
\begin{array}{r}
\mathrm{H}-\operatorname{dim} \mu=\inf \left\{\alpha: \text { there exists } E \text { Borel } \subset \partial \Omega \text { with } H^{\alpha}(E)=0\right. \\
\text { and } \mu(E)=\mu(\partial \Omega)\} .
\end{array}
$$

As mentioned above, in [BL], [L] and [LNP] the Hausdorff dimension of $p$-harmonic measure $\mu$ is studied for general $p, 1<p<\infty$ and $n=2$. Theorems A and B stated below are proved in [BL] and [L], respectively, while Theorems $\mathrm{C}$ and $\mathrm{D}$ are proved in [LNP]. In fact, Theorem D is a corollary to Theorem C (see [LNP]). For the definition of a $k$-quasi-circle we refer to [L].

Theorem A. Let $p \in(1, \infty), p \neq 2$, and let $u, \mu$ be as in (1.1), (1.3). If $\partial \Omega$ is a quasicircle, then $\mathrm{H}-\operatorname{dim} \mu \leq 1$ for $2<p<\infty$, and $\mathrm{H}$-dim $\mu \geq 1$ for $1<p<2$. Moreover, if $\partial \Omega$ is the von Koch snowflake then strict inequality holds for $\mathrm{H}-\operatorname{dim} \mu$.

Theorem B. Let $p \in(1, \infty), p \neq 2$, and let $u, \mu$ be as in (1.1), (1.3). Then there exists $k_{0}(p)>0$ such that if $\partial \Omega$ is a $k$-quasi-circle and $0<k<k_{0}(p)$, then:

(a) $\mu$ is concentrated on a set of $\sigma$-finite $H^{1}$-measure when $p>2$.

(b) There exists $A=A(p)$ with $0<A(p)<\infty$ such that if $1<p<2$, then $\mu$ is absolutely continuous with respect to Hausdorff measure defined relative to $\tilde{\lambda}$ where

$$
\tilde{\lambda}(r)=r \exp [A \sqrt{\log 1 / r \log \log \log 1 / r}], \quad 0<r<10^{-6} .
$$

Theorem C. Let $p \in(1, \infty), p \neq 2$, and let $u, \mu$ be as in (1.1), (1.3). Suppose that $\Omega$ is simply connected. Put

$$
\lambda(r)=r \exp [A \sqrt{\log 1 / r \log \log 1 / r}], \quad 0<r<10^{-6} .
$$

Then the following is true.

(a) If $p>2$, then there exists $A=A(p) \leq-1$ such that $\mu$ is concentrated on a set of $\sigma$-finite $H^{\lambda}$-measure.

(b) If $1<p<2$, then there exists $A=A(p) \geq 1$ such that $\mu$ is absolutely continuous with respect to $H^{\lambda}$.

Theorem D. Let $p \in(1, \infty), p \neq 2$, and let $u, \mu$ be as in (1.1), (1.3). Suppose that $\Omega$ is simply connected. Then $\mathrm{H}-\operatorname{dim} \mu \leq 1$ for $2<p<\infty$, and $\mathrm{H}-\operatorname{dim} \mu \geq 1$ for $1<p<2$.

For results in $\mathbb{R}^{3}$ we recall the famous paper [W2] where Wolff showed how to construct domains, which we call Wolff snowflakes, for which the corresponding harmonic measure had Hausdorff dimension either greater than 2 or less than 2. Also, in [LVV], it was shown that the Hausdorff dimension of harmonic measures on both sides of a Wolff snowflake in $\mathbb{R}^{n}$ can have dimension either $>n-1$ or $<n-1$. So for $n \geq 3$ the Hausdorff dimension of harmonic measure in $\mathbb{R}^{n}$ may not be equal to $n-1$.

In this paper we prove Theorems $1-4$, concerning the Hausdorff dimension of $p$ harmonic measure, for $1<p<\infty, p \neq 2$, in certain subsets $\Omega \subset \mathbb{R}^{n}, n \geq 3$. In Theorem 1 we prove that (a) of Theorem B holds for $p$-harmonic measure in sufficiently 
flat Reifenberg flat domains $\subset \mathbb{R}^{n}, n \geq 3$, when $p \geq n$. Reifenberg flat domains are defined in Definition 2.1. In Theorem 2 we prove, for $n \geq 3$, that if $2<p<n$, then there exist Wolff snowflakes such that $\mathrm{H}-\operatorname{dim} \mu<n-1$, while if $1<p<2$, then there exist Wolff snowflakes such that $\mathrm{H}-\operatorname{dim} \mu>n-1$. In addition, we prove that if $p \geq n$ then we always get, in the setting of the construction outlined, Wolff snowflakes for which H-dim $\mu<n-1$. As outlined below, the sign of the difference $(n-1)-$ H-dim $\mu$ is determined by the sign of a certain entropy integral for $p$-harmonic measure and this connection is established in Theorem 3. Finally, using [W2], [LVV], and perturbing off the $p=2$ case we show that there are Wolff snowflakes such that the sign of $(n-1)-\mathrm{H}$-dim $\mu$ equals the sign of $(n-1)-\mathrm{H}$-dim $\omega$, for $p$ near 2 (see Theorem 4$)$.

The rest of this paper is organized as follows. In Section 2 we state our Theorems 1-4 and briefly outline the proof of Theorems 1-2. In Section 3 we first state some basic estimates for $p$-harmonic functions and $p$-harmonic measure in nontangentially accessible, Reifenberg flat, and Lipschitz domains. In Section 4 we list some lemmas for elliptic partial differential equations whose degeneracy is given in terms of an $A_{2}$-weight. Theorem 1 is proved in Section 5 while Sections 6 and 7 are devoted to the proof of Theorems 2-4.

\section{Statement of main results: Theorems 1-4}

Definition 2.1. Let $\Omega \subset \mathbb{R}^{n}$ be a domain. Then $\Omega$ and $\partial \Omega$ are said to be $\left(\delta, r_{0}\right)$-Reifenberg flat if for all $w \in \partial \Omega$ and $0<r<r_{0}$, there exists a hyperplane $P=P(w, r)$ containing $w$ such that

(a) $h(\partial \Omega \cap B(w, r), P \cap B(w, r)) \leq \delta r$,

(b) $\{x \in \Omega \cap B(w, r / 2): d(x, \partial \Omega) \geq 2 \delta r\}$ is contained in one component of $\mathbb{R}^{n} \backslash P$.

We will say that $\Omega$ and $\partial \Omega$ are $\delta$-Reifenberg flat if they are $\left(\delta, r_{0}\right)$-Reifenberg flat for some $r_{0}>0$. In our construction of Wolff snowflake domains we will have $r_{0}=\infty$. As in [KT] one can show that there is a small positive $\hat{\delta}=\hat{\delta}(n)$ such that any $\delta$-Reifenberg flat domain with $0<\delta<\hat{\delta}$ is an NTA-domain with $M=M(n)$. For the definition of NTA-domains we refer to Definition 3.1 below.

To give examples of classes of $\left(\delta, r_{0}\right)$-Reifenberg flat domains we first note that if $\Omega \subset \mathbb{R}^{n}$ is a Lipschitz domain with constant $M$, then $\Omega$ is $\left(\delta, r_{0}\right)$-Reifenberg flat for some $\delta=\delta(M), r_{0}>0$ with $\delta(M) \rightarrow 0$ as $M \rightarrow 0$. However, using [LN3] we see that in the setting of Lipschitz domains we always have $\mathrm{H}$-dim $\mu=n-1$ and hence this class of examples is less interesting from the perspective of Theorem 1. To give other examples, we say, following [LLuN], that $\Omega$ is a quasi-ball provided $\Omega=f(B(0,1))$, where $f=\left(f_{1}, \ldots, f_{n}\right): \mathbb{R}^{n} \rightarrow \mathbb{R}^{n}$ is a $K>1$ quasi-conformal mapping of $\mathbb{R}^{n}$ onto $\mathbb{R}^{n}$. That is, $f_{i} \in W^{1, n}(B(0, \rho)), 0<\rho<\infty, 1 \leq i \leq n$, and for almost every $x \in \mathbb{R}^{n}$ with respect to Lebesgue $n$-measure the following hold:

$$
\begin{gathered}
|D f(x)|^{n}=\sup _{|h|=1}|D f(x) h|^{n} \leq K\left|J_{f}(x)\right|, \\
J_{f}(x) \geq 0 \quad \text { or } \quad J_{f}(x) \leq 0 .
\end{gathered}
$$


In this display we have written $D f(x)=\left(\partial f_{i} / \partial x_{j}\right)$ for the Jacobian matrix of $f$, and $J_{f}(x)$ for the Jacobian determinant of $f$ at $x$. If $\Omega=f(B(0,1))$ where $f$ is a $K$ quasiconformal mapping of $\mathbb{R}^{n}$ onto $\mathbb{R}^{n}$, then one can show that $\partial \Omega$ is $\delta$-Reifenberg flat, with $r_{0}=1$, where $\delta \rightarrow 0$ as $K \rightarrow 1$ (see [R, Theorems 12.5-12.7]). For more on $\delta$-Reifenberg flat domains we refer to [CKL].

We now state our first main result.

Theorem 1. Let $0<\delta<\hat{\delta}(n)$ and suppose $\Omega \subset \mathbb{R}^{n}, n \geq 3$, is a $\delta$-Reifenberg flat domain with $r_{0}=\infty$. Fix $p \geq n$ and let $u, \mu$ be as in (1.1), (1.3). There exists $\tilde{\delta}=\tilde{\delta}(p, n)>0$ such that if $0<\delta \leq \tilde{\tilde{\delta}}$, then $\mu$ is concentrated on a set of $\sigma$-finite $H^{n-1}$-measure.

Remark 2.2. Note that if $\Omega$ is $\delta$-Reifenberg flat, then $\partial \Omega$ need not have locally finite $H^{n-1}$-measure or a tangent plane at any boundary point. However, one can show, as in [LN5], that given $\eta>0$ there exists $\delta>0$ such that if $\Omega$ is a $\delta$-Reifenberg flat domain, then $H^{n-1+\eta}(\partial \Omega \cap B(w, r))<\infty$, whenever $w \in \partial \Omega$ and $r>0$. This inequality clearly implies that $\mathrm{H}-\operatorname{dim} \mu \leq n-1+\eta$. However, Theorem 1 is not implied by this estimate.

Outline of the proof of Theorem 1. The proof of Theorem 1 appears in Section 5. We first observe, using the basic estimates of Section 3, that it suffices to prove Theorem 1 when $u>0$ is $p$-harmonic in $\Omega$ with continuous boundary values 0 on $\partial \Omega$. To state some results from [LN5], let $\Omega$ be a $\delta$-Reifenberg flat domain with $r_{0}=\infty, 1<p<\infty$, and let $u>0$ be a $p$-harmonic function in $\Omega$ with $u \equiv 0$ on $\partial \Omega$ in the sense of continuous boundary values. Then there exist $\delta_{0}>0$ and $c_{1} \geq 1$, depending only on $p, n$, such that if $0<\delta \leq \delta_{0}$ and $x \in \Omega$, then $u$ is infinitely differentiable in $\Omega\left(u \in C^{\infty}(\Omega)\right)$ and

$$
\begin{aligned}
& c_{1}^{-1}|\nabla u(x)| \leq u(x) / d(x, \partial \Omega) \leq c_{1}|\nabla u(x)|, x \in \Omega, \\
& |\nabla u|^{p-2} \text { extends to an } A_{2} \text {-weight on } \mathbb{R}^{n} \text { with constant } \leq c_{1} .
\end{aligned}
$$

For the definition of an $A_{2}$-weight and its $A_{2}$-constant, see Section 4. Next consider the partial differential equation

$$
L \zeta(x)=\sum_{i, k=1}^{n} \frac{\partial}{\partial x_{i}}\left[b_{i k}(x) \zeta_{x_{k}}(x)\right]=0,
$$

where at $x \in \Omega$,

$$
b_{i k}(x)=|\nabla u|^{p-4}\left[(p-2) u_{x_{i}} u_{x_{k}}+\delta_{i k}|\nabla u|^{2}\right](x), \quad 1 \leq i, k \leq n,
$$

and $\delta_{i k}$ is the Kronecker $\delta$. From (2.6) and smoothness of $u$ we see that $b_{i k}$ are infinitely differentiable in $\Omega$ and, at $x \in \Omega$,

$$
\min \{p-1,1\}|\xi|^{2}|\nabla u(x)|^{p-2} \leq \sum_{i, k=1}^{n} b_{i k} \xi_{i} \xi_{k} \leq \max \{1, p-1\}|\nabla u(x)|^{p-2}|\xi|^{2} .
$$

From (2.6), Lemma 3.5, and Harnack's inequality for $u$ it follows that $\left(b_{i k}(x)\right)$ are locally uniformly elliptic in $\Omega$ with ellipticity constants given in terms of an $A_{2}$-weight on $\mathbb{R}^{n}$. 
Also, as in [LN1, (1.5)-(1.8)], it follows that $u, u_{x_{k}}, 1 \leq k \leq n$, are all classical solutions to $L$. In Lemma 5.1 we show that $\zeta=\log |\nabla u|$ is a subsolution to (2.7) when $p \geq n$. Using these facts, we can then use the argument in [L], with minor modifications, to get Theorem 1.

To outline this argument, we note that Makarov (see [P, Theorem 6.13]) proved a similar result in $\mathbb{R}^{2}$ for harmonic measure $(p=2)$ in a simply connected domain using Plessner's theorem. Moreover, the main step in proving Plessner's theorem is to show that if $G$ is an open set with $G \subset B(0,1), \partial G$ is locally Lipschitz, and $H^{1}(\partial G \cap \partial B(0,1))>0$, then the harmonic measure of $\partial G \cap \partial B(0,1)$ with respect to some point in $G$ is positive. In our situation we show, following [L], that if $G \subset \Omega$ is an NTA-domain (see Definition 3.1), then

$$
\mu(\partial G \cap \partial \Omega)>0 \Rightarrow \omega(\partial G \cap \partial \Omega)>0 .
$$

Here $\omega$ is elliptic measure defined with respect to a point in $G$, and with respect to the operator $L$ in (2.7). To prove (2.10) we use results from [FJK1], [FJK2], [FKS] on elliptic PDE whose ellipticity is given in terms of an $A_{2}$-weight and argue as in [DJK].

In order to state Theorems 2-4 we next briefly describe the construction of Wolff snowflakes introduced in [W2]. Let $\Omega_{0}=\left\{\left(x^{\prime}, x_{n}\right): x^{\prime} \in \mathbb{R}^{n-1}, x_{n}>0\right\}$ and set $Q(r)=\left\{x^{\prime} \in \mathbb{R}^{n-1}:-r / 2 \leq x_{i} \leq r / 2\right.$ for $\left.1 \leq i \leq n-1\right\}$. Then $Q(r)$ is an $n-1-$ dimensional cube with sidelength $r$ and center at 0 . Let $\phi: \mathbb{R}^{n-1} \rightarrow \mathbb{R}$ be a piecewise linear function with supp $\phi \subset\left\{x^{\prime}:\left|x^{\prime}\right|<1 / 2\right\}$ and $\|\nabla \phi\|_{\infty} \leq M$. For fixed $N$ large, set $\psi\left(x^{\prime}\right)=N^{-1} \phi\left(N x^{\prime}\right)$. Let $b>0$ be a small constant and let $Q$ be an $n-1$-cube (i.e., an $n$-1-dimensional cube contained in some hyperplane) with center $a_{Q}$ and sidelength $l(Q)$. Let $e$ be a unit normal to $Q$ and define

$$
P_{Q}=\operatorname{cch}\left(Q \cup\left\{a_{Q}+b l(Q) e\right\}\right), \quad \tilde{P}_{Q}=\operatorname{int} \operatorname{cch}\left(Q \cup\left\{a_{Q}-b l(Q) e\right\}\right),
$$

where cch $E$ and int $E$ denote the closed convex hull and interior of $E$, respectively. For the cube $Q(1)$ set $e=-e_{n}$ and put

$\Lambda=\left\{x \in P_{Q(1)} \cup \tilde{P}_{Q(1)}: x_{n} \geq \psi(x)\right\}, \partial=\left\{x \in \mathbb{R}^{n}: x^{\prime} \in Q(1), x_{n}=\psi\left(x^{\prime}\right)\right\}$.

We assume that $N=N(b, M)$ is so large that $d\left(\partial \backslash \partial \Omega_{0}, \partial\left[P_{Q(1)} \cup \tilde{P}_{Q(1)}\right]\right) \geq b / 100$. Note that $\partial \subset Q(1) \times[-1 / 2,1 / 2]$ consists of a finite number of $n-1$-dimensional faces. We fix a Whitney decomposition of each face. That is, we divide each face of $\partial$ into $n-1$ cubes $Q$, with sidelengths $8^{-k}, k=1,2, \ldots$, which are proportional to their distance from the edges of the face they lie on. We also choose a distinguished $n-2$-dimensional 'side' for each $n-1$-cube.

Suppose $\Omega$ is a domain and $Q \subset \partial \Omega$ is an $n-1$-cube with distinguished side $\gamma$. Let $e$ be the outer unit normal to $\partial \Omega$ on $Q$ and suppose that $P_{Q} \cap \Omega=\emptyset$ and $\tilde{P}_{Q} \subset \Omega$. We form a new domain $\tilde{\Omega}$ as follows. Let $T$ be the conformal affine map (i.e., a composition of a translation, rotation, dilation) with $T(Q(1))=Q$ which fixes the dilation, $T(0)=a_{Q}$ which fixes the translation, and finally fix the rotation by requiring that $T(\{x \in \partial Q(1)$ : $\left.\left.x_{1}=1 / 2\right\}\right)=\gamma$ and $T\left(-e_{n}\right)$ is in the direction of $e$. Let $\Lambda_{Q}=T(\Lambda)$ and $\partial_{Q}=T(\partial)$. Then we define $\tilde{\Omega}$ through the relations $\tilde{\Omega} \cap\left(P_{Q} \cup \tilde{P}_{Q}\right)=\Lambda_{Q}$ and $\tilde{\Omega} \backslash\left(P_{Q} \cup \tilde{P}_{Q}\right)=$ 
$\Omega \backslash\left(P_{Q} \cup \tilde{P}_{Q}\right)$. Note that $\partial_{Q}$ inherits from $\partial$ a natural subdivision into Whitney cubes with distinguished sides. We call this process 'adding a blip to $\Omega$ along $Q$ '.

To use the process of 'adding a blip' to construct a Wolff snowflake $\Omega_{\infty}$, starting from $\Omega_{0}$, we first add a blip to $\Omega_{0}$ along $Q(1)$ obtaining a new domain $\Omega_{1}$. We then inherit a subdivision of $\partial \Omega_{1} \cap\left(P_{Q(1)} \cup \tilde{P}_{Q(1)}\right)$ into Whitney cubes with distinguished sides, together with a finite set of edges $E_{1}$ (the edges of the faces of the graph are not in the Whitney cubes). Let $G_{1}$ be the set of all Whitney cubes in the subdivision. Then $\Omega_{2}$ is obtained from $\Omega_{1}$ by adding a blip along each $Q \in G_{1}$. From this process, we inherit a family of cubes $G_{2} \subset \partial \Omega_{2}$ (each with a distinguished side) and a set of edges $E_{2} \subset \partial \Omega_{2}$ of $\sigma$-finite $H^{n-2}$-measure. Continuing by induction we get $\left(\Omega_{m}\right)_{n-1}^{\infty},\left(G_{m}\right)_{n-1}^{\infty},\left(E_{m}\right)_{n-1}^{\infty}$, where $\partial \Omega_{m} \cap\left(P_{Q(1)} \cup \tilde{P}_{Q(1)}\right)=E_{m} \cup \bigcup_{Q \in G_{m}} Q$ for $m \geq 1$. In [W2], Wolff shows, for $n=3$ and in a similar situation, that if $N=N(b, M)$ is large enough, then $\Omega_{m} \rightarrow \Omega_{\infty}$ in the Hausdorff distance sense. We call $\Omega_{\infty}$ a Wolff snowflake.

For fixed $p \in(1, \infty), p \neq 2$, let $u_{\infty}=u_{\infty}(\cdot, p)$ be the positive $p$-harmonic function in $\Omega_{\infty}$ with continuous boundary value zero on $\partial \Omega_{\infty}$ and $\left|x_{n}-u_{\infty}(x)\right| \rightarrow 0$ uniformly as $|x| \rightarrow \infty$. Let $\mu_{\infty}$ be the $p$-harmonic measure associated with $u_{\infty}$ and let $\mu_{\infty}^{\prime}$ be the restriction of $\mu_{\infty}$ to $(Q(1) \times[-1,1]) \cap \partial \Omega_{\infty}$. More details concerning the construction of $\Omega_{\infty}, u_{\infty}$ will be given in Section 6. In particular, in Lemma 6.1 we prove the existence and uniqueness of such a $u_{\infty}$.

Next we state our second main result.

Theorem 2. Let $\Omega_{\infty}, u_{\infty}, \mu_{\infty}$ be as above. If $p \geq n \geq 3$, then $\mathrm{H}-\operatorname{dim} \mu_{\infty}^{\prime}<n-1$. Moreover, if $2<p<n$ then there exists $\Omega_{\infty}$ such that $\mathrm{H}-\operatorname{dim} \mu_{\infty}^{\prime}<n-1$, while if $1<p<2$ then there exists $\Omega_{\infty}$ such that $\mathrm{H}-\operatorname{dim} \mu_{\infty}^{\prime}>n-1$.

Outline of the proof of Theorem 2. The proof of Theorem 2 follows after the proof of Proposition 7.6 and Theorem 3 in Section 7. As noted above, in [W2] Wolff constructed snowflakes for which the Hausdorff dimension of harmonic measure is either larger than $n-1$ or less than $n-1$. In his ingenious arguments, Wolff uses ideas of Carleson [C], boundary Harnack inequalities and estimates for the Green function from [D], [JK], as well as a high level of technical skill, in order to obtain his rather deep results. His proof depends heavily on the linearity of the Laplace operator and hence until recently it seemed unlikely that his program could be made to work for the nonlinear $p$-Laplacian when $1<p<\infty, p \neq 2$. Indeed, as mentioned above, only recently have the first and second authors in [LN1]-[LN6] and [LLuN] developed the basic tools for $p$-harmonic functions, $p \neq 2$, necessary to begin writing this paper. Besides (2.6), these tools include boundary Harnack inequalities for the ratio of two nonnegative $p$-harmonic functions, vanishing on a portion of a Lipschitz or a sufficiently flat Reifenberg flat domain (see Theorem 3.9). Another important tool is the generalization to the setting of $p$-harmonic measure (see [LN3]) of the classical result of Dahlberg [D] concerning the absolute continuity of harmonic measure with respect to surface measure (see Theorem 3.10 below). By using these recently developed tools the gist of Theorem 2 is that Wolff's program can also be made to work in the $p$-harmonic setting.

To outline our proof, let $\hat{\Omega}(\epsilon)=\left\{\left(x^{\prime}, x_{n}\right): x_{n}>\epsilon \hat{\theta}\left(x^{\prime}\right), x^{\prime} \in \mathbb{R}^{n-1}\right\}$. For fixed $p \in(1, \infty)$, let $\hat{u}=\hat{u}(\cdot, \epsilon)$ be the unique $p$-harmonic function in $\hat{\Omega}$ such that $\hat{u} \equiv 0$ on 
$\partial \hat{\Omega}$ and $\left|x_{n}-\hat{u}(x)\right| \rightarrow 0$ uniformly as $|x| \rightarrow \infty, x \in \hat{\Omega}$ (see Lemma 6.1). Furthermore, let

$$
I=I(\epsilon)=\int_{\partial \hat{\Omega}(\epsilon)}|\nabla \hat{u}(\cdot, \epsilon)|^{p-1} \log |\nabla \hat{u}(\cdot, \epsilon)| d H^{n-1} .
$$

In Section 6 we prove existence of $I(\epsilon)$ and its differentiability with respect to $\epsilon$, provided $\hat{\theta} \in C_{0}^{\infty}\left(\mathbb{R}^{n-1}\right)$. In fact in Lemma 6.3 we show that

$$
I(0)=0, \quad I^{\prime}(0)=0, \quad I^{\prime \prime}(0)=\frac{p-2}{p-1} \int_{\mathbb{R}^{n-1}}\left|\nabla^{\prime} \hat{\theta}\right|^{2} d H^{n-1},
$$

where $\nabla^{\prime}$ denotes the gradient in the variables $x^{\prime} \in \mathbb{R}^{n-1}$. From Taylor's theorem it follows that $I(\epsilon)>0$ for $p>2$ while $I(\epsilon)<0$ when $1<p<2$, provided $0<\epsilon \leq \epsilon_{0}$ and $\epsilon_{0}=\epsilon_{0}(p, n, \hat{\theta})$ is small enough. In case $p=2$ this calculation shows that $I^{\prime \prime}(0)=0$ and then one is forced to evaluate integrals in order to determine the sign for $I^{\prime \prime \prime}(0)$ or even $I^{(4)}(0)$, as is done in [W2] and [LVV]. The choice of $\hat{\theta}$ determines the sign and it seems remarkable that for $p \neq 2$ the sign depends on $p$ only and not on $\hat{\theta}$.

As the next step we then show (see Propositions $6.4,6.5$ ) that we can approximate $\epsilon \hat{\theta}$ by a piecewise linear function, $\phi=\phi(\cdot, \epsilon)$, in such a way that the sign of $I(\epsilon)$ is preserved. More specifically let $\tilde{\Omega}=\left\{x \in \mathbb{R}^{n}: x_{n}>\phi\left(x^{\prime}\right), x^{\prime} \in \mathbb{R}^{n-1}\right\}$ and let $\tilde{u}$ be the corresponding positive $p$-harmonic function in $\tilde{\Omega}$ with continuous boundary value 0 on $\partial \tilde{\Omega}$ and $\left|x_{n}-\tilde{u}(x)\right| \rightarrow 0$ uniformly as $|x| \rightarrow \infty$. We prove that

$$
\tilde{I}=\int_{\partial \tilde{\Omega}}|\nabla \tilde{u}|^{p-1} \log |\nabla \tilde{u}| d H^{n-1}
$$

has the same sign as $I(\epsilon)$. Next we construct a Wolff snowflake $\Omega_{\infty}$, as above, relative to $\psi\left(x^{\prime}\right)=N^{-1} \phi\left(x^{\prime} / N\right), x^{\prime} \in \mathbb{R}^{n-1}$, with $\Omega_{1}=\left\{\left(x^{\prime}, x_{n}\right): x_{n}>\psi\left(x^{\prime}\right), x^{\prime} \in \mathbb{R}^{n-1}\right\}$. Let $\Omega_{\infty}, u_{\infty}, \mu_{\infty}, \mu_{\infty}^{\prime}$ be the corresponding domain, $p$-harmonic function, measure, and restriction of the measure, as defined above Theorem 2 . We then prove the following theorem in Section 7.

Theorem 3. There exist $\theta_{0} \in(0,1)$ and $N_{0}$ large, depending on $p, n$, such that if $\|\nabla \phi\|_{\infty} \leq \theta_{0}, N \geq N_{0}$, and $\tilde{I}>0$, then $\mathrm{H}-\operatorname{dim} \mu_{\infty}^{\prime}<n-1$, while if $\tilde{I}<0$, then $\mathrm{H}-\operatorname{dim} \mu_{\infty}^{\prime}>n-1$.

From Theorem 3 and the above discussion we conclude that if $2<p<n$, then there exist Wolff snowflakes for which $\mathrm{H}$ - $\operatorname{dim} \mu_{\infty}^{\prime}<n-1$, while if $1<p<2$, then there exist Wolff snowflakes for which H-dim $\mu_{\infty}^{\prime}>n-1$. If $p \geq n$, then we can use the fact that $\log |\nabla \tilde{u}|$ is a subsolution to (2.7), with $u$ replaced by $\tilde{u}$, in order to show that $\tilde{I}>0$ for any Lipschitz function $\phi$ with compact support in $\left\{x^{\prime} \in \mathbb{R}^{n-1}:\left|x^{\prime}\right|<1 / 2\right\}$. Thus for $p \geq n$ we always get $\mathrm{H}-\operatorname{dim} \mu_{\infty}^{\prime}<n-1$ when using the Wolff method. This gives Theorem 2. Intuitively, the $p \geq n$ result says that adding any sort of Lipschitz blip to a half-space increases the ' $p$-entropy'.

Theorems 2 and 3 seem to indicate that the direct analogue of [BL] may hold in $\mathbb{R}^{n}$, $n \geq 3$. However, the situation is much more interesting, as we show in Theorem 4 . To avoid confusion, for fixed $p \in(1, \infty)$, we here write $\mu_{\infty}(\cdot, p), \mu_{\infty}^{\prime}(\cdot, p)$ for the above measures. 
Theorem 4. There is a Wolff snowflake for which $\mu_{\infty}^{\prime}(\cdot, p)$, for $p$ in an open interval containing 2 , and $\mu_{\infty}^{\prime}(\cdot, 2)$ both have $\mathrm{H}$-dim either $>n-1$ or $<n-1$.

In fact our proof will show that the results of [LVV] for interior and exterior harmonic measures in a Wolff snowflake remain valid for interior and exterior $p$-harmonic measures provided $p$ is close enough to 2 . The proof of Theorem 4 is similar to the proof of Theorem 3, except that now all estimates must be made independent of $p$ when $p \in[3 / 2,5 / 2]$. In particular, we prove that a smooth graph for which the corresponding $I$ is nonzero at $p=2$ can be approximated by piecewise linear graphs, for which the corresponding integrals, as functions of $p$, have the same sign in an open interval containing 2 (see Propositions 6.4, 6.5).

\section{Estimates for $p$-harmonic functions}

Definition 3.1. A domain $\Omega$ is called non-tangentially accessible (NTA) if there exist $M \geq 2$ and $r_{0}>0$ such that the following are fulfilled:

(i) corkscrew condition: for any $w \in \partial \Omega$ and $0<r<r_{0}$, there exists $a_{r}(w) \in \Omega$ satisfying $M^{-1} r<\left|a_{r}(w)-w\right|<r$ and $d\left(a_{r}(w), \partial \Omega\right)>M^{-1} r$

(ii) $\mathbb{R}^{n} \backslash \bar{\Omega}$ satisfies the corkscrew condition,

(iii) uniform condition: if $w \in \partial \Omega, 0<r<r_{0}$, and $w_{1}, w_{2} \in B(w, r) \cap \Omega$, then there exists a rectifiable curve $\gamma:[0,1] \rightarrow \Omega$ with $\gamma(0)=w_{1}$ and $\gamma(1)=w_{2}$ such that

(a) $H^{1}(\gamma) \leq M\left|w_{1}-w_{2}\right|$,

(b) $\min \left\{H^{1}(\gamma([0, t])), H^{1}(\gamma([t, 1]))\right\} \leq M d(\gamma(t), \partial \Omega)$.

We note that (iii) is different from but equivalent to the usual Harnack chain condition given in $[\mathrm{JK}]$ (see [BL, Lemma 2.5]). We choose this definition in order to emphasize the dependence of $\Omega$ on $M$. The constants $M, r_{0}$ will be called the NTA-constants of $\Omega$.

Let $1<p<\infty$ and let $\Omega \subset \mathbb{R}^{n}$ be a domain. Throughout this section and this paper, unless otherwise stated, $c$ will denote a positive constant $\geq 1$, not necessarily the same at each occurrence, depending only on $p, n, M$. In general, $c\left(a_{1}, \ldots, a_{m}\right)$ denotes a positive constant $\geq 1$, which may depend only on $p, n, M$ and $a_{1}, \ldots, a_{m}$, and which is not necessarily the same at each occurrence. If $A \approx B$ then $A / B$ is bounded from above and below by positive constants which, unless otherwise stated, only depend on $p, n, M$.

\subsection{Estimates in NTA-domains}

For the proof of Lemmas 3.2-3.6 we refer to [LN1]. In these lemmas, $\Delta(w, r)=$ $\partial \Omega \cap B(w, r)$.

Lemma 3.2. Given $p \in(1, \infty)$, let $u$ be a positive $p$-harmonic function in $B(w, 2 r)$. Then

$$
\begin{aligned}
& \text { (i) } r^{p-n} \int_{B(w, r / 2)}|\nabla u|^{p} d x \leq c\left(\max _{B(w, r)} u\right)^{p} \text {, } \\
& \text { (ii) } \max _{B(w, r)} u \leq c \min _{B(w, r)} u .
\end{aligned}
$$


Furthermore, there exists $\alpha=\alpha(p, n) \in(0,1)$ such that if $x, y \in B(w, r)$, then

$$
\text { (iii) }|u(x)-u(y)| \leq c(|x-y| / r)^{\alpha} \max _{B(w, 2 r)} u \text {. }
$$

Lemma 3.3. Let $\Omega \subset \mathbb{R}^{n}$ be an NTA-domain and suppose that $p \in(1, \infty)$ is given. Let $w \in \partial \Omega, 0<r<r_{0}$, and suppose that $u$ is a nonnegative continuous $p$-harmonic function in $\bar{\Omega} \cap B(w, 2 r)$ and that $u=0$ on $\Delta(w, 2 r)$. Then

$$
\text { (i) } r^{p-n} \int_{\Omega \cap B(w, r / 2)}|\nabla u|^{p} d x \leq c\left(\max _{\Omega \cap B(w, r)} u\right)^{p} .
$$

Furthermore, there exists $\alpha=\alpha(p, n, M) \in(0,1)$ such that if $x, y \in \Omega \cap B(w, r)$, then

$$
\text { (ii) }|u(x)-u(y)| \leq c(|x-y| / r)^{\alpha} \max _{\Omega \cap B(w, 2 r)} u .
$$

Lemma 3.4. Let $\Omega \subset \mathbb{R}^{n}$ be an NTA-domain and suppose that $p \in(1, \infty)$ is given. Let $w \in \partial \Omega, 0<r<r_{0}$, and suppose that $u$ is a non-negative continuous $p$-harmonic function in $\bar{\Omega} \cap B(w, 2 r)$ and that $u=0$ on $\Delta(w, 2 r)$. There exists $c=c(p, n, M) \in$ $[1, \infty)$ such that if $\tilde{r}=r / c$, then

$$
\max _{\Omega \cap B(w, \tilde{r})} u \leq c u\left(a_{\tilde{r}}(w)\right) .
$$

Lemma 3.5. Let $\Omega \subset \mathbb{R}^{n}$ be an NTA-domain and suppose that $p \in(1, \infty)$ is given. Let $w \in \partial \Omega, 0<r<r_{0}$, and suppose that $u$ is a non-negative continuous $p$-harmonic function in $\bar{\Omega} \cap B(w, 4 r)$ and $u=0$ on $\Delta(w, 4 r)$. Extend $u$ to $B(w, 4 r)$ by defining $u \equiv 0$ on $B(w, 4 r) \backslash \Omega$. Then $u$ has a representative in $W^{1, p}(B(w, 4 r))$ with Hölder continuous partial derivatives in $\Omega \cap B(w, 4 r)$. In particular, there exists $\sigma \in(0,1]$, depending only on $p, n$, such that if $x, y \in B(\hat{w}, \hat{r} / 2)$, where $B(\hat{w}, 4 \hat{r}) \subset \Omega \cap B(w, 4 r)$, then

$$
c^{-1}|\nabla u(x)-\nabla u(y)| \leq(|x-y| / \hat{r})^{\sigma} \max _{B(\hat{w}, \hat{r})}|\nabla u| \leq c \hat{r}^{-1}(|x-y| / \hat{r})^{\sigma} \max _{B(\hat{w}, 2 \hat{r})} u .
$$

If $\nabla u(\hat{w}) \neq 0$, then $u$ is real analytic in a neighborhood of $\hat{w}$.

Lemma 3.6. Let $\Omega \subset \mathbb{R}^{n}$ be an NTA-domain. Given $p \in(1, \infty), w \in \partial \Omega, 0<$ $r<r_{0}$, suppose that $u$ is a positive p-harmonic function in $\Omega \cap B(w, 2 r)$, continuous in $\bar{\Omega} \cap B(w, 2 r)$, with $u=0$ on $\Delta(w, 2 r)$. Extend $u$ to $B(w, 2 r)$ by defining $u \equiv 0$ on $B(w, 2 r) \backslash \Omega$. There exists a unique locally finite positive Borel measure $\mu$ on $\mathbb{R}^{n}$, with support in $\Delta(w, 2 r)$, such that for all $\theta \in C_{0}^{\infty}(B(w, 2 r))$,

$$
\text { (i) } \int|\nabla u|^{p-2}\langle\nabla u, \nabla \theta\rangle d x=-\int \theta d \mu \text {. }
$$

Moreover, there exists $c=c(p, n, M) \in[1, \infty)$, such that if $\tilde{r}=r / c$, then

(ii) $\quad c^{-1} r^{p-n} \mu(\Delta(w, \tilde{r})) \leq\left(u\left(a_{\tilde{r}}(w)\right)\right)^{p-1} \leq c r^{p-n} \mu(\Delta(w, \tilde{r} / 2))$. 


\subsection{Estimates in Reifenberg flat domains}

Theorem 3.7 ([LN5, Lemma 3.8]). Let $\Omega \subset \mathbb{R}^{n}$ be a $\left(\delta, r_{0}\right)$-Reifenberg flat domain. Given $p \in(1, \infty), w \in \partial \Omega$, and $0<r<r_{0}$, suppose that $u$ is a positive $p$-harmonic function in $\Omega \cap B(w, 4 r)$, $u$ is continuous in $\bar{\Omega} \cap B(w, 4 r)$, and $u=0$ on $\Delta(w, 4 r)$. Then there exist $\bar{\delta}_{1}=\bar{\delta}_{1}(p, n)>0, \bar{c}_{1}=\bar{c}_{1}(p, n) \in[1, \infty)$ and $\bar{\lambda}=\bar{\lambda}(p, n) \in[1, \infty)$ such that if $0<\delta<\bar{\delta}_{1}$, then

$$
\bar{\lambda}^{-1} \frac{u(y)}{d(y, \partial \Omega)} \leq|\nabla u(y)| \leq \bar{\lambda} \frac{u(y)}{d(y, \partial \Omega)} \quad \text { whenever } y \in \Omega \cap B\left(w, r / \bar{c}_{1}\right) .
$$

Lemma 3.8 ([LN5, Lemma 3.15]). Let $\Omega \subset \mathbb{R}^{n}$ be a $\left(\delta, r_{0}\right)$-Reifenberg flat domain. Given $p \in(1, \infty), w \in \partial \Omega$, and $0<r<r_{0}$, suppose that $u$ is a positive $p$-harmonic function in $\Omega \cap B(w, 4 r)$, $u$ is continuous in $\bar{\Omega} \cap B(w, 4 r)$, and $u=0$ on $\Delta(w, 4 r)$. Then there exist, for $\epsilon>0$ given, $\bar{\delta}_{2}=\bar{\delta}_{2}(p, n, \epsilon)>0$ and $\bar{c}_{2}=\bar{c}_{2}(p, n, \epsilon) \in[1, \infty)$ such that

$$
\bar{c}_{2}^{-1}\left(\frac{\hat{r}}{r}\right)^{1+\epsilon} \leq \frac{u\left(a_{\hat{r}}(w)\right)}{u\left(a_{r}(w)\right)} \leq \bar{c}_{2}\left(\frac{\hat{r}}{r}\right)^{1-\epsilon} \quad \text { whenever } 0<\delta<\bar{\delta}_{2} \text { and } 0<\hat{r}<r / 4
$$

Theorem 3.9 ([LN5, Theorem 1]). Let $\Omega \subset \mathbb{R}^{n}$ be a $\left(\delta, r_{0}\right)$-Reifenberg flat domain. Given $p \in(1, \infty), w \in \partial \Omega$, and $0<r<r_{0}$, suppose that $u$, $v$ are positive $p$-harmonic functions in $\Omega \cap B(w, 4 r), u, v$ are continuous in $\bar{\Omega} \cap B(w, 4 r)$, and $u=0=v$ on $\Delta(w, 4 r)$. Then there exist $\bar{\delta}_{3}=\bar{\delta}_{3}(p, n)>0, \bar{c}_{3}=\bar{c}_{3}(p, n) \in[1, \infty)$, and $\bar{\sigma}=$ $\bar{\sigma}(p, n) \in(0,1)$ such that if $0<\delta<\bar{\delta}_{3}$, then

$$
\left|\log \frac{u\left(y_{1}\right)}{v\left(y_{1}\right)}-\log \frac{u\left(y_{2}\right)}{v\left(y_{2}\right)}\right| \leq \bar{c}_{3}\left(\frac{\left|y_{1}-y_{2}\right|}{r}\right)^{\bar{\sigma}} \quad \text { whenever } y_{1}, y_{2} \in \Omega \cap B\left(w, r / \bar{c}_{3}\right) .
$$

\subsection{Estimates in Lipschitz domains}

Let $\Omega \subset \mathbb{R}^{n}$ be an NTA-domain. We say that $\Omega$ is a Lipschitz domain on scale $t, t \ll r_{0}$, with Lipschitz constant $M^{\prime}$ if for all $w \in \partial \Omega$ and $0<r<t$ there exists a Lipschitz function $\phi$, with Lipschitz constant bounded by $M^{\prime}$, such that

$$
\begin{aligned}
\Omega \cap B(w, 4 r) & =\left\{y=\left(y^{\prime}, y_{n}\right) \in \mathbb{R}^{n}: y_{n}>\phi\left(y^{\prime}\right)\right\} \cap B(w, 4 r), \\
\partial \Omega \cap B(w, 4 r) & =\left\{y=\left(y^{\prime}, y_{n}\right) \in \mathbb{R}^{n}: y_{n}=\phi\left(y^{\prime}\right)\right\} \cap B(w, 4 r),
\end{aligned}
$$

in an appropriate coordinate system. We let $e_{i}, 1 \leq i \leq n$, denote the point in $\mathbb{R}^{n}$ with 1 in the $i$ th coordinate position and zeroes elsewhere. In the following we will assume that, in addition, $\Omega \subset \mathbb{R}^{n}$ is a $\left(\delta, r_{0}\right)$-Reifenberg flat domain with $\delta<\hat{\delta}(n)$. Hence $M^{\prime} \approx \delta$. If $\Omega$ is a Lipschitz domain on scale $t$, and $t \ll r_{0}, w \in \partial \Omega$, then for $0<b<1$ and $y \in \partial \Omega$ we let

$$
\Gamma(y)=\Gamma_{b}(y)=\{x \in \Omega: d(x, \partial \Omega)>b|x-y|\} .
$$


Given $w \in \partial \Omega, 0<r<t$, and a measurable function $k$ on $\bigcup_{y \in \Delta(w, 2 r)} \Gamma(y) \cap B(w, 4 r)$ we define the nontangential maximal function $N(k): \Delta(w, 2 r) \rightarrow \mathbb{R}$ for $k$ as

$$
N(k)(y)=\sup _{x \in \Gamma(y) \cap B(w, 4 r)}|k|(x) \quad \text { whenever } y \in \Delta(w, 2 r) .
$$

We let $L^{q}(\Delta(w, 2 r)), 1 \leq q \leq \infty$, be the space of functions which are integrable, with respect to $H^{n-1}$, to the power $q$ on $\Delta(w, 2 r)$. Let $\|f\|_{L^{q}(\Delta(w, 2 r))}$ be the norm of $f \in L^{q}(\Delta(w, 2 r))$. Furthermore, given a measurable function $f$ on $\Delta(w, 2 r)$ we say that $f$ is of bounded mean oscillation on $\Delta(w, r), f \in \operatorname{BMO}(\Delta(w, r))$, if there exists $A \in(0, \infty)$ such that

$$
\int_{\Delta(y, s)}\left|f-f_{\Delta}\right|^{2} d H^{n-1} \leq A^{2} H^{n-1}(\Delta(y, s))
$$

whenever $y \in \Delta(w, r)$ and $0<s \leq r$. Here $f_{\Delta}$ denotes the average of $f$ on $\Delta=\Delta(y, s)$ with respect to $H^{n-1}$. The least $A$ for which (3.4) holds is denoted by $\|f\|_{\operatorname{BMO}(\Delta(w, r))}$. If $f$ is a vector valued function, $f=\left(f_{1}, \ldots, f_{n}\right)$, then $f_{\Delta}=\left(f_{1, \Delta}, \ldots, f_{n, \Delta}\right)$ and the BMO-norm of $f$ is defined as in (3.4) with $\left|f-f_{\Delta}\right|^{2}=\left\langle f-f_{\Delta}, f-f_{\Delta}\right\rangle$. For more on $\mathrm{BMO}$ we refer the reader to [S, Chapter IV].

Theorem 3.10 ([LN3, Theorem 1]). Let $\Omega \subset \mathbb{R}^{n}$ be a $\left(\delta, r_{0}\right)$-Reifenberg flat domain with $\delta<\hat{\delta}(n)$ and assume, in addition, that $\Omega$ is a Lipschitz domain on scale $t, t \ll r_{0}$. Let $w \in \partial \Omega$ and $0<r<t$. Given $p \in(1, \infty)$, suppose that $u$ is a positive $p$-harmonic function in $\Omega \cap B(w, 4 r)$, $u$ is continuous in $\bar{\Omega} \cap B(w, 4 r)$, and $u=0$ on $\Delta(w, 4 r)$. Extend $u$ to $B(w, 4 r)$ by putting $u \equiv 0$ on $B(w, 4 r) \backslash \Omega$ and let $\mu$ be the associated measure corresponding to $u$ as in Lemma 3.6 with $2 r$ replaced by $4 r$. Then $\mu$ is absolutely continuous with respect to $H^{n-1}$ on $\Delta(w, 4 r)$. Moreover,

$$
\lim _{x \in \Gamma(y), x \rightarrow y} \nabla u(x)=\nabla u(y)
$$

for $H^{n-1}$-almost every $y \in \Delta(w, 4 r)$ and there exist $q>p$ and a constant $c \in[1, \infty)$, which both depend only on $p, n$, such that

(i) $\|N(\nabla u \mid)\|_{L^{q}(\Delta(w, 2 r))} \leq c r^{-1+(n-1) / q} u\left(a_{r}(w)\right)$,

(ii) $\int_{\Delta(w, 2 r)}|\nabla u|^{q} d H^{n-1} \leq c r^{(n-1) \frac{p-1-q}{p-1}}\left(\int_{\Delta(w, 2 r)}|\nabla u|^{p-1} d H^{n-1}\right)^{q /(p-1)}$,

(iii) $\log |\nabla u| \in \operatorname{BMO}(\Delta(w, r))$ and $\|\log |\nabla u|\|_{\operatorname{BMO}(\Delta(w, r))} \leq c$,

(iv) $\quad d \mu(x)=|\nabla u|^{p-1}(x) d H^{n-1}$ and $\nabla u(x) /|\nabla u(x)|$ is the inner unit normal to $\Omega \cap B(w, 2 r)$ for $H^{n-1}$-almost every $x$ in $\Delta(w, 2 r)$.

Note that using Theorem 3.10 we can conclude that

$$
\int_{\Delta(w, r)}|\nabla u|^{p} d H^{n-1} \leq c H^{n-1}(\Delta(w, r))\left(\frac{\mu(\Delta(w, r))}{H^{n-1}(\Delta(w, r))}\right)^{p /(p-1)}
$$


and hence

$$
\frac{1}{\mu(\Delta(w, r))} \int_{\Delta(w, r)} \eta^{-1}|\nabla u|^{p} d H^{n-1} \leq c \eta^{-1}\left(\frac{\mu(\Delta(w, r))}{H^{n-1}(\Delta(w, r))}\right)^{1 /(p-1)}
$$

whenever $\eta>0$. Next, using Jensen's inequality we therefore see that

$$
\begin{aligned}
\frac{1}{\mu(\Delta(w, r))} \int_{\Delta(w, r)}|\nabla u|^{p-1} \log \left(\eta^{-1}|\nabla u|\right) d H^{n-1} & \\
\leq & \log \left(c \eta^{-1}\left(\frac{\mu(\Delta(w, r))}{H^{n-1}(\Delta(w, r))}\right)^{1 /(p-1)}\right)
\end{aligned}
$$

whenever $\eta>0$. From (3.7), and boundedness of $x|\log x|$ on (0,1], we conclude that

$$
\begin{aligned}
\int_{\Delta(w, r)}|\nabla u|^{p-1}\left|\log \left(\eta^{-1}|\nabla u|\right)\right| d H^{n-1} & \leq \mu \mu(\Delta(w, r)) \\
& \text { if } \eta \approx\left(\frac{\mu(\Delta(w, r))}{H^{n-1}(\Delta(w, r))}\right)^{1 /(p-1)} .
\end{aligned}
$$

Remark 3.11. For use in the proof of Theorem 4 we note that the constants in Lemmas 3.2-3.6, 3.8 and Theorems 3.7, 3.9, 3.10 can be chosen independent of $p$ when $p \in[3 / 2,5 / 2]$. This statement can be proved by a straightforward but rather laborious checking of details, in the references listed for these results.

\section{Degenerate elliptic equations}

In this section we state some lemmas for degenerate elliptic operators in the sense of [FKS], [FJK1] and [FJK2]. They are the basis for many of our estimates in the proofs of Theorems 1-4. Let $w \in \mathbb{R}^{n}, r>0$, and let $\lambda(y)$ be a real valued Lebesgue measurable function defined almost everywhere on $B(w, 2 r)$. It is said to belong to the class $A_{2}(B(w, r))$ if there exists a constant $\gamma$ such that

$$
r^{-2 n} \int_{B(\tilde{w}, \tilde{r})} \lambda d y \cdot \int_{B(\tilde{w}, \tilde{r})} \lambda^{-1} d y \leq \gamma
$$

whenever $\tilde{w} \in B(w, r)$ and $0<\tilde{r} \leq r$. If $\lambda(y)$ belongs to the class $A_{2}(B(w, r))$ then $\lambda$ is referred to as an $A_{2}(B(w, r))$-weight. The smallest $\gamma$ such that (4.1) holds is referred to as the constant of the weight.

In the following we let $\Omega \subset \mathbb{R}^{n}$ be an NTA-domain with NTA-constants $M, r_{0}$. We let $w \in \partial \Omega, 0<r<r_{0}$, and we consider the operator

$$
\hat{L}=\sum_{i, j=1}^{n} \frac{\partial}{\partial y_{i}}\left(\hat{b}_{i j}(y) \frac{\partial}{\partial y_{j}}\right)
$$


in $\Omega \cap B(w, 2 r)$. We assume that the coefficients $\left\{\hat{b}_{i j}(y)\right\}$ are bounded, Lebesgue measurable functions defined almost everywhere on $B(w, 2 r)$. Moreover, $\hat{b}_{i j}=\hat{b}_{j i}$ for all $i, j \in\{1, \ldots, n\}$, and

$$
\beta^{-1} \lambda(y)|\xi|^{2} \leq \sum_{i, j=1}^{n} \hat{b}_{i j}(y) \xi_{i} \xi_{j} \leq \beta|\xi|^{2} \lambda(y)
$$

for almost every $y \in B(w, 2 r)$, where $\lambda \in A_{2}(B(w, r))$. By definition $\hat{L}$ is a degenerate elliptic operator (in divergence form) in $B(w, 2 r)$ with ellipticity measured by the function $\lambda$ and $\beta$. If $O \subset B(w, 2 r)$ is open then we let $\tilde{W}^{1,2}(O)$ be the weighted Sobolev space of equivalence classes of functions $v$ with distributional gradient $\nabla v$ and norm

$$
\| v \tilde{\|}_{1,2}^{2}=\int_{O} v^{2} \lambda d y+\int_{O}|\nabla v|^{2} \lambda d y<\infty
$$

Let $\tilde{W}_{0}^{1,2}(O)$ be the closure of $C_{0}^{\infty}(O)$ in the $\tilde{W}^{1,2}(O)$ norm. We say that $v$ is a weak solution to $\hat{L} v=0$ in $O$ provided $v \in \tilde{W}^{1,2}(O)$ and

$$
\int_{O} \sum_{i, j} \hat{b}_{i j} v_{y_{i}} \phi_{y_{j}} d y=0
$$

whenever $\phi \in C_{0}^{\infty}(O)$.

The following lemmas are based on the results in [FKS], [FJK1] and [FJK2]. In these lemmas $c$ depends on $n, M, \beta, \gamma$. Also $A \approx B$ means the ratio of $A / B$ is bounded above and below by constants having the same dependence as $c$.

Lemma 4.1. Let $\Omega \subset \mathbb{R}^{n}$ be an NTA-domain with constants $M, r_{0}, w \in \partial \Omega, 0<r<r_{0}$, and let $\lambda$ be an $A_{2}(B(w, r))$-weight with constant $\gamma$. Suppose that $v$ is a positive weak solution to $\hat{L} v=0$ in $\Omega \cap B(w, 2 r)$. Then there exists a constant $c \geq 1$ such that if $\tilde{w} \in \Omega, \tilde{r}>0$, and $B(\tilde{w}, 2 \tilde{r}) \subset \Omega \cap B(w, r)$, then

(i) $c^{-1} \tilde{r}^{2} \int_{B(\tilde{w}, \tilde{r} / 2)}|\nabla v|^{2} \lambda d y \leq c\left(\int_{B(\tilde{w}, \tilde{r})} \lambda d y\right)\left(\max _{B(\tilde{w}, \tilde{r})} v\right)^{2} \leq c \int_{B(\tilde{w}, 2 \tilde{r})}|v|^{2} \lambda d y$,

(ii) $\max _{B(\tilde{w}, \tilde{r})} v \leq c \min _{B(\tilde{w}, \tilde{r})} v$.

Furthermore, there exists $\alpha=\alpha(n, M, \beta, \gamma) \in(0,1)$ such that if $x, y \in B(\tilde{w}, \tilde{r})$ then

(iii) $|v(x)-v(y)| \leq c(|x-y| / \tilde{r})^{\alpha} \max _{B(\tilde{w}, 2 \tilde{r})} v$.

Lemma 4.2. Let $\Omega \subset \mathbb{R}^{n}$ be an NTA-domain with constants $M, r_{0}, w \in \partial \Omega, 0<r<r_{0}$, and let $\lambda$ be an $A_{2}(B(w, r))$-weight with constant $\gamma$. Suppose that $v$ is a positive weak 
solution to $\hat{L} v=0$ in $\Omega \cap B(w, 2 r)$ and that $v=0$ on $\Delta(w, 2 r)$ in the weighted Sobolev sense. Then there exists $1 \leq c<\infty$ such that the following holds with $\tilde{r}=r / c$ :

$$
\begin{aligned}
& \text { (i) } r^{2} \int_{\Omega \cap B(w, r / 2)}|\nabla v|^{2} \lambda d y \leq c \int_{\Omega \cap B(w, r)}|v|^{2} \lambda d y, \\
& \text { (ii) } \max _{\Omega \cap B(w, \tilde{r})} v \leq c v\left(a_{\tilde{r}}(w)\right) .
\end{aligned}
$$

Furthermore, there exists $\alpha=\alpha(n, M, \beta, \gamma) \in(0,1)$ such that if $x, y \in \Omega \cap B(w, \tilde{r})$, then

$$
\text { (iii) }|v(x)-v(y)| \leq c(|x-y| / r)^{\alpha} \max _{\Omega \cap B(w, 2 \tilde{r})} v .
$$

Proceeding as in [FJK1] and [FJK2] we can derive the existence of the Green function, $\hat{g}(\cdot, \cdot)$, associated to the operator $\hat{L}$. In particular, the following lemma can be proved.

Lemma 4.3. Let $\Omega \subset \mathbb{R}^{n}$ be an NTA-domain with constants $M, r_{0}$, and let $\lambda$ be an $A_{2}\left(\mathbb{R}^{n}\right)$-weight with constant $\gamma$. Then there exists a Green function $g(\cdot, \cdot): \Omega \times \Omega \rightarrow$ $(0, \infty]$, associated to the operator $\hat{L}$ with $\hat{L} g(\cdot, y)=0$ in $\Omega \backslash B(y, \epsilon)$ for each $y \in \hat{\Omega}$, $\epsilon>0$. The Green function also satisfies $g(x, y)=g(y, x)$ for $x \neq y$, and $\zeta(\cdot) g(\cdot, y) \in$ $\tilde{W}_{0}^{1,2}[\Omega \backslash \bar{B}(y, \epsilon)]$ whenever $\bar{B}(y, \epsilon) \subset \Omega$ and $\zeta \in C_{0}^{\infty}\left(\mathbb{R}^{n} \backslash \bar{B}(y, \epsilon)\right)$. For $y \in \Omega$ let $g(\cdot, y) \equiv 0$ on $\mathbb{R}^{n} \backslash \Omega$. Then $g(\cdot, y)$ is continuous on $\mathbb{R}^{n} \backslash\{y\}$. Finally, if $x \neq y$ and $d(y, \partial \Omega)<r_{0} / 4$, then for $n \geq 3$,

(a) $g(x, y) \approx \lambda(y)^{-1}|x-y|^{2-n}$ whenever $x \in B(y, d(y, \partial \Omega) / 2)$,

(b) $g(x, y) \leq c \lambda(y)^{-1}|x-y|^{2-n-\sigma} d(x, \partial \Omega)^{\sigma}$ for some $\sigma=\sigma(n, M, \gamma, \beta)$ whenever $x \in \Omega \backslash B(y, d(y, \partial \Omega) / 2)$.

Applying Lemmas 4.1 and 4.2 in $\Omega$ to $v(\cdot)=g(\cdot, y), y \in \Omega$, one can deduce the existence of a measure $\omega(\cdot, y)$, with support on $\partial \Omega, \omega(\partial \Omega, y)=1$, such that

$$
\theta(y)=\int \sum_{i, j=1}^{n} \theta_{x_{i}} \hat{b}_{i j} g_{x_{j}}(\cdot, y) d x+\int \theta d \omega(\cdot, y)
$$

whenever $\theta \in C_{0}^{\infty}\left(\mathbb{R}^{n}\right)$. We say that $\omega(\cdot, y)$ is the elliptic measure for $\Omega$ corresponding to $y$ and $\hat{L}$.

Our next step is to analyze the elliptic measure $\omega(\cdot, y)$. To do this we first note that using Lemmas 4.1-4.3 and (4.6) the following lemma can be derived (see [FJK1], [FJK2], for details).

Lemma 4.4. Let $\Omega \subset \mathbb{R}^{n}$ be an NTA-domain with constants $M, r_{0}$, and let $\lambda$ be an $A_{2}\left(\mathbb{R}^{n}\right)$-weight with constant $\gamma$. There exists $1 \leq c<\infty$ such that if $z \in \partial \Omega, 0<\rho<$ $r_{0} / c$, and $y \in \Omega \backslash B(z, 4 \rho)$, then

$$
c^{-1} \rho^{n-2} g\left(y, a_{\rho}(z)\right) \lambda\left(a_{\rho}(z)\right) \leq \omega(\partial \Omega \cap B(z, \rho), y) \leq c \rho^{n-2} g\left(y, a_{\rho}(z)\right) \lambda\left(a_{\rho}(z)\right) .
$$


We observe from Lemma 4.4 and Harnack's inequality that $\omega(\cdot, y)$ is a doubling measure in the following sense. There exists $1 \leq c<\infty$ such that

$$
\omega(B(x, 2 s), y) \leq c \omega(B(x, s), y) \quad \text { whenever } x \in \partial \Omega \cap B(z, 4 \rho), s \leq \rho .
$$

Moreover, using Lemma 4.3, Lemma 4.4 and Harnack's inequality we see that there exists a constant $c \geq 1$ such that if $z$ and $\rho$ are as in Lemma 4.4, then

$$
c \omega\left(\partial \Omega \cap B(z, \rho), a_{\rho / 2}(z)\right) \geq 1
$$

Let $\Omega, \lambda$ be as in Lemma 4.3. Using Lemma 4.2 and (4.7)-(4.8), it is not difficult to show that the continuous Dirichlet problem for $\Omega$ and $\hat{L}$ always has a solution. That is, given a continuous function $\theta$ on $\partial \Omega$ with compact support, there exists a weak solution $\Theta$ to $\hat{L}$ in $\Omega$ that takes on the boundary values $\theta$ continuously. In fact,

$$
\Theta(x)=\int \theta d \hat{\omega}(x, \cdot) \quad \text { whenever } x \in \Omega
$$

Next we state the main lemma of [DJK].

Lemma 4.5. Let $\Omega, \lambda, \hat{L}, \omega$ be as in Lemma 4.3 , and let $\tilde{\Omega} \subset \Omega$ be an NTA-domain with constants $M, r_{0}$. Let $\tilde{\omega}$ be elliptic measure for $\tilde{\Omega}$ corresponding to $\hat{L}$. Then there exist $c \geq 1$ and $\sigma>0$, depending on $M, n, \gamma$, such that the following is true. If $0<r \leq$ $r_{0} / c, w \in \partial \tilde{\Omega}$, and $E$ is a Borel subset of $\partial \Omega \cap \partial \tilde{\Omega} \cap B(w, r)$, then

$$
\omega\left(E, \tilde{a}_{r}(w)\right) \leq c \tilde{\omega}\left(E, \tilde{a}_{r}(w)\right)^{\sigma}
$$

In Lemma $4.5, \tilde{a}_{r}(w)$ is defined relative to $\tilde{\Omega}$ as in Definition 3.1. Observe that the inequality $\tilde{\omega}\left(E, \tilde{a}_{r}(w)\right) \leq \omega\left(E, \tilde{a}_{r}(w)\right)$ follows from the weak maximum principle for $\hat{L}$. We note that Lemma 4.5 is proved in detail only for uniformly elliptic operators and when $\tilde{\Omega}, \Omega$ are Lipschitz domains. However this lemma also remains valid in our situation (see the last section in [DJK]). For our purposes, it is enough to know that Lemma 4.5 is true when $E$ is 'most' of $\partial \hat{\Omega} \cap B(w, r)$, which is easier to prove. In fact a self-contained proof of (a) in Theorem B, readily adaptable to the current situation, can be found in [L].

Next we state a boundary Harnack inequality for positive solutions to $\hat{L}$ vanishing on a portion of $\partial \Omega$.

Lemma 4.6. Let $\Omega \subset \mathbb{R}^{n}$ be an NTA-domain with constants $M, r_{0}$, and let $\lambda$ be an $A_{2}\left(\mathbb{R}^{n}\right)$-weight with constant $\gamma$. Suppose that $w \in \partial \Omega, 0<r<r_{0} / 4$, and $v_{1}, v_{2}$ are two positive weak solutions to $\hat{L} v=0$ in $\Omega \cap B(w, 2 r)$ with $v_{1}=0=v_{2}$ on $\Delta(w, 2 r)$ in the weighted Sobolev sense. Then there exist $1 \leq c<\infty$ and $\alpha=\alpha(n, M, \beta, \gamma) \in(0,1)$ such that

$$
\left|\log \frac{v_{1}\left(y_{1}\right)}{v_{2}\left(y_{1}\right)}-\log \frac{v_{1}\left(y_{2}\right)}{v_{2}\left(y_{2}\right)}\right| \leq c\left(\frac{\left|y_{1}-y_{2}\right|}{r}\right)^{\alpha} \quad \text { whenever } y_{1}, y_{2} \in \Omega \cap B(w, r / c) .
$$


Finally in this section we point out our applications of the above lemmas to $p$-harmonic functions. Let $0<\delta<\hat{\delta}(n)$ and let $\Omega \subset \mathbb{R}^{n}$ be a $\left(\delta, r_{0}\right)$-Reifenberg flat domain. Given $p \in(1, \infty), w \in \partial \Omega$, and $0<r<r_{0}$, suppose that $u$ is a positive $p$-harmonic function in $\Omega \cap B(w, 4 r), u$ is continuous in $\bar{\Omega} \cap B(w, 4 r)$, and $u=0$ on $\Delta(w, 4 r)$. Using Theorem 3.7 we see that there exist $\bar{\delta}=\bar{\delta}_{1}(p, n)>0, \bar{c}_{1}=\bar{c}_{1}(p, n)$, and $\tilde{a}=\tilde{a}(p, n)$ such that if $0<\delta<\bar{\delta}_{1}$, then

$$
\tilde{a}^{-1} \frac{u(y)}{d(y, \partial \Omega)} \leq|\nabla u(y)| \leq \tilde{a} \frac{u(y)}{d(y, \partial \Omega)}
$$

whenever $y \in \Omega \cap B\left(w, r / \bar{c}_{1}\right)$. We refer to (4.10) as the fundamental inequality for $u$ in $\Omega \cap B\left(w, r / \bar{c}_{1}\right)$. Using Theorem 3.7 and Lemma 3.8, the following can be proved.

Lemma 4.7 ([LN5, Lemma 3.30]). Let $\Omega$ be a $\left(\delta, r_{0}\right)$-Reifenberg flat domain, $w \in \partial \Omega$, and $0<r<r_{0} / 4$. Fix $p \in(1, \infty)$, and suppose that $u$ is a positive $p$-harmonic function in $\Omega \cap B(w, 4 r)$, $u$ is continuous in $\bar{\Omega} \cap B(w, 4 r)$, and $u=0$ on $\Delta(w, 4 r)$. Then there exist $\delta_{4}=\delta_{4}(p, n)>0$ and $\bar{c}_{4}=\bar{c}_{4}(p, n) \geq 1$ such that if $0<\delta<\bar{\delta}_{4}$ and $\hat{r}=r / \bar{c}_{4}$, then $|\nabla u|^{p-2}$ extends to an $A_{2}(B(w, 2 \hat{r}))$-weight with constant depending only on $p$ and $n$. Moreover, the above constants can be chosen independent of $p$ when $p \in[3 / 2,5 / 2]$.

As an application of Lemma 4.7 suppose $r_{0}=\infty$ and let $\hat{b}_{i j}(y)=b_{i j}(y)$ whenever $y \in \Omega$, where $b_{i j}(y), 1 \leq i, j \leq n$, are defined in (2.8). From (2.7), (2.9) we see that the lemmas in this section can be applied to solutions of $L$. Observe from (4.10) that if $y \in \Omega$, then $\lambda(y) \approx(u(y) / d(y, \partial \Omega))^{p-2}$, where for $\delta>0$ small enough, the constants in the ratio depend only on $p, n$, and can be chosen independent of $p$ when $p \in[3 / 2,5 / 2]$.

\section{Proof of Theorem 1}

To begin the proof of Theorem 1 recall that we are assuming $\Omega$ is a $\delta$-Reifenberg flat domain with $r_{0}=\infty$, where $\delta$ is so small that Theorems 3.9 and 3.7 hold. Letting $r \rightarrow \infty$ in Theorem 3.9 we see that any two $p$-harmonic functions under consideration are constant multiples of each other. Moreover, using Lemmas 3.2-3.6 and compactness arguments, it is easily shown that there exists such a $p$-harmonic function $u$ with $u \not \equiv 0$. Using Theorem 3.7 and Lemma 3.5 we see that the function $u$ is infinitely differentiable and that $|\nabla u| \neq 0$ in $\Omega$. Recall from (2.7) and (2.8) that $u, u_{x_{k}}, 1 \leq k \leq n$, are solutions to

$$
L \zeta(x)=\sum_{i, j=1}^{n} \frac{\partial}{\partial x_{i}}\left[b_{i j}(x) \zeta_{x_{j}}(x)\right]=0
$$

where, at $x \in \Omega$,

$$
b_{i j}(x)=|\nabla u|^{p-4}\left[(p-2) u_{x_{i}} u_{x_{j}}+\delta_{i j}|\nabla u|^{2}\right](x), \quad 1 \leq i, j \leq n,
$$

and $\delta_{i j}$ is the Kronecker $\delta$. Also at $x \in \Omega$,

$$
\min \{p-1,1\}|\xi|^{2}|\nabla u(x)|^{p-2} \leq \sum_{i, j=1}^{n} b_{i j} \xi_{i} \xi_{j} \leq \max \{1, p-1\}|\nabla u(x)|^{p-2}|\xi|^{2} .
$$


From (5.3) and Lemma 4.7 we deduce that

$$
|\nabla u|^{p-2} \text { extends to an } A_{2} \text {-weight on } \mathbb{R}^{n} .
$$

To prove Theorem 1 we will need the following lemma.

Lemma 5.1. Let $\tau(x)=\log |\nabla u(x)|^{2}$ whenever $x \in \Omega$. If $p \geq n$, then L $\tau \geq 0$ in $\Omega$.

Proof. For fixed $a \in \Omega$ we assume, as we may since (5.1) is invariant under rotations, that $\nabla u(a)=u_{x_{1}}(a) e_{1}$. Let $\tau=\log |\nabla u|^{2}$ and note that

$$
\tau_{x_{j}}=\sum_{k=1}^{n} \frac{2 u_{x_{k}} u_{x_{k} x_{j}}}{|\nabla u|^{2}}
$$

Furthermore,

$$
L \tau=\sum_{i, j, k=1}^{n}\left(b_{i j} \frac{2 u_{x_{k}} u_{x_{k} x_{j}}}{|\nabla u|^{2}}\right)_{x_{i}}=\sum_{i, j, k=1}^{n} \frac{2 u_{x_{k}}}{|\nabla u|^{2}}\left(b_{i j} u_{x_{k} x_{j}}\right)_{x_{i}}+\sum_{i, j, k=1}^{n} 2 b_{i j} u_{x_{k} x_{j}}\left(\frac{u_{x_{k}}}{|\nabla u|^{2}}\right)_{x_{i}} .
$$

The first term on the right is zero since $L u_{x_{k}}=0$. We differentiate the second term to get

$$
L \tau=\sum_{i, j, k=1}^{n}\left[2|\nabla u|^{-2} b_{i j} u_{x_{k} x_{j}} u_{x_{k} x_{i}}-\sum_{i, j, k, l=1}^{n} 4|\nabla u|^{-4} u_{x_{k}} u_{x_{k} x_{j}} b_{i j} u_{x_{l}} u_{x_{l} x_{i}}\right] .
$$

Evaluating at $a$, and using that $u_{x_{j}}(a)=0$ for $j \neq 1$, we see that

$$
b_{11}=(p-1)|\nabla u|^{p-2}, \quad b_{i i}=|\nabla u|^{p-2} \quad \text { for } i \neq 1, \quad b_{i j}=0 \quad \text { for } i \neq j .
$$

Using these equalities in (5.5) we obtain, at $a \in \Omega$,

$$
L \tau=2|\nabla u|^{p-4}\left((p-1) \sum_{k=1}^{n} u_{x_{k} x_{1}}^{2}+\sum_{i=2, k=1}^{n} u_{x_{k} x_{i}}^{2}-2(p-1) u_{x_{1} x_{1}}^{2}-\sum_{i=2}^{n} 2 u_{x_{1} x_{i}}^{2}\right) .
$$

Collecting the $x_{1} x_{1}$ and $x_{1} x_{i}(i \neq 1)$ derivatives yields

$$
L \tau=2|\nabla u|^{p-4}\left(-(p-1) u_{x_{1} x_{1}}^{2}+(p-2) \sum_{k=2}^{n} u_{x_{k} x_{1}}^{2}+\sum_{k, i=2}^{n} u_{x_{k} x_{i}}^{2}\right) .
$$

The last sum contains the pure second derivatives of $u$ in the $e_{k}$ direction when $k \neq 1$. These derivatives may be estimated using the $p$-Laplace equation for $u$ at the point $a$, i.e., at $a$ we have

$$
(p-1) u_{x_{1} x_{1}}+\sum_{k=2}^{n} u_{x_{k} x_{k}}=0 .
$$

Solving for $u_{x_{1} x_{1}}$, taking squares and using Hölder's inequality we see that

$$
\sum_{k=2}^{n} u_{x_{k} x_{k}}^{2} \geq \frac{(p-1)^{2}}{n-1} u_{x_{1} x_{1}}^{2} .
$$


Substituting this expression into (5.6) gives

$$
L \tau \geq 2|\nabla u|^{p-4}\left(\left(\frac{(p-1)^{2}}{n-1}-(p-1)\right) u_{x_{1} x_{1}}^{2}+(p-2) \sum_{k=2}^{n} u_{x_{k} x_{1}}^{2}+\sum_{k, i=2, k \neq i}^{n} u_{x_{k} x_{i}}^{2}\right) .
$$

Thus, $L \tau \geq 0$ when $\frac{(p-1)^{2}}{n-1}-(p-1)=\frac{(p-1)(p-n)}{n-1} \geq 0$. In particular, $L \tau \geq 0$ if $p \geq n$.

We note, for later use, that the above argument shows, for $p>n$, that

$$
L \tau \geq c^{-1}|\nabla u|^{p-4} \sum_{i, j=1}^{n} u_{x_{i} x_{j}}^{2}
$$

for some $c=c(p, n)$, where $c \rightarrow \infty$ as $p \rightarrow n$.

Proof of Theorem 1. To prove Theorem 1, let $\mu$ be the positive Borel measure associated with $u$. Using Lemma 3.6, Harnack's inequality, and the NTA property of $\Omega$, we see that there exist $c \geq 1$ and $\theta>0$, both depending only on $p, n$, such that

$$
c^{-1}\left(\frac{s}{r}\right)^{1 / \theta} \leq \frac{\mu(B(y, s))}{\mu(B(w, r))} \leq c\left(\frac{s}{r}\right)^{\theta}
$$

whenever $w \in \partial \Omega, y \in \partial \Omega \cap B(w, r)$, and $0<s<r$. Let $\hat{K}$ be the set of all $x \in \partial \Omega$ with

$$
\limsup _{r \rightarrow 0} \frac{\mu(B(x, r))}{r^{n-1}}>0 .
$$

We claim that

$$
\hat{K} \text { has } \sigma \text {-finite } H^{n-1} \text {-measure. }
$$

Indeed, given $m$ a positive integer, let $\hat{K}_{m}$ be the subset of $\hat{K} \cap B(0, m)$ for which the lim sup in (5.9) is $>1 / m$. Using a well known covering lemma, we can choose a covering $\left\{B\left(y_{i}, 5 r_{i}\right)\right\}$ of $\hat{K}_{m} \cap B(w, r)$ with $y_{i} \in \hat{K}, r_{i} \leq \epsilon<1 / 5,\left\{B\left(y_{i}, r_{i}\right)\right\}$ pairwise disjoint and

$$
\mu\left(B\left(y_{i}, 5 r_{i}\right)\right)>5 r_{i}^{n-1} / m
$$

Thus

$$
\begin{aligned}
\sum_{i} r_{i}^{n-1} & <m \sum_{i} \mu\left(B\left(x_{i}, 5 r_{i}\right)\right) \leq c m \sum_{i} \mu\left(B\left(x_{i}, r_{i}\right)\right) \\
& \leq c m \mu(\partial \Omega \cap B(0,2 m))<\infty
\end{aligned}
$$

as we see from (5.11), (5.8). Letting $\epsilon \rightarrow 0$ and using the definition of $H^{n-1}$-measure we conclude from (5.12) that $H^{n-1}\left(\hat{K}_{m}\right)<\infty$. Hence (5.10) is true.

To complete the proof of Theorem 1 we intend to show that

$$
\mu(\partial \Omega \backslash \hat{K})=0
$$


and the proof is by contradiction. Indeed, assume that (5.13) does not hold. Then, if $w \in \partial \Omega \backslash \hat{K}$, it follows from (5.4) and Theorem 3.7 that

$$
|\nabla u(x)| \approx r^{-1} u\left(a_{r}(w)\right) \approx\left(r^{1-n} \mu[B(w, r)]\right)^{1 /(p-1)} \rightarrow 0 \quad \text { as } r \rightarrow 0
$$

for $x \in B\left[a_{r}(w), d\left(a_{r}(w), \partial \Omega\right) / 2\right]$. Clearly (5.14) implies that $\tau(x) \rightarrow-\infty$ as $x \rightarrow \partial \Omega$ in the sense stated. We will use this fact to construct $\hat{\Omega} \subset \Omega$ and a compact set $F \subset$ $\partial \hat{\Omega} \cap(\partial \Omega \backslash \hat{K})$ such that $\tau$ is bounded from above in $\hat{\Omega}$ and $\tau(x) \rightarrow-\infty$ whenever $x \rightarrow y \in F$. Furthermore, by construction we will have $\mu(F)>0$. Indeed, to start the construction of $\hat{\Omega}$ and $F$ we see that if (5.13) does not hold, then by a measure-theoretic argument there exist $w \in \partial \Omega, \rho>0$ small, and $F$ compact such that

$$
F \subset(\partial \Omega \backslash \hat{K}) \cap B(w, \rho) \text { and } \mu(F)>0 .
$$

We construct $\hat{\Omega}$ based on $F$. Let $x \in F$ and let $k$ be an integer with $2^{k} \leq 100 \rho$. Let $P=P\left(x, 2^{k+10 n}\right)$ be the hyperplane corresponding to $x, 2^{k+10 n}$, in Definition 2.1 . Let $\pi(z)$ be the orthogonal projection of $z$ onto $P$ and let $\eta=\eta\left(x, 2^{k}\right)$ be the unit normal to $P$ pointing into $\Omega$. Let

$$
C(x, k)=\left\{z:|\pi(z)|<1000 \cdot 2^{k} \text { and } 2^{k-4}<\langle z, \eta\rangle<2^{k+4}\right\}
$$

and put

$$
\hat{\Omega}=\bigcup_{2^{k} \leq 100 \rho, x \in F} C(x, k) .
$$

Using $\delta$-Reifenberg flatness of $\Omega$, it is easily shown, for $\delta$ small enough, that $\hat{\Omega} \subset \Omega$ is an NTA-domain. By construction,

$$
\tau(x) \rightarrow-\infty \quad \text { as } x \in \hat{\Omega} \rightarrow z \in F \subset \partial \hat{\Omega} .
$$

Let $\theta(z)=\lim \sup _{x \in \hat{\Omega} \rightarrow z} \tau(x)$ whenever $z \in \partial \hat{\Omega}$. From (5.17) and a compactness argument we see that $\tau$ is bounded from above in $\hat{\Omega}$. Thus $\theta$ is upper semicontinuous on $\partial \hat{\Omega}$ and there exists a decreasing sequence $\left(\theta_{n}\right)$ of continuous functions with $\lim _{n \rightarrow \infty} \theta_{n}=\theta$ pointwise on $\partial \hat{\Omega}$. Let $\Theta_{n}$ be the solution to the Dirichlet problem for $L, \hat{\Omega}$, with boundary function $\theta_{n}$. Since $\tau$ is a subsolution to $L$ (see Lemma 5.1) and $\theta \leq \theta_{n}$, it follows from the weak maximum principle for $L$ that $\tau \leq \Theta_{n}$. From this inequality, (4.9), and the monotone convergence theorem we conclude that

$$
\tau\left(a_{\rho}(w)\right) \leq \lim _{n \rightarrow \infty} \Theta_{n}\left(a_{\rho}(w)\right)=\lim _{n \rightarrow \infty} \int \Theta_{n} d \hat{\omega}\left(\cdot, a_{\rho}(w)\right)=\int \theta d \hat{\omega}\left(\cdot, a_{\rho}(w)\right) .
$$

Here $a_{\rho}(w)$ is defined relative to $\hat{\Omega}$. Since $\theta=-\infty$ on $F$ we conclude from (5.18) that

$$
\hat{\omega}\left(F, a_{\rho}(w)\right)=0
$$

where $\hat{\omega}\left(\cdot, a_{\rho}(w)\right)$ is the elliptic measure, at $a_{\rho}(w)$, associated to $L$ and $\hat{\Omega}$. Furthermore, let $g$ be the Green function for $\Omega$ relative to $L$, and $\omega$ the corresponding elliptic measure. Then, using (5.19) and Lemma 4.5 it follows that

$$
\omega\left(F, a_{\rho}(w)\right)=0 .
$$


Finally from Lemmas 4.3, 4.4, 4.6, 3.6, and Theorem 3.7, we deduce that

$$
\mu(B(z, s)) \approx \omega\left(B(z, s), a_{\rho}(w)\right) .
$$

Here $\approx$ means that the constants can be chosen independent of $s, z$, provided $0<s \leq \rho / c$ and $z \in \partial \Omega \cap B(w, \rho)$. Using (5.21) and a covering argument we first find that $\mu$ restricted to $\partial \Omega \cap B(w, \rho)$ is absolutely continuous with respect to $\omega\left(\cdot, a_{\rho}(w)\right)$ and thereupon from (5.20) that $\mu(F)=0$. In particular, we have reached a contradiction to (5.15). Thus (5.13) is valid and the proof of Theorem 1 is complete.

\section{Estimates of Wolff type integrals - the case of an unbounded Lipschitz domain}

\subsection{Existence and uniqueness of $\hat{u}$}

Let $C=\left\{x:\left|x_{i}\right| \leq 1 / 2,1 \leq i \leq n\right\}$. Let $\hat{\Omega}$ be an NTA-domain with constants $M, r_{0}=\infty$ such that $\partial \hat{\Omega} \cap\left(\mathbb{R}^{n} \backslash C\right)=\left\{x: x_{n}=0\right\} \cap\left(\mathbb{R}^{n} \backslash C\right)$. We prove

Lemma 6.1. For given $p \in(1, \infty)$, there exists a unique positive $p$-harmonic function $\hat{u}$ in $\hat{\Omega}$, continuous in $\mathbb{R}^{n}$, such that

$$
\hat{u} \equiv 0 \quad \text { on } \mathbb{R}^{n} \backslash \hat{\Omega} \quad \text { and } \quad\left|x_{n}-\hat{u}(x)\right| \rightarrow 0 \quad \text { uniformly as }|x| \rightarrow \infty, x \in \hat{\Omega} .
$$

Proof. We first establish the existence part of Lemma 6.1. Indeed, suppose $\rho \gg n$ and let $u_{\rho}(\cdot)$ be the $p$-harmonic function in $B(0, \rho) \cap \hat{\Omega}$ with boundary values (in the Sobolev sense) $u_{\rho}=x_{n}$ on $\partial B(0, \rho) \cap \hat{\Omega}$ and $u_{\rho}=0$ on $\bar{B}(0, \rho) \cap \partial \hat{\Omega}$. Existence of $u_{\rho}(\cdot)$ follows from the usual calculus of variations minimizing argument for a functional involving the gradient raised to the $p$ th power. From the maximum principle for $p$-harmonic functions on $\hat{\Omega} \cap B(0, \rho)$ we have

$$
\left|x_{n}-u_{\rho}(x)\right| \leq 1, \quad x \in \hat{\Omega} \cap B(0, \rho) .
$$

From Lemma 3.3 we see that $u_{\rho}(\cdot)$ is Hölder continuous in $B(0, \rho)$ with exponent independent of $\rho$. Using Ascoli's theorem and Lemma 3.5 it follows that a subsequence, $\left\{u_{\rho_{m}}(\cdot)\right\}$, converges uniformly on compact subsets of $\mathbb{R}^{n}$ (extend $u_{\rho}(\cdot)$ to all of $B(0, \rho)$ by setting it equal to zero in $\left.B(0, \rho) \cap\left(\mathbb{R}^{n} \backslash \hat{\Omega}\right)\right)$ to a Hölder continuous function $\hat{u} \geq 0$ on $\mathbb{R}^{n}$ which is $p$-harmonic in $\hat{\Omega}$. Also (6.1) holds with $u_{\rho}(\cdot)$ replaced by $\hat{u}$, for $x \in \hat{\Omega}$. To proceed we apply a barrier argument, Lemmas $3.5,3.4$ to $\hat{u}$, to get

$$
|\nabla \hat{u}(x)| \leq c^{\prime} \hat{u}\left(|x| e_{n}\right) /|x| \leq c \quad \text { for } x \in \hat{\Omega},|x| \geq 2 n,
$$

where $e_{n}=(0, \ldots, 0,1)$. Next if $\xi=\left(\xi_{1}, \ldots, \xi_{n}\right), w=\left(w_{1}, \ldots, w_{n}\right) \in \mathbb{R}^{n} \backslash\{0\}$, and $1 \leq i \leq n$, we note that

$$
\begin{aligned}
|\xi|^{p-2} \xi_{i}-|w|^{p-2} w_{i} & =\int_{0}^{1} \frac{d}{d \lambda}\left\{|\lambda \xi+(1-\lambda) w|^{p-2}\left[\lambda \xi_{i}+(1-\lambda) w_{i}\right]\right\} d \lambda \\
& =\sum_{j=1}^{n}(\xi-w)_{j}\left(\int_{0}^{1} a_{i j}[\lambda \xi+(1-\lambda) w] d \lambda\right),
\end{aligned}
$$


where, for $1 \leq i, j \leq n$,

$$
a_{i j}(\eta)=|\eta|^{p-4}\left[(p-2) \eta_{i} \eta_{j}+\delta_{i j}|\eta|^{2}\right] \quad \text { for } \eta \in \mathbb{R}^{n} \backslash\{0\} .
$$

In this display $\delta_{i j}$, once again, denotes the Kronecker delta. Using (6.3) and the fact that $\hat{u}, x_{n}$ are $p$-harmonic we deduce that if $U(x)=x_{n}-\hat{u}(x)$, and

$$
A_{i j}(x)=\int_{0}^{1} a_{i j}\left[\lambda e_{n}+(1-\lambda) \nabla \hat{u}(x)\right] d \lambda
$$

whenever $x \in \hat{\Omega}$ and $1 \leq i, j \leq n$, then

$$
\tilde{L} U(x)=\sum_{i, j=1}^{n} \frac{\partial}{\partial x_{i}}\left[A_{i j}(x) U_{x_{j}}\right]=0 \quad \text { on } \hat{\Omega} .
$$

Moreover, for $x \in \hat{\Omega}$, it is easily seen that

$$
\begin{aligned}
& c^{-1}|\xi|^{2}(1+|\nabla \hat{u}(x)|)^{p-2} \leq \sum_{i, j=1}^{n} A_{i j}(x) \xi_{i} \xi_{j} \quad \text { whenever } \xi \in \mathbb{R}^{n} \backslash\{0\}, \\
& \sum_{i, j=1}^{n}\left|A_{i j}(x)\right| \leq c(1+|\nabla \hat{u}(x)|)^{p-2},
\end{aligned}
$$

where $c$ depends only on $p, n$, and can be chosen independent of $p$ when $p \in[3 / 2,5 / 2]$. From (6.5), (6.6) we see that $U$ is a solution in $\hat{\Omega}$ to $\tilde{L}$, a degenerate elliptic PDE whose ellipticity is given by (6.6). Moreover from (6.2), $\tilde{L}$ is uniformly elliptic with bounded coefficients in $\hat{\Omega} \backslash \bar{B}(0,2 n)$. Using (4.8) with $\lambda=1$, (6.1), (6.6), Lemma 4.1(ii), and the maximum principle for solutions to $\tilde{L}$, we see that if $\tilde{\omega}_{\rho}$ is elliptic measure for $\tilde{L}$ and $\tilde{\Omega}_{\rho}:=(\hat{\Omega} \cap B(0,2 \rho)) \backslash \bar{B}(0,2 n)$, defined as in (4.9), then

$$
\begin{array}{r}
\left|x_{n}-\hat{u}(x)\right| \leq \tilde{\omega}_{\rho}\left(\partial \tilde{\Omega}_{\rho} \cap \partial B(0,2 \rho), x\right)+\tilde{\omega}_{\rho}\left(\partial \tilde{\Omega}_{\rho} \cap \partial B(0,2 n), x\right) \\
\text { for } 2 n<|x|<2 \rho,
\end{array}
$$

as follows by comparing boundary values and using (6.1) for $\hat{u}$. Now using Lemma 4.2(iii) we observe that

$$
\tilde{\omega}_{\rho}\left(\partial \tilde{\Omega}_{\rho} \cap \partial B(0,2 \rho), x\right)=1-\tilde{\omega}_{\rho}\left(\partial \tilde{\Omega}_{\rho} \backslash \partial B(0,2 \rho), x\right) \leq c(|x| / \rho)^{\alpha}
$$

whenever $8 n<|x|<\rho / 4, x \in \tilde{\Omega}$. Moreover, let $\tilde{g}_{\rho}(\cdot, \cdot)$ be the Green function for $\tilde{\Omega}, \tilde{L}$. Then using Lemmas 4.4, 4.1(ii) and 4.3(b), with $\lambda \equiv 1$, we see that

$$
\tilde{\omega}_{\rho}\left(\partial \tilde{\Omega}_{\rho} \cap \partial B(0,2 n), x\right) \leq c \tilde{g}_{\rho}\left(x, 3 n e_{n}\right) \leq c|x|^{2-n-\sigma} \quad \text { whenever } 8 n<|x|<\rho / 4 .
$$

Combining (6.7)-(6.9) we can conclude that

$$
\left|x_{n}-\hat{u}(x)\right| \leq c\left((|x| / \rho)^{\alpha}+|x|^{2-n-\sigma}\right) \quad \text { whenever } 8 n<|x|<\rho / 4 .
$$


The nonnegative constants $c, \alpha$, and $\sigma$ are independent of $\rho$ and $x$. Hence, if we let $\rho \rightarrow \infty$ through a sequence we then see that $U \rightarrow 0$ as $x \rightarrow \infty$ and existence of $\hat{u}$ in Lemma 6.1 has been established.

To prove uniqueness suppose $v$ is another such solution and let $\left\{u_{\rho_{m}}(\cdot)\right\}$ be the sequence used in the construction of $\hat{u}$ above. Then given $\epsilon>0$ we have, for $m$ sufficiently large,

$$
\left|u_{\rho_{m}}(\cdot)-v\right| \leq \epsilon \quad \text { in } \hat{\Omega} \cap B\left(0, \rho_{m}\right) .
$$

This follows from comparing the boundary values of $u_{\rho_{m}}$ and $v$, and by using the fact that $\left|x_{n}-v(x)\right| \rightarrow 0$ uniformly as $|x| \rightarrow \infty$. Letting $m \rightarrow \infty$ we conclude that $\hat{u}=v$. Hence $\hat{u}$ is unique and the proof of Lemma 6.1 is complete.

\subsection{Existence, differentiability, and sign of $I(\epsilon)$}

Now let $\hat{\theta} \in C_{0}^{\infty}\left(\left\{x^{\prime}:\left|x^{\prime}\right|<1 / 2\right\}\right)$ with $\|\hat{\theta}\|_{\infty}<1 / 2$ and set $\hat{\Omega}(\epsilon)=\left\{\left(x^{\prime}, x_{n}\right): x_{n}>\right.$ $\left.\epsilon \hat{\theta}\left(x^{\prime}\right), x^{\prime} \in \mathbb{R}^{n-1}\right\}$ for $\epsilon \in(0,1)$. For fixed $p \in(1, \infty)$, let $\hat{u}(\cdot, \epsilon)$, be the unique $p$ harmonic function function in $\hat{\Omega}(\epsilon)$ satisfying the conclusion Lemma 6.1. Put $U(x, \epsilon)=$ $x_{n}-\hat{u}(x, \epsilon)$ for $x \in \hat{\Omega}(\epsilon)$. We sometimes write $\hat{u}, U, \hat{\Omega}$, for $\hat{u}(\cdot, \epsilon), U(\cdot, \epsilon), \hat{\Omega}(\cdot, \epsilon)$.

Remark 6.2. For some $\eta \in(0,1)$ depending only on $p, n$ we have

$$
\hat{u}_{x_{n}}(x) \geq \eta|\nabla \hat{u}(x)| \geq \eta^{2} \hat{u}(x) / d(x, \partial \hat{\Omega}) \quad \text { for } x \in \hat{\Omega} .
$$

Indeed, the right-hand inequality in (6.11) is given in Theorem 3.7 when $\partial \hat{\Omega}$ is flat enough. However the above inequality holds with no flatness assumption in a Lipschitz graph domain provided $\eta$ is also allowed to depend on the Lipschitz norm of the graph function, and $\eta$ can be chosen independent of $p$ when $p \in[3 / 2,5 / 2]$ (see [LN2, Lemma 4.28]).

In the following we first establish the existence and differentiability of $I(\epsilon)$.

Existence of $I(\epsilon)$. From (6.11) and Lemma 3.5 we see that the coefficients $A_{i j}$ of $\tilde{L}$ in (6.5), defined with respect to $x_{n}$ and $\hat{u}=\hat{u}(\cdot, \epsilon)$, are infinitely differentiable in $\hat{\Omega}$, and that $U=U(\cdot, \epsilon)$ is a solution to this divergence form elliptic PDE (see (6.6)). Let $\tilde{g}(\cdot, \cdot), \tilde{\omega}(\cdot, \cdot)$, be the Green function and elliptic measure corresponding to $\tilde{L}$ in $\hat{\Omega}$. As in (6.10) we get

$$
|U(x)| \leq c^{\prime}|x|^{2-n-\sigma} \quad \text { in } \hat{\Omega} \backslash \bar{B}(0,8 n) .
$$

Extending $\hat{u}$ by Schwarz reflection, and using Schauder estimates, we see that

$$
\left|\nabla^{k} \hat{u}(x)\right| \leq c(k)|x|^{1-k} \quad \text { for }|x| \geq 8 n
$$

for $k=0,1, \ldots$, with $c(k)$ depending only on $p, n, k$. Furthermore, also extending $U$ by Schwarz reflection and using (6.12) we deduce that

$$
|\nabla U(x)| \leq c|x|^{-1} \max \{U(y):|x| / 2 \leq|y| \leq 3|x| / 2\} \leq \tilde{c}|x|^{1-n-\sigma}
$$


in $\mathbb{R}^{n} \backslash \bar{B}(0,8 n)$ where $\tilde{c} \geq 1$ depends only on $p, n$. Next let

$$
I=I(\epsilon)=\int_{\partial \hat{\Omega}(\epsilon)}|\nabla \hat{u}(\cdot, \epsilon)|^{p-1} \log |\nabla \hat{u}(\cdot, \epsilon)| d H^{n-1}
$$

and observe, using that $U(x, \epsilon)=x_{n}-\hat{u}(x, \epsilon),(6.13),(6.14)$ as well as first semester calculus, that

$$
\int_{\partial \hat{\Omega}(\epsilon) \cap\left[\mathbb{R}^{n} \backslash B(0,8 n)\right]}|\nabla \hat{u}(\cdot, \epsilon)|^{p-1}|\log | \nabla \hat{u}(\cdot, \epsilon)|| d H^{n-1} \leq c
$$

where $c$ depends only on $p, n$ and can be chosen independent of $p \in[3 / 2,5 / 2]$. Next we note from a result of Lieberman [Li] that $\nabla \hat{u}$ has a Hölder $\gamma$ extension to the closure of $\hat{\Omega} \cap B(0,8 n)$ for some $\gamma \in(0,1]$, depending on $p, n$, and the $C^{2}$ norm for $\hat{\theta}$. Using this result and (6.11) we conclude that $\hat{u}$ is a solution to a non-divergence form uniformly elliptic equation with Hölder continuous coefficients in the closure of $\hat{\Omega} \cap B(0,2 n)$. We now use classical Schauder theory (see [GT, Chapters 6, 9]) and a bootstrap type argument to find that $\hat{u}$ has a $C^{\infty}$ extension with $\nabla \hat{u} \neq 0$ to the closure of $\hat{\Omega} \cap B(0,8 n)$. In view of this fact and (6.16) we deduce the existence of $I(\epsilon)$.

Differentiability of $I(\epsilon)$. Let

$$
\hat{U}\left(x, \epsilon_{1}, \epsilon_{2}\right)=\frac{\hat{u}\left(x, \epsilon_{2}\right)-\hat{u}\left(x, \epsilon_{1}\right)}{\epsilon_{2}-\epsilon_{1}} .
$$

Note that $\hat{u}\left(x^{\prime}, \epsilon \hat{\theta}\left(x^{\prime}\right), \epsilon\right) \equiv 0$ for all $x^{\prime} \in \mathbb{R}^{n-1}$. Using this and Schauder estimates up to the boundary one can show that

$$
\max _{x \in \partial\left[\hat{\Omega}\left(\epsilon_{1}\right) \cap \hat{\Omega}\left(\epsilon_{2}\right)\right]}\left|\hat{U}\left(x, \epsilon_{1}, \epsilon_{2}\right)+\hat{\theta}\left(x^{\prime}\right) \hat{u}_{x_{n}}\left(x, \epsilon_{1}\right)\right| \rightarrow 0 \quad \text { as } \epsilon_{2} \rightarrow \epsilon_{1} .
$$

Thus $\hat{U}\left(\cdot, \epsilon_{1}, \epsilon_{2}\right)$ is uniformly bounded on $\partial\left[\hat{\Omega}\left(\epsilon_{2}\right) \cap \hat{\Omega}\left(\epsilon_{1}\right)\right]$ and $\equiv 0$ on $\partial \hat{\Omega}(\cdot) \cap\left(\mathbb{R}^{n} \backslash\right.$ $B(0, n))$. From this fact and our earlier estimates we conclude that $\hat{U}\left(\cdot, \epsilon_{1}, \epsilon_{2}\right)$ is a bounded solution to an elliptic PDE (constructed similarly to the one in (6.4), (6.5)) with ellipticity constants independent of $\epsilon_{1}, \epsilon_{2} \in\left[-\epsilon_{0}, \epsilon_{0}\right]$, and with $C^{\infty}$ coefficients. Also from (6.10) for $\hat{u}\left(\cdot, \epsilon_{i}\right), i=1,2$, we find that $\hat{U}\left(x, \epsilon_{1}, \epsilon_{2}\right) \rightarrow 0$ uniformly as $|x| \rightarrow \infty$. Using these facts and arguing as in (6.10) we conclude, for some $\tilde{\sigma} \in(0,1)$, and $C \geq 1$, independent of $\epsilon_{1}, \epsilon_{2}$, that

$$
\left|\hat{U}\left(x, \epsilon_{1}, \epsilon_{2}\right)\right| \leq C|x|^{2-n-\tilde{\sigma}} \quad \text { for } x \in \hat{\Omega}\left(\epsilon_{1}\right) \backslash B(0, n) .
$$

Letting $\epsilon_{2} \rightarrow \epsilon_{1}$ it again follows from Schauder type estimates (see for example (4.11)(4.16) in [LN2]) that the derivative of $\hat{u}(\cdot, \epsilon)$ with respect to $\epsilon, \hat{u}_{\epsilon}(\cdot, \epsilon)$, exists at $\epsilon_{1}$ and that

$$
\hat{u}_{\epsilon}\left(x, \epsilon_{1}\right)=-\hat{\theta}\left(x^{\prime}\right) \hat{u}_{x_{n}}\left(x, \epsilon_{1}\right) \quad \text { whenever } x \in \partial \hat{\Omega}\left(\epsilon_{1}\right) .
$$

Furthermore, $\hat{u}_{\epsilon}$ is, at $\epsilon=\epsilon_{1}$, a $C^{\infty}$ solution in $\hat{\Omega}\left(\epsilon_{1}\right)$ to

$$
L \zeta(x)=\sum_{i, j=1}^{n} \frac{\partial}{\partial x_{i}}\left[b_{i j}(x, \epsilon) \zeta_{x_{j}}(x)\right]=0,
$$


where

$$
b_{i j}(x, \epsilon)=|\nabla \hat{u}(x, \epsilon)|^{p-4}\left[(p-2) \hat{u}_{x_{i}}(x, \epsilon) \hat{u}_{x_{j}}(x, \epsilon)+\delta_{i j}|\nabla \hat{u}(x, \epsilon)|^{2}\right]
$$

for $1 \leq i, j \leq n$. Using a similar argument for derivatives of $\hat{u}(\cdot, \epsilon)$ we deduce that $\left(\nabla^{k} \hat{u}\right)_{\epsilon}=\nabla^{k}\left(\hat{u}_{\epsilon}\right)$, where $\nabla^{k}$ for $k \geq 2$ denotes an arbitrary $k$ th partial derivative in $x$. Also from (6.17)-(6.20), Schwarz reflection and Schauder estimates we get

$$
\left|\nabla^{k} \hat{u}_{\epsilon}\right| \leq C(k)|x|^{2-n-\tilde{\sigma}-k}
$$

for $|x|>8 n$. Continuing, using difference quotients and Schauder type arguments, we find, for $\epsilon \in\left[-\epsilon_{0}, \epsilon_{0}\right]$, that $\hat{u}_{\epsilon^{2}}$ (the second order derivative of $\hat{u}(\cdot, \epsilon)$ with respect to $\epsilon$ ) is $C^{\infty}$ in the closure of $\hat{\Omega}(\epsilon)$ and that

$$
L \hat{u}_{\epsilon^{2}}(x)=-\sum_{i, j=1}^{n} \frac{\partial}{\partial x_{i}}\left[\left(b_{i j}\right)_{\epsilon}\left(\hat{u}_{\epsilon}\right)_{x_{j}}\right](x, \epsilon)
$$

with

$$
\hat{u}_{\epsilon^{2}}=-2 \hat{\theta}\left(x^{\prime}\right)\left(\hat{u}_{\epsilon}\right)_{x_{n}}-\hat{\theta}\left(x^{\prime}\right)^{2} \hat{u}_{x_{n}^{2}} \quad \text { at } x=\left(x^{\prime}, \epsilon \hat{\theta}\left(x^{\prime}\right)\right) \in \partial \hat{\Omega}(\epsilon) .
$$

Moreover

$$
D^{l} \hat{u} \text { is continuous on the closure of } \bigcup_{\epsilon \in\left[-\epsilon_{0}, \epsilon_{0}\right]} \hat{\Omega}(\epsilon) \times\{\epsilon\}
$$

where $D^{l}$ denotes any combination of partial derivatives in $x, \epsilon$ with at most two partial derivatives in $\epsilon$. Finally (6.21) holds with $\hat{u}_{\epsilon}$ replaced by $\hat{u}_{\epsilon^{2}}$. Furthermore, proceeding by induction we deduce that (6.24) holds for any combination of partial derivatives in $x, \epsilon$. Also (6.21) holds with $\hat{u}_{\epsilon}$ replaced by $\hat{u}_{\epsilon}$, the derivative of order $m$ of $\hat{u}(\cdot, \epsilon)$ with respect to $\epsilon$, and $C(k)$ replaced by $C(k, m)$ whenever $m$ is a positive integer. Also, analogues of (6.22), (6.23) are valid, as can be obtained from differentiating these equations with respect to $\epsilon$. Thus $I(\epsilon)$ as defined in (6.15) is in $C^{\infty}\left[-\epsilon_{0}, \epsilon_{0}\right]$ and derivatives can be found by differentiating under the integral sign.

The sign of $I(\epsilon)$. Concerning the sign of $I(\epsilon)$ we prove

Lemma 6.3. For I as in (6.15) and p fixed, $1<p<\infty$, we have

$$
I(0)=0, \quad I^{\prime}(0)=0, \quad \text { and } \quad I^{\prime \prime}(0)=\frac{p-2}{p-1} \int_{\mathbb{R}^{n-1}}\left|\nabla^{\prime} \hat{\theta}\right|^{2}\left(x^{\prime}\right) d x^{\prime} .
$$

In Lemma $6.3, \nabla^{\prime}$ denotes the gradient on $\mathbb{R}^{n-1}$ with respect to $x^{\prime}$ and $d x^{\prime}$ is the $n-1$ Lebesgue measure. Note that once Lemma 6.3 is proved it follows from Taylor's theorem with remainder that, for fixed $p \neq 2$, there exists $\epsilon_{1}$, depending on $p, n$, and the $C^{3}\left(\mathbb{R}^{n-1}\right)$ norm of $\hat{\theta}$, such that $I(\epsilon)>0$ for $p>2$ and $I(\epsilon)<0$ for $p<2$ in $\left[-\epsilon_{1}, \epsilon_{1}\right]$. For $p=2$ the sign of $I$ depends on $\hat{\theta}$, as discussed in Section 2 . 
Proof of Lemma 6.3. To begin, we observe that

$$
I(\epsilon)=\int_{\mathbb{R}^{n-1}}\left|\nabla \hat{u}\left(x^{\prime}, \epsilon \hat{\theta}\left(x^{\prime}\right), \epsilon\right)\right|^{p-1} \log \left|\nabla \hat{u}\left(x^{\prime}, \epsilon \hat{\theta}\left(x^{\prime}\right), \epsilon\right)\right| \sqrt{\left.1+\epsilon^{2} \mid \nabla^{\prime} \hat{\theta}\left(x^{\prime}\right)\right)\left.\right|^{2}} d x^{\prime} .
$$

Also

$$
\frac{\left(-\epsilon \nabla^{\prime} \hat{\theta}\left(x^{\prime}\right), 1\right)}{\left(1+\epsilon^{2}\left|\nabla^{\prime} \hat{\theta}\right|^{2}\right)^{1 / 2}}
$$

is the inner normal to $\partial \Omega(\epsilon)$ at $\left(x^{\prime}, \epsilon \hat{\theta}\left(x^{\prime}\right)\right)$, and $\nabla \hat{u}\left(x^{\prime}, \epsilon \hat{\theta}\left(x^{\prime}\right), \epsilon\right)$ is parallel to this inner normal. Thus at $\left(x^{\prime}, \epsilon \hat{\theta}\left(x^{\prime}\right)\right)$,

$$
|\nabla \hat{u}|=\frac{-\epsilon\left\langle\nabla^{\prime} \hat{\theta}, \nabla^{\prime} \hat{u}\right\rangle+\hat{u}_{x_{n}}}{\sqrt{1+\epsilon^{2}\left|\nabla^{\prime} \hat{\theta}\right|^{2}}},
$$

where $\langle\cdot, \cdot\rangle$ denotes the usual inner product on $\mathbb{R}^{n-1}$. Substituting the last expression into the integrand in (6.25) we see that this integrand equals

$$
\begin{aligned}
{\left[-\epsilon\left\langle\nabla^{\prime} \hat{\theta}, \nabla^{\prime} \hat{u}\right\rangle+\right.} & \left.\hat{u}_{x_{n}}\right]^{p-1}\left(1+\epsilon^{2}\left|\nabla^{\prime} \hat{\theta}\right|^{2}\right)^{(2-p) / 2} \\
& \times\left\{\log \left(-\epsilon\left\langle\nabla^{\prime} \hat{\theta}, \nabla^{\prime} \hat{u}\right\rangle+\hat{u}_{x_{n}}\right)-\frac{1}{2} \log \left(1+\epsilon^{2}\left|\nabla^{\prime} \hat{\theta}\left(x^{\prime}\right)\right|^{2}\right)\right\} .
\end{aligned}
$$

Now from $\hat{u}(x, \epsilon)=0$ when $x=\left(x^{\prime}, \epsilon \hat{\theta}\left(x^{\prime}\right)\right) \in \partial \hat{\Omega}(\epsilon)$ and the chain rule we have

$$
\nabla^{\prime} \hat{u}=-\epsilon \hat{u}_{x_{n}} \nabla^{\prime} \hat{\theta} \quad \text { at } x=\left(x^{\prime}, \epsilon \hat{\theta}\left(x^{\prime}\right)\right) \in \partial \hat{\Omega}(\epsilon) .
$$

Using (6.26), (6.27), the integrand in (6.25) becomes, at $x=\left(x^{\prime}, \epsilon \hat{\theta}\left(x^{\prime}\right)\right)$,

$$
\left.F\left(x^{\prime}, \epsilon\right)=\left(1+\epsilon^{2} \mid \nabla^{\prime} \hat{\theta}^{2}\right)^{p / 2} \hat{u}_{x_{n}}^{p-1}\left\{\log \left(\hat{u}_{x_{n}}\right)+\left.\frac{1}{2} \log \left(1+\epsilon^{2} \mid \nabla^{\prime} \hat{\theta}\right)\right|^{2}\right)\right\} .
$$

We expand this expression in powers of $\epsilon$, since our goal is to find $I^{\prime \prime}(0)$. For this purpose we note from (6.21), (6.24), Taylor's theorem, and the fact that $\hat{u}(x, 0)=x_{n}$ that

$$
\hat{u}_{x_{n}}\left(x^{\prime}, \epsilon \hat{\theta}\left(x^{\prime}\right), \epsilon\right)=1+a_{1}\left(x^{\prime}\right) \epsilon+a_{2}\left(x^{\prime}\right) \epsilon^{2}+O\left(\epsilon^{3}\right),
$$

where $O\left(\epsilon^{3}\right)$ denotes a measurable function on $\mathbb{R}^{n-1}$ whose $L^{1}$ and $L^{\infty}$ norms are bounded by $A \epsilon^{3}$. Here $A$ is a constant independent of $\epsilon$ when $\epsilon \in\left[-\epsilon_{0}, \epsilon_{0}\right]$. Using this equality in (6.28) and once again (6.21), (6.24), we obtain, after expanding in powers of $\epsilon$,

$$
F\left(x^{\prime}, \epsilon\right)=a_{1}\left(x^{\prime}\right) \epsilon+\left(a_{2}\left(x^{\prime}\right)+(p-3 / 2) a_{1}\left(x^{\prime}\right)^{2}+\left|\nabla^{\prime} \theta\left(x^{\prime}\right)\right|^{2} / 2\right) \epsilon^{2}+O\left(\epsilon^{3}\right) .
$$

Using (6.29) in the integral defining $I(\epsilon)$, and letting $\epsilon \rightarrow 0$, we deduce that

$$
I^{\prime}(0)=\int_{\mathbb{R}^{n-1}} a_{1}\left(x^{\prime}\right) d x^{\prime},
$$

while

$$
I^{\prime \prime}(0)=\int_{\mathbb{R}^{n-1}}\left[2 a_{2}\left(x^{\prime}\right)+(2 p-3) a_{1}\left(x^{\prime}\right)^{2}+\left|\nabla^{\prime} \hat{\theta}\left(x^{\prime}\right)\right|^{2}\right] d x^{\prime}
$$


Note that $a_{1}, 2 a_{2}$ are the first and second total derivatives in $\epsilon$ of $\hat{u}_{x_{n}}\left(x^{\prime}, \epsilon \hat{\theta}\left(x^{\prime}\right), \epsilon\right)$ evaluated at $\epsilon=0$. Using this fact and smoothness of the derivatives of $\hat{u}$ in $(x, \epsilon)$ we see that $a_{1}\left(x^{\prime}\right)=\hat{u}_{\epsilon x_{n}}\left(x^{\prime}, 0\right)$. Now from (6.18), $\hat{u}(x, 0)=x_{n},(6.22)$, and (6.20), we deduce that

$$
\begin{aligned}
& \hat{u}_{\epsilon}\left(x^{\prime}, 0,0\right)=-\hat{\theta}\left(x^{\prime}\right), \quad x^{\prime} \in \mathbb{R}^{n-1}, \\
& (p-1) \hat{u}_{\epsilon x_{n}^{2}}\left(x^{\prime}, x_{n}, 0\right)+\sum_{i, j=1}^{n-1} \hat{u}_{\epsilon x_{i}^{2}}\left(x^{\prime}, x_{n}, 0\right)=0 \quad \text { for }\left(x^{\prime}, x_{n}\right) \in H,
\end{aligned}
$$

where $H:=\mathbb{R}^{n-1} \times(0, \infty)$. It follows from (6.32) that $h(x)=\hat{u}_{\epsilon}\left(x^{\prime}, \sqrt{p-1} x_{n}, 0\right)$ is harmonic on $H$ with boundary values $-\hat{\theta}$. Using the divergence theorem we can use (6.30) to conclude that

$$
I^{\prime}(0)=\int_{\mathbb{R}^{n-1}} a_{1}\left(x^{\prime}\right) d x^{\prime}=\int_{\mathbb{R}^{n-1}} \hat{u}_{\epsilon x_{n}} d x^{\prime}=(p-1)^{-1 / 2} \int_{\mathbb{R}^{n-1}} h_{x_{n}} d x^{\prime}=0 .
$$

Next we compute

$$
a_{2}\left(x^{\prime}\right)=2 \hat{\theta}\left(x^{\prime}\right) \hat{u}_{\epsilon x_{n}^{2}}\left(x^{\prime}, 0\right)+\hat{u}_{\epsilon^{2} x_{n}}\left(x^{\prime}, 0\right), \quad x^{\prime} \in \mathbb{R}^{n-1} .
$$

Observe that, from (6.32) and (6.24),

$$
\int_{\mathbb{R}^{n-1}} 2 \hat{\theta} \hat{u}_{\epsilon x_{n}^{2}} d x^{\prime}=\frac{2}{p-1} \sum_{i=1}^{n-1} \int_{\mathbb{R}^{n-1}} \hat{\theta} \hat{\theta}_{x_{i}^{2}} d x^{\prime}=-\frac{2}{p-1} \int_{\mathbb{R}^{n-1}}\left|\nabla^{\prime} \hat{\theta}\right|^{2} d x^{\prime} .
$$

Using (6.35), (6.34) in (6.31) we find that

$$
\begin{aligned}
I^{\prime \prime}(0) & =\int_{\mathbb{R}^{n-1}}\left[2 a_{2}\left(x^{\prime}\right)+(2 p-3) a_{1}\left(x^{\prime}\right)^{2}+\left|\nabla^{\prime} \hat{\theta}\left(x^{\prime}\right)\right|^{2}\right] d x^{\prime} \\
& =\int_{\mathbb{R}^{n-1}}\left[(2 p-3) \hat{u}_{\epsilon x_{n}}^{2}+\frac{p-3}{p-1}\left|\nabla^{\prime} \hat{\theta}\right|^{2}+\hat{u}_{\epsilon^{2} x_{n}}\right] d x^{\prime} .
\end{aligned}
$$

Observe that $\hat{u}_{\epsilon^{2}}$, evaluated at $\epsilon=0$, is a solution to the PDE in (6.22) on $H$. We integrate both sides of this equation over $H$. Next we integrate by parts to obtain an integral over $\partial H$ (permissible from the decay estimates in (6.21)). Finally using (6.20), (6.32) to evaluate coefficients at $\epsilon=0$, we get

$$
\int_{\mathbb{R}^{n-1}}\left[(p-1) \hat{u}_{\epsilon^{2} x_{n}}+(p-2)\left|\nabla^{\prime} \hat{\theta}\right|^{2}+(p-2)(p-1) \hat{u}_{\epsilon x_{n}}^{2}\right] d x^{\prime}=0 .
$$

Using (6.37) in (6.36) it follows that

$$
I^{\prime \prime}(0)=\int_{\mathbb{R}^{n-1}}\left[(p-1) \hat{u}_{\epsilon x_{n}}^{2}-(p-1)^{-1}\left|\nabla^{\prime} \hat{\theta}\right|^{2}\right] d x^{\prime} .
$$

Finally observe from harmonicity of $h$ defined above (6.33) and Rellich inequalities, or simply integration by parts, that

$$
\int_{\mathbb{R}^{n-1}}\left|\nabla^{\prime} \hat{\theta}\right|^{2} d x^{\prime}=(p-1) \int_{\mathbb{R}^{n-1}} \hat{u}_{\epsilon x_{n}}^{2} d x^{\prime}
$$


From (6.38), (6.39), we conclude

$$
I^{\prime \prime}(0)=\frac{p-2}{p-1} \int_{\mathbb{R}^{n-1}}\left|\nabla^{\prime} \theta\right|^{2} d x^{\prime} .
$$

This completes the proof of Lemma 6.3.

\subsection{Continuity and stability of $\operatorname{sgn} I$ under approximation}

Next in order to avoid confusion we change notation slightly. Given $\phi \in C_{0}^{\infty}\left(\left\{x^{\prime}\right.\right.$ : $\left.\left.\left|x^{\prime}\right|<1 / 2\right\}\right)$ with $C^{2}$ norm $\leq 1 / 100$, and $p \in(1, \infty)$, let $u^{\prime}=u^{\prime}(\cdot, p, \phi)$ be the unique positive $p$-harmonic function in $\Omega^{\prime}=\Omega^{\prime}(\phi)=\left\{\left(x^{\prime}, x_{n}\right): x_{n}>\phi\left(x^{\prime}\right)\right\}$ which is continuous in $\mathbb{R}^{n}$ with $u^{\prime} \equiv 0$ on $\mathbb{R}^{n} \backslash \Omega^{\prime}$ and $\lim _{|x| \rightarrow \infty}\left|x_{n}-u^{\prime}(x)\right|=0$ for $x \in \Omega^{\prime}$. Existence and uniqueness of $u^{\prime}$ follow from Lemma 6.1. Set

$$
I(\phi, p)=\int_{\partial \Omega}\left|\nabla u^{\prime}\right|^{p-1} \log \left|\nabla u^{\prime}\right| d H^{n-1} .
$$

Note that in the previous section we proved that $I(\phi, p)$ exists. In this subsection we prove the following propositions.

Proposition 6.4. With the above notation, we have $\lim _{p \rightarrow 2} I(\phi, p)=I(\phi, 2)$. Thus if $I(\phi, 2) \neq 0$, then there exists $\hat{\epsilon}=\hat{\epsilon}(\phi, n)>0$ such that $\operatorname{sgn} I(\phi, p)=\operatorname{sgn} I(\phi, 2)$ when $p \in[2-\hat{\epsilon}, 2+\hat{\epsilon}]$.

Proposition 6.5. There exists a sequence $\phi_{m}, m=1,2, \ldots$, of piecewise linear functions on $\mathbb{R}^{n-1}$ with $\left\|\phi_{m}\right\|_{\infty} \leq\|\phi\|_{\infty},\left\|\nabla \phi_{m}\right\|_{\infty} \leq n\|\nabla \phi\|_{\infty}$, and $\lim _{m \rightarrow \infty} I\left(\phi_{m}, p\right)=$ $I(\phi, p)$ whenever $1<p<\infty$. Moreover, $I\left(\phi_{m}, \cdot\right) \rightarrow I(\phi, \cdot)$ as $m \rightarrow \infty$, uniformly (in $p$ ) when $p \in[3 / 2,5 / 2]$.

Proof of Proposition 6.4. First, as pointed out in Remark 3.11, the constants in Lemmas $3.2-3.5$ can be chosen independent of $p$ when $p \in[3 / 2,5 / 2]$. Also the barrier argument in (6.2), as well as the subsequent Schwarz reflection argument, yield constants that are independent of $p$ when $p \in[3 / 2,5 / 2]$. Using these facts and retracing our steps we get (6.10) for $x_{n}-u^{\prime}(x, p)$ when $x \in \Omega^{\prime} \backslash \bar{B}(0,8 n)$ with constants that are independent of $p$ when $p \in[3 / 2,5 / 2]$.

Next we note that $(6.11)$ holds for $u^{\prime}(\cdot, p)$ on $\Omega^{\prime}$ with $\eta$ independent of $p$ when $p \in$ $[3 / 2,5 / 2]$. From this we deduce that $(6.14)$ holds on $\Omega^{\prime} \backslash \bar{B}(0,8 n)$ with $U$ replaced $x_{n}-u^{\prime}(x, p)$ (and with constants independent of $p$ when $p \in[3 / 2,5 / 2]$ ). We also observe that a check of the proof in [Li], mentioned after (6.16), yields the existence of $\gamma^{\prime}=\gamma^{\prime}(n)$ such that $u^{\prime}(\cdot, p) \in C^{1, \gamma^{\prime}}\left(\bar{\Omega}^{\prime} \cap \bar{B}(0,4 \rho)\right)$ whenever $p \in[3 / 2,5 / 2]$ and $\rho>0$, and with $C^{1, \gamma^{\prime}}$ norm depending only on $n, \phi, \rho$. Using this observation and (6.11) one sees that Schauder estimates can be used once again to get $u^{\prime} \in C^{\infty}\left(\bar{\Omega}^{\prime} \cap \bar{B}(0,2 \rho)\right)$, with $C^{3}\left(\bar{\Omega}^{\prime} \cap \bar{B}(0,2 \rho)\right)$ norm independent of $p$ when $p \in[3 / 2,5 / 2]$. Hence $u^{\prime}(\cdot, p) \rightarrow$ $u^{\prime}(\cdot, 2)$ in $C^{3}\left(\bar{\Omega}^{\prime} \cap \bar{B}(0, \rho)\right)$ for each $\rho>0$ as $p \rightarrow 2$. This deduction, (6.11), and (6.14) imply that $I(\phi, p) \rightarrow I(\phi, 2)$ as $p \rightarrow 2$. Thus Proposition 6.4 is true. 
Proof of Proposition 6.5. To define $\phi_{m}, m=1,2, \ldots$, we first take a grid of closed cubes of sidelength $\eta_{m}$ in $\mathbb{R}^{n-1}$ with sides parallel to the coordinate axes, and then divide each cube into closed simplexes with disjoint interiors. Next define $\phi_{m}$ to be the unique piecewise linear function on $\mathbb{R}^{n-1}$ that agrees with $\phi$ at the vertices of each simplex and is linear inside each simplex. Choosing $0<\eta_{m} \leq 10^{-20}, m=1,2, \ldots$, with $\eta_{m} \rightarrow 0$ as $m \rightarrow \infty$, we deduce from $\phi \in C_{0}^{\infty}\left(\left\{x^{\prime}:\left|x^{\prime}\right|<1 / 2\right\}\right)$ that $\left(\phi_{m}\right)$ is a sequence of piecewise linear functions satisfying

$$
\begin{aligned}
& \left\|\phi_{m}\right\|_{\infty} \leq\|\phi\|_{\infty} \quad \text { and } \quad \operatorname{supp} \phi_{m} \subset\left\{x^{\prime}:\left|x^{\prime}\right|<1 / 2\right\} \\
& \left\|\nabla \phi_{m}\right\|_{\infty} \leq n\|\nabla \phi\|_{\infty}, \\
& \lim _{m \rightarrow \infty}\left(\left\|\phi_{m}-\phi\right\|_{\infty}+\left\|\nabla \phi_{m}-\nabla \phi\right\|_{\infty}\right)=0
\end{aligned}
$$

For fixed $p \in(1, \infty)$, let $u_{m}^{\prime}, m=1,2, \ldots$, be the $p$-harmonic function in Lemma 6.1 defined relative to $\Omega_{m}^{\prime}=\left\{x=\left(x^{\prime}, x_{n}\right): x_{n}>\phi_{m}\left(x^{\prime}\right)\right\}$ and let $\mu_{m}^{\prime}, \mu^{\prime}$ be the measures corresponding to $u_{m}^{\prime}, u^{\prime}$ as in Lemma 3.6. From Lemmas 3.2-3.6 and Lemma 6.1 we deduce that

$$
\mu_{m}^{\prime} \rightarrow \mu^{\prime} \quad \text { weakly as } m \rightarrow \infty \text {. }
$$

From Theorem 3.10 we find that if $f_{m}(x)=\left|\nabla u_{m}^{\prime}\right|^{p-1}(x), x \in \partial \Omega_{m}^{\prime}, m=1,2, \ldots$, while $f(x)=\left|\nabla u^{\prime}\right|^{p-1}(x), x \in \partial \Omega^{\prime}$, then these functions exist $H^{n-1}$-almost everywhere and

$$
d \mu_{m}^{\prime}=f_{m} d H^{n-1} \quad \text { and } \quad d \mu^{\prime}=f d H^{n-1} .
$$

Let $\tilde{f}_{m}\left(x^{\prime}\right)=f_{m}\left(x^{\prime}, \phi_{m}\left(x^{\prime}\right)\right), m=1,2, \ldots$ Put $\tilde{f}\left(x^{\prime}\right)=f\left(x^{\prime}, \phi\left(x^{\prime}\right)\right)$ for $x^{\prime} \in \mathbb{R}^{n-1}$. Then

$$
\begin{gathered}
d \mu_{m}^{\prime}\left(x^{\prime}, \phi_{m}\left(x^{\prime}\right)\right)=\tilde{f}_{m}\left(x^{\prime}\right) \sqrt{1+\left|\nabla \phi_{m}\left(x^{\prime}\right)\right|^{2}} d x^{\prime}, \\
d \mu^{\prime}\left(x^{\prime}, \phi\left(x^{\prime}\right)\right)=\tilde{f}\left(x^{\prime}\right) \sqrt{1+\left|\nabla \phi\left(x^{\prime}\right)\right|^{2}} d x^{\prime}
\end{gathered}
$$

almost everywhere, with respect to $(n-1)$-dimensional Lebesgue measure on $\mathbb{R}^{n-1}$. Fix $\rho>n$ and let $B^{\prime}(0, \rho)=\left\{x^{\prime} \in \mathbb{R}^{n-1}:\left|x^{\prime}\right|<\rho\right\}$. From Theorem 3.10 we get the existence of $c(\rho)=c(\rho, p, n) \geq 1$ such that if $q=p /(p-1)$, then

$$
\left\|\tilde{f}_{m}\right\|_{L^{q}\left(B^{\prime}(0,2 \rho)\right)}+\|\tilde{f}\|_{L^{q}\left(B^{\prime}(0,2 \rho)\right)} \leq c(\rho) \quad \text { for } m=1,2, \ldots
$$

Moreover, $c(\rho)$ can be chosen independent of $p$ when $p \in[3 / 2,5 / 2]$. From (6.40)-(6.43) we conclude that

$$
\tilde{f}_{m} \rightarrow \tilde{f} \quad \text { weakly in } L^{q}\left(B^{\prime}(0,2 \rho)\right)
$$

for each $\rho>0$.

Next we state the following important lemma.

Lemma 6.6. $\tilde{f}_{m}-\tilde{f} \rightarrow 0$ in the norm of $L^{q}\left(\mathbb{R}^{n-1}\right)$, and this convergence is uniform (in $p$ ) when $p \in[3 / 2,5 / 2]$. 
Before giving the proof of Lemma 6.6, we use it to complete the proof of Proposition 6.5. To proceed we observe that from the smoothness of $\partial \Omega^{\prime}, u^{\prime}$, barrier estimates, and (6.14) there exists $\xi \in(0,1 / 10)$ with $\left|\nabla u^{\prime}\right| \geq 2 \xi$ on $\partial \Omega^{\prime}$. Furthermore, $\xi$ can be chosen independent of $p$ when $p \in[3 / 2,5 / 2]$. Let $E_{m}=\left\{x^{\prime} \in \mathbb{R}^{n-1}:\left|\tilde{f}_{m}\left(x^{\prime}\right)\right| \geq \xi\right\}$. From this observation, Lemma 6.6, and weak type estimates we see that

$$
H^{n-1}\left(\mathbb{R}^{n-1} \backslash E_{m}\right) \rightarrow 0 \quad \text { as } m \rightarrow \infty \text {, uniformly (in } p \text { ) when } p \in[3 / 2,5 / 2] \text {. }
$$

Observe, for $m=1,2, \ldots$, that

$$
\begin{aligned}
& I\left(\phi_{m}, p\right)=(p-1)^{-1} \int_{\mathbb{R}^{n-1}}\left|\tilde{f}_{m}\right|\left(x^{\prime}\right) \log \left|\tilde{f}_{m}\left(x^{\prime}\right)\right| \sqrt{1+\left|\nabla \phi_{m}\left(x^{\prime}\right)\right|^{2}} d x^{\prime} \\
& \quad=(p-1)^{-1} \int_{B^{\prime}(0, \rho)} \ldots d x^{\prime}+(p-1)^{-1} \int_{\mathbb{R}^{n-1} \backslash B^{\prime}(0, \rho)} \ldots d x^{\prime}=: J_{m}+K_{m} .
\end{aligned}
$$

We also write $I(\phi, p)=J+K$, where $J, K$ are defined as in (6.46) but with $\tilde{f}_{m}$ replaced by $\tilde{f}$. Given $\delta^{\prime}>0$ we deduce from (6.14) that there exists $\rho$ large so that

$$
\left|K_{m}\right|+|K|<\delta^{\prime} \quad \text { for } m=1,2, \ldots
$$

To estimate $J_{m}$ we write $J_{m}=N_{m}+S_{m}$ and $J=N_{m}^{\prime}+S_{m}^{\prime}$, where

$$
\begin{aligned}
& N_{m}=(p-1)^{-1} \int_{E_{m} \cap B^{\prime}(0, \rho)}\left|\tilde{f}_{m}\left(x^{\prime}\right)\right| \log \left|\tilde{f}_{m}\left(x^{\prime}\right)\right| \sqrt{1+\left|\nabla \phi_{m}\left(x^{\prime}\right)\right|^{2}} d x^{\prime}, \\
& S_{m}=(p-1)^{-1} \int_{B^{\prime}(0, \rho) \backslash E_{m}}\left|\tilde{f}_{m}\left(x^{\prime}\right)\right| \log \left|\tilde{f}_{m}\left(x^{\prime}\right)\right| \sqrt{1+\left|\nabla \phi_{m}\left(x^{\prime}\right)\right|^{2}} d x^{\prime}, \\
& N_{m}^{\prime}=(p-1)^{-1} \int_{E_{m} \cap B^{\prime}(0, \rho)}\left|\tilde{f}\left(x^{\prime}\right)\right| \log \left|\tilde{f}\left(x^{\prime}\right)\right| \sqrt{1+\left|\nabla \phi\left(x^{\prime}\right)\right|^{2}} d x^{\prime}, \\
& S_{m}^{\prime}=(p-1)^{-1} \int_{B^{\prime}(0, \rho) \backslash E_{m}}\left|\tilde{f}\left(x^{\prime}\right)\right| \log \left|\tilde{f}\left(x^{\prime}\right)\right| \sqrt{1+\left|\nabla \phi\left(x^{\prime}\right)\right|^{2}} d x^{\prime} .
\end{aligned}
$$

Using (6.45), the inequality

$$
t|\log t| \leq c\left(1+t^{(1+q) / 2}\right) \quad \text { on }(0, \infty)
$$

as well as Hölder's inequality and (6.43), we see that

$$
\left|S_{m}\right|+\left|S_{m}^{\prime}\right| \rightarrow 0 \quad \text { as } m \rightarrow \infty \text {, uniformly (in } p \text { ) when } p \in[3 / 2,5 / 2] .
$$

Finally using

$$
t|\log (t / s)|+|s-t||\log s| \leq c(\xi)|t-s|\left(t^{q-1}+s^{q-1}\right) \quad \text { for } s, t \in[\xi, \infty),
$$

as well as Hölder's inequality, (6.40), (6.43), and Lemma 6.6 we also find that

$$
\left|N_{m}-N_{m}^{\prime}\right| \rightarrow 0 \quad \text { as } m \rightarrow \infty \text {, uniformly (in } p \text { ) when } p \in[3 / 2,5 / 2] \text {. }
$$


From (6.46)-(6.50) we conclude that there exists a positive integer $m_{0}=m_{0}\left(\delta^{\prime}, \rho, p\right)$ such that

$$
\left|I\left(\phi_{m}, p\right)-I(\phi, p)\right|<2 \delta^{\prime} \quad \text { for } m \geq m_{0} .
$$

Moreover, $m_{0}$ can be chosen independent of $p$ when $p \in[3 / 2,5 / 2]$. Hence, as $\delta^{\prime}$ is arbitrary the proof of Proposition 6.5 is complete.

The rest of the section is devoted to the proof of Lemma 6.6. We will reuse the notation developed up to the statement of the lemma. Let $\psi^{*} \in C_{0}^{\infty}(B(0,2 \rho)), \psi^{*} \equiv 1$ on $B(0, \rho)$, and $\left|\nabla \psi^{*}\right| \leq c / \rho$. Put $\tilde{\psi}\left(x^{\prime}\right)=\psi^{*}\left(x^{\prime}, \phi\left(x^{\prime}\right)\right)$ when $x^{\prime} \in \mathbb{R}^{n-1}$. We first prove the following auxiliary lemma.

\section{Lemma 6.7.}

$$
\int \tilde{\psi} \tilde{f}_{m}(\cdot, p) \tilde{f}^{q-1}(\cdot, p) d x^{\prime} \rightarrow \int \tilde{\psi} \tilde{f}^{q}(\cdot, p) d x^{\prime}
$$

as $m \rightarrow \infty$, uniformly (in $p$ ) when $p \in[3 / 2,5 / 2]$.

Proof. Recall that $u_{m}^{\prime}, u^{\prime}$ are continuous on $\mathbb{R}^{n}$ with $u_{m}^{\prime}, u^{\prime} \equiv 0$ outside of $\Omega_{m}^{\prime}, \Omega^{\prime}$, respectively. We claim that

$$
\left.u_{m}^{\prime}(\cdot, p) \rightarrow u^{\prime}(\cdot, p) \quad \text { in } W^{1, p}(B(0,4 \rho)) \text {, uniformly (in } p\right) \text { when } p \in[3 / 2,5 / 2] .
$$

To prove this observe, from (6.40), Lemma 3.3, (6.10) with $\hat{u}$ replaced by $u^{\prime}, u_{m}^{\prime}$ and the maximum principle for $p$-harmonic functions, that

$$
\left\|u_{m}^{\prime}(\cdot, p)-u^{\prime}(\cdot, p)\right\|_{\infty} \rightarrow 0 \quad \text { as } m \rightarrow \infty \text {, uniformly (in } p \text { ) when } p \in[3 / 2,5 / 2] .
$$

Let $0 \leq \psi \in C_{0}^{\infty}(B(0,8 \rho))$ with $\psi \equiv 1$ on $B(0,4 \rho)$ and $\|\nabla \psi\|_{\infty} \leq c / \rho$. Using (6.53) and $\theta=\psi\left(u_{m}^{\prime}-u^{\prime}\right)$ as a test function in (1.3) with $u$ replaced by $u^{\prime}, u_{m}^{\prime}$, respectively, as well as Lemma 3.6(ii), we find that

$$
\int_{B(0,4 \rho)}\left(\left|\nabla u_{m}^{\prime}\right|^{p-2} \nabla u_{m}^{\prime}-\left|\nabla u^{\prime}\right|^{p-2} \nabla u^{\prime}\right) \cdot \nabla\left(u_{m}^{\prime}(\cdot, p)-u^{\prime}(\cdot, p)\right) d x \rightarrow 0
$$

as $m \rightarrow \infty$, uniformly (in $p$ ) when $p \in[3 / 2,5 / 2]$. The integral in (6.54) can be estimated from below by

$$
c^{-1} \int_{B(0,4 \rho)}\left(\left|\nabla u_{m}^{\prime}\right|+\left|\nabla u^{\prime}\right|\right)^{p-2}\left|\nabla u_{m}^{\prime}-\nabla u^{\prime}\right|^{2} d x .
$$

Moreover $\left\|\nabla u^{\prime}-\nabla u_{m}^{\prime}\right\|_{L^{p}(B(0,4 \rho))}$ can be estimated using the integral in (6.55). Doing this and using (6.54) we get (6.52).

To continue the proof of Lemma 6.7, we note from our discussion in the proof of Proposition 6.4 that $\left|\nabla u^{\prime}\right|$ has a $C^{2}$ extension, say $F(\cdot, p)$, to $B(0,4 \rho)$ with $C^{2}$ norm 
bounded by the $C^{2}$ norm of $\left|\nabla u^{\prime}\right|$ on $\Omega^{\prime} \cap B(0,4 \rho)$, uniformly (in $p$ ) when $p \in[3 / 2,5 / 2]$. We next put $\theta=\psi^{*} F$ in (1.3) with $\hat{u}$ replaced by $u^{\prime}, u_{m}^{\prime}$. We then get

$$
\begin{aligned}
& \left.\left|\int_{\partial \Omega^{\prime}} F \psi^{*}\right| \nabla u^{\prime}\right|^{p-1} d H^{n-1}-\int_{\partial \Omega_{m}^{\prime}} F \psi^{*}\left|\nabla u_{m}^{\prime}\right|^{p-1} d H^{n-1} \mid \\
& \quad=\left|\int_{B(0,1)}\left(\left|\nabla u^{\prime}\right|^{p-2} \nabla u^{\prime}-\left|\nabla u_{m}^{\prime}\right|^{p-2} \nabla u_{m}^{\prime}\right) \cdot \nabla\left(F \psi^{*}\right)\right| \\
& \quad \leq A\left(1+\left\|\left|\nabla u^{\prime}\right|\right\|_{L^{p}(B(0,2 \rho)}^{p-1}+\left\|\left|\nabla u_{m}^{\prime}\right|\right\|_{L^{p}(B(0,2 \rho)}^{p-1}\right)\left\|\nabla u_{m}^{\prime}-\nabla u^{\prime}\right\|_{L^{p}(B(0, \rho))}^{\xi} .
\end{aligned}
$$

In (6.56), $\xi=\min (p-1,1)$, while $A$ depends on $\rho$ and the $C^{1}$ norm of $F$ but can be chosen independent of $p$ when $p \in[3 / 2,5 / 2]$. In particular, using (6.56), (6.52) and Hölder's inequality we can conclude that

$$
\left.\left|\int_{\partial \Omega^{\prime}} F \psi^{*}\right| \nabla u^{\prime}\right|^{p-1} d H^{n-1}-\int_{\partial \Omega_{m}^{\prime}} F \psi^{*}\left|\nabla u_{m}^{\prime}\right|^{p-1} d H^{n-1} \mid \rightarrow 0 \quad \text { as } m \rightarrow \infty .
$$

Rewriting the left-hand side of (6.57) in terms of $\tilde{f}_{m}, \tilde{f}$, using (6.40) and smoothness of $F$, we deduce that Lemma 6.7 is true.

Proof of Lemma 6.6. To prove Lemma 6.6 we use a Rellich type inequality and argue as in displays (2.40)-(2.42) and (5.30)-(5.35) of [LN1]. Let $\hat{C}$ be the cone obtained by drawing rays from $\rho e_{n}$ through points in $\left(\mathbb{R}^{n-1} \times\{0\}\right) \cap B^{\prime}(0, \rho)$ to $\infty$. First for fixed $m$, $u_{m}^{\prime}$ as above, and given $\eta>0$ small, we apply the divergence theorem to the vector field $\left|\nabla u_{m}^{\prime}\right|^{p} e_{n}$ in $D_{m}=\left\{y \in \Omega_{m}^{\prime}: y_{n}>\phi_{m}\left(y^{\prime}\right)+\eta\right\} \cap \hat{C}$. We get

$$
\begin{aligned}
\int_{\partial D_{m}}\left\langle e_{n}, v_{m}\right\rangle\left|\nabla u_{m}^{\prime}\right|^{p} d H^{n-1} & =\int_{D_{m}} \nabla \cdot\left(e_{n}\left|\nabla u_{m}^{\prime}\right|^{p}\right) d x \\
& =p \sum_{i=1}^{n} \int_{D_{m}}\left|\nabla u_{m}^{\prime}\right|^{p-2}\left(u_{m}^{\prime}\right)_{x_{i}}\left(u_{m}^{\prime}\right)_{x_{i} x_{n}} d x \\
& =p \int_{\partial D_{m}}\left|\nabla u_{m}^{\prime}\right|^{p-2}\left\langle\nabla u_{m}^{\prime}, v_{m}\right\rangle\left(u_{m}^{\prime}\right)_{x_{n}} d H^{n-1},
\end{aligned}
$$

where $v_{m}$ denotes the outer unit normal to $D_{m}$. In obtaining the last equality we have used integration by parts and $p$-harmonicity of $u_{m}^{\prime}$. Letting $\eta \rightarrow 0$ in (6.58), using Theorem 3.10(i), (iv), as well as the Lebesgue dominated convergence theorem, we find that

$$
\int_{\partial D_{m}^{\prime}}\left[\left\langle e_{n}, v_{m}^{\prime}\right\rangle\left|\nabla u_{m}^{\prime}\right|^{p}-p\left|\nabla u_{m}^{\prime}\right|^{p-2}\left\langle\nabla u_{m}^{\prime}, v_{m}^{\prime}\right\rangle\left(u_{m}^{\prime}\right)_{x_{n}}\right] d H^{n-1}=0,
$$

where $v_{m}^{\prime}$ denotes the outer unit normal to $D_{m}^{\prime}=\Omega_{m}^{\prime} \cap \hat{C}$. (6.59) also holds with $D_{m}^{\prime}, u_{m}^{\prime}, v_{m}^{\prime}$ replaced by $D^{\prime}, u^{\prime}, v^{\prime}$, where $D^{\prime}=\Omega^{\prime} \cap \hat{C}$ and $v^{\prime}$ is the outer unit normal to $D^{\prime}$. From Theorem 3.10(i) we also get

$$
\int_{\partial D_{m}^{\prime}}\left|\nabla u_{m}^{\prime}\right|^{r} d H^{n-1} \leq c<\infty, \quad \text { where } r / p \geq 1+\delta .
$$


Here $0<\delta=\delta(p, n)$ is uniformly (in $p$ ) bounded from below when $p \in[3 / 2,5 / 2]$. Moreover, we note from Lemmas 3.2-3.5, (6.40), (6.52), and Lemma 6.1 that

$$
\nabla u_{m}^{\prime} \rightarrow \nabla u^{\prime} \quad \text { uniformly on compact subsets of } \Omega^{\prime} \text { as } m \rightarrow \infty \text {. }
$$

Moreover for a given compact set the convergence is also uniform (in $p$ ) when $p \in$ [3/2, 5/2]. Using (6.61), (6.40), (6.60), and uniform integrability type arguments we deduce that

$$
\begin{aligned}
0 & =\int_{\partial D_{m}^{\prime} \cap \Omega^{\prime}}\left[\left\langle e_{n}, v_{m}^{\prime}\right\rangle\left|\nabla u_{m}^{\prime}\right|^{p}-p\left|\nabla u_{m}^{\prime}\right|^{p-2}\left\langle\nabla u_{m}^{\prime}, v_{m}^{\prime}\right\rangle\left(u_{m}^{\prime}\right)_{x_{n}}\right] d H^{n-1} \\
& \rightarrow \int_{\partial D^{\prime} \cap \Omega^{\prime}}\left[\left\langle e_{n}, v^{\prime}\right\rangle\left|\nabla u^{\prime}\right|^{p}-p\left|\nabla u^{\prime}\right|^{p-2}\left\langle\nabla u^{\prime}, v^{\prime}\right\rangle u_{x_{n}}^{\prime}\right] d H^{n-1}
\end{aligned}
$$

as $m \rightarrow \infty$, uniformly (in $p$ ) when $p \in[3 / 2,5 / 2]$. In view of (6.59) for $u_{m}^{\prime}, u^{\prime},(6.62)$, and the fact that $-v^{\prime}=\nabla u^{\prime} /\left|\nabla u^{\prime}\right|,-v_{m}^{\prime}=\nabla u_{m}^{\prime} /\left|\nabla u_{m}^{\prime}\right|$, on $\partial \Omega^{\prime}, \partial \Omega_{m}^{\prime}$, respectively, we have

$$
\int_{\partial D_{m}^{\prime} \cap \partial \Omega^{\prime}}\left\langle e_{n}, v_{m}^{\prime}\right\rangle\left|\nabla u_{m}^{\prime}\right|^{p} d H^{n-1} \rightarrow \int_{\partial D^{\prime} \cap \partial \Omega^{\prime}}\left\langle e_{n}, v^{\prime}\right\rangle\left|\nabla u^{\prime}\right|^{p} d H^{n-1}
$$

as $m \rightarrow \infty$, uniformly (in $p$ ) when $p \in[3 / 2,5 / 2]$. Writing (6.63) with integrals over $B^{\prime}(0, \rho)$ and recalling the definition of $\tilde{f}_{m}, \tilde{f}$, we see that

$$
\int_{B^{\prime}(0, \rho)} \tilde{f}_{m}^{q} d H^{n-1} \rightarrow \int_{B^{\prime}(0, \rho)} \tilde{f}^{q} d H^{n-1}
$$

as $m \rightarrow \infty$, uniformly (in $p$ ) when $p \in[3 / 2,5 / 2]$.

To conclude the proof of Lemma 6.6 we use (6.64), Lemma 6.7, and with minor changes essentially copy the usual proof that weak and norm convergence imply strong convergence in $L^{q}\left(\mathbb{R}^{n-1}\right)$ (see [RN, p. 78]). In the following we only give the details of the proof in the case when $q \geq 2$. Indeed, given $\delta^{\prime}>0$ we can use (6.14) to find $\rho>0$ large so that

$$
\int_{\mathbb{R}^{n} \backslash B^{\prime}(0, \rho)}\left(\tilde{\psi}\left|\tilde{f}_{m}-\tilde{f}\right| \tilde{f}^{q-1}+\left|\tilde{f}_{m}-\tilde{f}\right|^{q}+\left|\tilde{f}^{q}-\tilde{f}_{m}^{q}\right|\right) d x^{\prime} \leq \delta^{\prime}
$$

whenever $m=1,2, \ldots$ Moreover, it follows from (6.64) and Lemma 6.7 that we can choose $m_{0}$ large enough (independent of $p$ when $p \in[3 / 2,5 / 2]$ ) so that

$$
\left|\int_{B^{\prime}(0, \rho)}\left(\tilde{f}_{m}-\tilde{f}\right) \tilde{f}^{q-1} d x^{\prime}\right|+\left|\int_{B^{\prime}(0, \rho)}\left(\tilde{f}^{q}-\tilde{f}_{m}^{q}\right) d x^{\prime}\right| \leq 2 \delta^{\prime} \quad \text { for } m \geq m_{0} .
$$

From these inequalities we conclude, for some absolute $c \geq 1$ and given $\epsilon>0$, that

$$
c^{-1} \int_{\mathbb{R}^{n-1}}\left|\tilde{f}_{m}-\tilde{f}\right|^{q} d x^{\prime} \leq\left|\int_{\mathbb{R}^{n-1}}\left[\tilde{f}_{m}^{q}-\tilde{f}^{q}-q \tilde{f}^{q-1}\left(\tilde{f}_{m}-\tilde{f}\right)\right] d x^{\prime}\right| \leq \epsilon
$$

for $m \geq m_{0}$ provided $\delta^{\prime}$ is small enough. This concludes the proof of Lemma 6.6. 
Remark 6.8. Wolff proves Proposition 6.5 using a Rellich inequality for $u_{m}^{\prime}-u^{\prime}$. However, the proof of this inequality uses linearity of Laplace's equation. Thus we were forced to give a different argument when $1<p<\infty, p \neq 2$. Eventually we arrived at the above argument.

\section{Estimates of Wolff type integrals-the case of an unbounded Reifenberg flat domain}

\subsection{An ergodic argument for the dimension of p-harmonic measure and the proof of} Theorems 2-4

We now return to the construction of a Wolff snowflake outlined in Section 2; in this section we use ideas of Carleson [C] to reduce the proof of Theorems 2 and 4 to the estimation of integrals of the form $I$ alluded to in Section 2. As in Section 2, let $\phi$ : $\mathbb{R}^{n-1} \rightarrow \mathbb{R}$ be a piecewise linear function with

$$
\|\nabla \phi\|_{\infty} \leq \theta_{0}
$$

and with support contained in $\left\{x^{\prime}:\left|x^{\prime}\right|<1 / 2\right\}$. Let $\psi\left(x^{\prime}\right)=N^{-1} \phi\left(N x^{\prime}\right), x^{\prime} \in \mathbb{R}^{n-1}$, for fixed $N \geq N_{0}$, and define $\Lambda$, $\partial$ relative to $\psi$ as in (2.12). Here $\theta_{0}, N_{0}^{-1}$ are small positive numbers depending on $p, n$, but it turns out that they can be chosen independent of $p$ when $p \in[3 / 2,5 / 2]$. Given an $n-1$-cube $Q$ recall the definition of $P_{Q}, \tilde{P}_{Q}$ in (2.11) defined relative to $b=1 / 10$ and a unit normal $e$. Next given a region $\Omega$ with $Q \subset \partial \Omega$, let $e$ be the outer unit normal to $\partial \Omega$ on $Q$ and suppose that $P_{Q} \cap \Omega=\emptyset$ while $\tilde{P}_{Q} \subset \Omega$. With this scenario recall the definition of adding a blip to $\Omega$ along $Q$ defined below (2.12). That is, we form a new domain $\tilde{\Omega}$ with $\left(P_{Q} \cup \tilde{P}_{Q}\right) \cap \tilde{\Omega}=\Lambda_{Q}$ and $\left(P_{Q} \cup \tilde{P}_{Q}\right) \cap \partial \tilde{\Omega}=\partial_{Q}$, while $\Omega=\tilde{\Omega}$ outside of $P_{Q} \cup \tilde{P}_{Q}$. Here $T(\Lambda)=\Lambda_{Q}, T(\partial)=\partial_{Q}$, where $T$ is the conformal affine mapping defined below (2.12). $\Lambda_{Q}$ is the blip added to $\Omega$ along $Q$. In Section 2 we added a blip to $\Omega_{0}=\left\{\left(x^{\prime}, x_{n}\right): x^{\prime} \in \mathbb{R}^{n-1}, x_{n}>0\right\}$ along $Q(1)$ to obtain $\Omega_{1}$. Moreover, each face of $\partial=\partial_{Q(1)}$ was divided into $n-1$-dimensional Whitney cubes of sidelengths $8^{-k}, k=1,2, \ldots$, which are proportional to the distance of the cubes from the edges of the face that contains the cube. Notice that we added a blip along a cube of sidelength 1 and that the generated Whitney cubes all have strictly smaller sidelengths. We also put $G_{1}=\left\{Q: Q \subset \partial_{Q(1)}\right\}$ and let $\Omega_{2}$ be the domain obtained from $\Omega_{1}$ by adding a blip to each $Q \in G_{1}$. Next we divided $\partial_{Q}, Q \in G_{1}$, into cubes using the natural subdivision obtained from mapping $\partial_{Q(1)}$ conformally onto $\partial_{Q}$. Put $G_{2}=\left\{Q: Q \subset \partial_{Q^{\prime}}\right.$ for some $\left.Q^{\prime} \in G_{1}\right\}$ and note that

$$
\partial \Omega_{i} \cap\left(P_{Q(1)} \cup \tilde{P}_{Q(1)}\right)=E_{i} \cup\left\{Q: Q \in G_{i}\right\} \quad \text { for } i=1,2,
$$

where $E_{1}$ is the finite set of edges of $\partial=\partial_{Q(1)}$ and $E_{2}$ is a countable union of edges. Proceeding by induction we get, as in Section $2,\left(\Omega_{m}\right),\left(G_{m}\right)$, and $\left(E_{m}\right)$ with

$$
\partial \Omega_{m} \cap\left(P_{Q(1)} \cup \tilde{P}_{Q(1)}\right)=E_{m} \cup\left\{Q: Q \in G_{m}\right\}
$$


for $m=1,2, \ldots$ Let $\Omega_{\infty}=\bigcap_{m=1}^{\infty} \bigcup_{i=m}^{\infty} \Omega_{i}$. It is easily seen for $\theta_{0}, N_{0}^{-1}$ small enough that $h\left(\Omega_{m}, \Omega_{\infty}\right) \rightarrow 0$ as $m \rightarrow \infty$ where $h(\cdot, \cdot)$ denotes Hausdorff distance defined as in Section 1 .

We now put $G_{0}=\{Q(1)\}$ and set $G=\bigcup G_{m}$ and $E=\bigcup E_{m}$. As in [W2], we say that $Q \in G$ is the father of $Q^{\prime} \in G$ or $Q^{\prime}$ is the son of $Q$ if $Q^{\prime} \subset \partial_{Q}$. Moreover, $Q^{\prime}$ is an $m$ th generation descendant of $Q$ provided there exist $Q_{1}, \ldots, Q_{m+1}$ with $Q_{1}=Q$, $Q_{m+1}=Q^{\prime}$ and $Q_{i}$ the father of $Q_{i+1}$ for $i=1, \ldots, m$. We write $Q^{\prime}<Q$ provided $Q^{\prime}$ is for some $m$ a descendant of $Q$.

Given nonempty $\Gamma \subset G$ we say that $\Gamma$ is closed under the descent relation if whenever $Q^{\prime} \in \Gamma$ and $Q^{\prime}<Q \in G$, then $Q \in \Gamma$. Thus for any $\Gamma$ closed under descent we always have $Q(1) \in \Gamma$. If $\Gamma$ is closed under the descent relation we can form a domain $\Omega_{\Gamma}$ by adding blips over cubes in $\Gamma$. That is, $\Omega_{\Gamma}^{2}$ is obtained from $\Omega_{1}$ by adding blips over the cubes in $G_{1} \cap \Gamma$ and for $m \geq 2, \Omega_{\Gamma}^{m+1}$ is obtained from $\Omega_{\Gamma}^{m}$ by adding blips over the cubes in $G_{m} \cap \Gamma$. Either the process terminates for some $m$ so that $\Omega_{\Gamma}^{m}=\Omega_{\Gamma}$, or $\Omega_{\Gamma}^{m} \rightarrow \Omega_{\Gamma}$ as $m \rightarrow \infty$ in the sense of Hausdorff distance provided $\theta_{0}, N_{0}^{-1}$ are small enough, depending only on $n$. Finally recall from Section 3 that $\Omega$ is Lipschitz on scale $t$ with norm $\leq M$ provided that for each $x \in \partial \Omega$ the set $B(x, t) \cap \partial \Omega$ is the graph of a Lipschitz function $f: \mathbb{R}^{n-1} \rightarrow \mathbb{R}$ with $\|\nabla f\|_{\infty} \leq M$. The next lemma shows that stages in the construction can be in the class of Reifenberg flat domains or even Lipschitz on scale $t$.

Lemma 7.1. Let $\Gamma \subset G$ be closed under the descent relation. For $\theta_{0}, N_{0}^{-1}$ small enough, depending only on $n$, there exists $c=c(n)$ so that

(a) $\Omega_{\Gamma}$ is Reifenberg flat with constant $\leq c \theta_{0}$.

(b) If $\Gamma$ contains only cubes of diameter $\geq t>0$, then $\Omega_{\Gamma}$ is Lipschitz on a scale $t$, with norm $\leq c \theta_{0}$.

Proof. Wolff essentially proves (b) in [W2, Lemma 2.2] and also shows that $\Omega_{\Gamma}$ is an NTA-domain. To prove (a) we assume as we may that $\Gamma \cap G_{m}=\emptyset$ for some large $m$. Given $r>0$ and $x \in \partial \Omega_{\Gamma}$ we claim there exists a plane $\Sigma=\Sigma(x, r)$ and $c=c(n)$ such that

$$
h\left(\Sigma \cap B(x, r), \partial \Omega_{\Gamma} \cap B(x, r)\right) \leq c \theta_{0} .
$$

If $x \in \partial \Omega_{\Gamma} \backslash\left(P_{Q(1)} \cup \tilde{P}_{Q(1)}\right)$, we choose $\Sigma=\left\{x: x_{n}=0\right\}$ and observe that (7.2) is true, as follows easily from (7.1) with $\phi$ replaced by $\psi$, the fact that $\psi$ has support in $Q(1 / N)$, and the construction of $\Omega_{\Gamma}$. Also if $x \in \partial \Omega_{\Gamma} \cap\left(P_{Q(1)} \cup \tilde{P}_{Q(1)}\right)$ and $r \geq 1$, we put $\Sigma=\left\{x: x_{n}=0\right\}$ and observe that (7.2) is true. Otherwise, $r<1$ and if $x \notin E$, there exist $Q_{1}, \ldots, Q_{k} \in \Gamma, 2 \leq k \leq m+1$, with $Q_{1}=Q(1), x \in Q_{k}$, and $Q_{i}$ the father of $Q_{i+1}$ for $1 \leq i \leq k-1$. In this case we either have $(\alpha) l\left(Q_{i+1}\right) \leq r<l\left(Q_{i}\right)$ for some $i$, or $(\beta) r<l\left(Q_{k}\right)$. In case $(\alpha)$ we choose $\Sigma$ to be the plane through $x$ parallel to $Q_{i}$. Then from the definition of adding a blip we see, for $N_{0}$ large enough, that each point in $\Sigma \cap B(x, r)$ lies within $c \theta_{0} r$ of a point in $\partial \Omega_{\Gamma} \cap B(x, r)$ and vice versa. In case $(\beta)$ we let $\Sigma$ be the plane containing $Q_{k}$ and observe that once again (7.2) holds. If $x \in E$ choose $y \in \partial \Omega_{\Gamma} \backslash E$ with $|y-x|<\theta_{0} r$ and use the plane corresponding to $y, r$ to show that (7.2) holds. This completes the proof of Lemma 7.1. 
Given $p \in(1, \infty)$, observe from Lemma 7.1 that if $\theta_{0}, N_{0}^{-1}>0$ are small enough, $N_{0}=N_{0}(n), \theta_{0}=\theta_{0}(p, n)$, then the lemmas in Section 3 for Reifenberg flat domains hold with $\Omega$ replaced by $\Omega_{\Gamma}$. Furthermore, if $\Gamma$ contains no cubes of diameter $\leq r$, then Theorem 3.10 holds in balls of radius $\approx r$. Moreover, constants can be chosen independent of $p$ when $p \in[3 / 2,5 / 2]$. In the following we suppose that $\theta_{0}, N_{0}^{-1}$ are so small that all of the above hold. In general we will allow $N_{0}^{-1}, \theta_{0}$ to vary so that several more conditions are satisfied, however eventually these numbers will be fixed.

The next lemma is the crucial one for obtaining estimates over the stages of the construction.

Lemma 7.2. Let $\Gamma^{\prime} \subset \Gamma \subset G$, suppose that $\Gamma, \Gamma^{\prime}$ are closed under the descent relation and that $h\left(\partial \Omega_{\Gamma}, \partial \Omega_{\Gamma^{\prime}}\right)=r$. Given $p \in(1, \infty)$, let $u_{\Gamma}, u_{\Gamma^{\prime}}$ be the p-harmonic functions in Lemma 6.1 corresponding to $\Omega_{\Gamma}, \Omega_{\Gamma^{\prime}}$ and let $\mu_{\Gamma}, \mu_{\Gamma^{\prime}}$ be the associated measures. Let $\alpha=\alpha(p, n) \in(0,1)$ be as in Lemma 3.3. Then for $\theta_{0}, N_{0}^{-1}$ sufficiently small, there exists $c=c(p, n) \geq 1$ such that

$$
\text { (a) }\left|\log \frac{u_{\Gamma}(x)}{u_{\Gamma^{\prime}}(x)}\right| \leq\left(\frac{c r}{s}\right)^{\alpha}
$$

for all $x \in \Omega_{\Gamma^{\prime}}$ with $d\left(x, \partial \Omega_{\Gamma^{\prime}}\right) \geq s \geq 100 r$. Moreover if

$$
\left(\Omega_{\Gamma^{\prime}} \backslash \Omega_{\Gamma}\right) \cup\left(\Omega_{\Gamma} \backslash \Omega_{\Gamma^{\prime}}\right) \subset B\left(a_{\hat{Q}}, 4 l(\hat{Q}) / N\right)
$$

for some $\hat{Q} \in \Gamma^{\prime}$, then

$$
\text { (b) }\left|\log \frac{u_{\Gamma}(x)}{u_{\Gamma^{\prime}}(x)}\right| \leq\left(\frac{c r}{s}\right)^{\alpha} \frac{\mu_{\Gamma^{\prime}}\left(B\left(a_{\hat{Q}}, l(\hat{Q}) / N\right)\right)}{\mu_{\Gamma^{\prime}}\left(B\left(a_{\hat{Q}}, s\right)\right)}
$$

for all $x \in \Omega_{\Gamma^{\prime}}$ with $\left|x-a_{\hat{Q}}\right| \geq s \geq 100 l(\hat{Q}) / N$. In both of these estimates the constant $c$ can be chosen independent of $p$ when $p \in[3 / 2,5 / 2]$.

In Lemma 7.2, $a_{\hat{Q}}$ is the center of $\hat{Q}$ as defined in Section 2. For the moment we assume Lemma 7.2 in order to broadly outline the Carleson-Wolff game plan (see [C], [W2]), and to prove Theorems $2-4$. The proof of Lemma 7.2 will be given in the next subsection. For fixed $p \in(1, \infty)$, let $\Omega_{\infty}$ be the Wolff snowflake constructed earlier in Section 7 and let $u_{\infty}, \mu_{\infty}$ be the corresponding $p$-harmonic function and measure. Given $Q \in G$ let $\Theta_{Q}=\left(P_{Q} \cup \tilde{P}_{Q}\right) \cap \partial \Omega_{\infty}$ and let $E_{Q}$ be the union of the edges in $\partial_{Q^{\prime}}$ for all $Q^{\prime} \in G$ with $Q^{\prime} \subset Q$. Clearly, $E_{Q}$ is a countable union of edges and each edge has finite $H^{n-2}$ measure. If $Q \in G_{1}$ we can identify points in $\Theta_{Q} \backslash E_{Q}$ with points in $Q$, using sequences in a countable set of symbols (see [W2]). Doing this we can define the shift $S$ on $\Theta_{Q} \backslash E_{Q}$ by letting $\left.S\right|_{Q}$ be the inverse of the mapping $T$ defined in Section 2 relative to $Q$. Thus $\left.S\right|_{Q}$ is a conformal affine map of $Q$ onto $Q(1)$, so $S\left(\Theta_{Q}\right)=\Theta_{Q(1)}$ and $S$ is defined on $\Theta_{Q(1)}$ up to a set of $\sigma$-finite $H^{n-2}$-measure. As in Section 2 we let $\mu_{\infty}^{\prime}=\left.\mu_{\infty}\right|_{\Theta_{Q(1)}}$. Following [C], [W2] we prove

Lemma 7.3. $\mu_{\infty}^{\prime}$ is mutually absolutely continuous with respect to a measure $v$ on $\Theta_{Q(1)}$. Moreover $v$ is invariant under $S$ and $S$ is ergodic on $\Theta_{Q(1)}$. 
Proof. Let $F_{Q}(\epsilon)=\left\{x \in \partial \Omega_{\infty}: d(x, \partial Q) \leq \epsilon l(Q)\right\}$ whenever $Q \in G$ and $0<\epsilon<1$. Using the doubling property of $\mu_{\infty}$ (see Lemma 3.6(ii)) one deduces the existence of $\lambda=\lambda(p, n) \in(0,1)$ such that $\mu_{\infty}\left(F_{Q}(\epsilon)\right) \leq \lambda \mu_{\infty}\left(F_{Q}(2 \epsilon)\right)$. Iterating this inequality we find, for some $\beta>0$ and $c \geq 1$, depending only on $p, n$, that

$$
\mu_{\infty}\left(F_{Q}(\epsilon)\right) \leq c \epsilon^{\beta} \mu_{\infty}\left(\Theta_{Q}\right)
$$

A similar estimate holds for any of the edges in $\partial_{Q(1)}$. It follows that $\mu_{\infty}\left(E_{Q(1)}\right)=0$. Thus $S$ is defined $\mu_{\infty}^{\prime}$-almost everywhere on $\Theta_{Q(1)}$.

We now define $v$. For $Y \subset \Theta_{Q(1)}$ a Borel set, let

$$
v^{(m)}(Y)=\frac{1}{m} \sum_{j=0}^{m-1} \mu_{\infty}^{\prime}\left(S^{-j} Y\right)
$$

and let $v$ be a weak ${ }^{*}$ limit point of $\left\{v^{(m)}\right\}$. Note that here $S^{-0} Y=Y$. Furthermore,

$$
v^{(m)}\left(S^{-1} Y\right)-v^{(m)}(Y)=\frac{1}{m}\left(\mu_{\infty}^{\prime}\left(S^{-m} Y\right)-\mu_{\infty}^{\prime}(Y)\right) \rightarrow 0 \quad \text { as } m \rightarrow \infty,
$$

since $\mu_{\infty}^{\prime}$ is finite. Thus $v$ is invariant under $S$. To prove mutual absolute continuity, given $\epsilon>0$, one first shows, for some $c_{\epsilon}=c(\epsilon) \geq 1$, that

$$
\text { if } Y \subset \Theta_{Q(1)} \text { is Borel and } Y \cap F_{Q(1)}(\epsilon)=\emptyset \text {, then } c_{\epsilon}^{-1} \mu_{\infty}^{\prime}(Y) \leq v(Y) \leq c_{\epsilon} \mu_{\infty}^{\prime}(Y) \text {. }
$$

To prove (7.4) we use Lemma 3.6(ii), the boundary Harnack inequality in Theorem 3.9, as well as invariance of the $p$-Laplace equation under rotations, translations and dilations, to get for $Q \in G_{m}, m \geq 1$,

$$
c_{\epsilon}^{-1} \mu_{\infty}^{\prime}(Y) \mu_{\infty}^{\prime}\left(\Theta_{Q}\right) \leq \mu_{\infty}^{\prime}\left(S^{-m} Y \cap \Theta_{Q}\right) \leq c_{\epsilon} \mu_{\infty}^{\prime}(Y) \mu_{\infty}^{\prime}\left(\Theta_{Q}\right) .
$$

For more details concerning the deduction of (7.5), see [W2, Lemma 2.10] or [BL, (3.14)(3.16)]. Summing over $Q \in G_{m}$, and then $m$, we see that (7.4) holds. If $Y \cap F_{Q(1)}(\epsilon) \neq \emptyset$, we let $Y=Y_{1} \cup Y_{2}$ where $Y_{1}, Y_{2}$ are disjoint and $Y_{1}=Y \cap F_{Q(1)}(\epsilon)$. Then $S^{-m} Y_{1} \cap Q \subset$ $F_{Q}(\epsilon)$ whenever $Q \in G_{m}$. Using this fact, (7.3), and summing over $Q \in G_{m}$, as well as $m$, we obtain

$$
v\left(Y_{1}\right)+\mu_{\infty}^{\prime}\left(Y_{1}\right) \leq c \epsilon^{\beta} .
$$

In view of (7.5) and (7.4), we conclude that

$$
\mu_{\infty}^{\prime}(Y) \leq c_{\epsilon} \nu(Y)+c \epsilon^{\beta}
$$

and vice versa, whenever $Y$ is a Borel subset of $\Theta_{Q(1)}$. Since $\epsilon \in(0,1)$ is arbitrary it follows that $\mu_{\infty}^{\prime}, v$ are mutually absolutely continuous.

Having established the existence of $v$, the invariance of $v$ under $S$, and that $\mu_{\infty}^{\prime}$ is mutually absolutely continuous with respect to the measure $v$ on $\Theta_{Q(1)}$, it remains to establish that $S$ is ergodic on $\Theta_{Q(1)}$ with respect to $v$, that is, we need to show, whenever $H \subset \Theta_{Q(1)}$ is Borel and $S^{-1}(H)=H$, that either $\nu\left(\Theta_{Q(1)} \backslash H\right)=0$ or $v(H)=0$. 
To do this we will assume $v(H)>0$ and prove that $v\left(\Theta_{Q(1)} \backslash H\right)=0$. Set $L=$ $\Theta_{Q(1)} \backslash H$. For $x \in \Theta_{Q(1)} \backslash E_{Q(1)}$ and $m=1,2, \ldots$, let $Q_{m}(x)$ be the cube in $G_{m}$ with $x \in \Theta_{Q_{m}(x)}$. As $v(H)>0$, we choose $\epsilon>0$ so small that $v\left(F_{Q(1)}(\epsilon)\right)<v(H) / 4$, which can be done thanks to (7.6) with $Y_{1}=F_{Q(1)}(\epsilon)$. Let $H^{\prime}=H \backslash F_{Q(1)}(\epsilon)$ and suppose $x \in L \backslash F_{Q(1)}(2 \epsilon)$. Then for $m$ large enough, $\Theta_{Q_{m}(x)} \cap F_{Q(1)}(\epsilon)=\emptyset$. By (7.4), (7.5) with $Y=H^{\prime}, S^{-m} H^{\prime} \cap \Theta_{Q_{m}(x)}, \Theta_{Q_{m}(x)}$, we have

$$
\begin{aligned}
\frac{v\left(H \cap \Theta_{Q_{m}(x)}\right)}{v\left(\Theta_{Q_{m}(x)}\right)} & =\frac{v\left(S^{-m} H \cap \Theta_{Q_{m}(x)}\right)}{v\left(\Theta_{Q_{m}(x)}\right)} \\
& \geq \frac{v\left(S^{-m} H^{\prime} \cap \Theta_{Q_{m}(x)}\right)}{v\left(\Theta_{Q_{m}(x)}\right)} \geq c_{\epsilon}^{-3} v\left(H^{\prime}\right) \geq 3 c_{\epsilon}^{-3} v(H) / 4 .
\end{aligned}
$$

From the doubling property of $\mu_{\infty}^{\prime},(7.4)$, and differentiation theory, we deduce

$$
\frac{v\left(H \cap \Theta_{Q_{m}(x)}\right)}{v\left(\Theta_{Q_{m}(x)}\right)} \rightarrow 0
$$

for $\nu$-almost every $x \in L \backslash F_{Q(1)}(2 \epsilon)$ (i.e., $\nu$-almost every $x \in L \backslash F_{Q(1)}(2 \epsilon)$ is a point of $\nu$ density one). (7.7) and the above display are contradictory unless $v\left(L \backslash F_{Q(1)}(2 \epsilon)\right)=0$. Since $\epsilon$ can be arbitrarily small we conclude that $v(L)=0$. This completes the proof of Lemma 7.3.

Armed with Lemma 7.3 one can now apply appropriate versions of Birkhoff's ergodic theorem and the entropy theorem of Shannon-McMillan-Breiman to prove the following lemma (see [W2, Lemma 2.10]).

Lemma 7.4. Given $x \in \Theta_{Q(1)} \backslash E_{Q(1)}$, let $Q_{m}(x)$, for $m=1,2, \ldots$, be the cube in $G_{m}$ with $x \in \Theta_{Q_{m}(x)}$. Let $\sigma_{m}(x)=l\left(Q_{m}(x)\right)$ and $h_{m}(x)=\mu_{\infty}\left(\Theta_{Q_{m}(x)}\right)$. Then the limits

$$
\sigma=\lim _{m \rightarrow \infty} \frac{1}{m} \log \frac{1}{\sigma_{m}(x)} \quad \text { and } \quad h=\lim _{m \rightarrow \infty} \frac{1}{m} \log \frac{1}{h_{m}(x)}
$$

exist and are constant for $\nu$-almost every $x \in \Theta_{Q(1)}$.

This lemma gives us access to H-dim $\mu_{\infty}^{\prime}$ as follows. For $x \in \Theta_{Q(1)} \backslash E_{Q(1)}$ and $t \in(0,1)$ let $m$ be a nonnegative integer such that $\sigma_{m+1}(x) \leq t<\sigma_{m}(x)$. Note that $t \approx \sigma_{m}(x) \approx$ $\sigma_{m+1}(x)$ with constants of proportionality independent of $m$ and $x$. Using the doubling property of $\mu_{\infty}$, we see that

$$
\left|\log \frac{h_{m}(x)}{\mu_{\infty}^{\prime}(B(x, t))}\right| \leq c
$$

for some $c=c(p, n) \in[1, \infty)$. Using these facts, Lemma 7.4 and measure-theoretic type arguments it follows that

$$
\lim _{t \rightarrow 0} \frac{\log \mu_{\infty}^{\prime}(B(x, t))}{\log t}=\lim _{m \rightarrow \infty} \frac{\log h_{m}(x)}{\log \sigma_{m}(x)}=h / \sigma=\mathrm{H}-\operatorname{dim} \mu_{\infty}^{\prime}
$$

for $\mu_{\infty}^{\prime}$-almost every $x \in \Theta_{Q(1)}$. 
Next let $k$ be a large positive integer and set $\Gamma_{k}=\left\{Q \in G: l(Q) \geq 8^{-k}\right\}$. Clearly $\Gamma_{k}$ is closed with respect to the descent relation. For a fixed $p \in(1, \infty)$, we write $u_{k}$ for the $p$-harmonic function, $u_{\Gamma_{k}}$ defined earlier, and $\Omega_{k}=\Omega_{\Gamma_{k}}$. Let

$$
\lambda_{k}=(p-1) \int_{\partial \Omega_{k}}\left|\nabla u_{k}\right|^{p-1} \log \left|\nabla u_{k}\right| d H^{n-1} .
$$

Now we show how to find the sign of $(n-1)-\mathrm{H}-\operatorname{dim} \mu_{\infty}^{\prime}$.

Lemma 7.5. With the above notation,

$$
\lim _{k \rightarrow \infty} \lambda_{k} / k=\mu_{\infty}^{\prime}\left(\mathbb{R}^{n}\right)\left((n-1)-\mathrm{H}-\operatorname{dim} \mu_{\infty}^{\prime}\right) \log 8
$$

Proof. As in the proof of (7.3) we note, for some $\alpha \in[0,1], c$, depending only on $p, n$, that

$$
\mu_{k}\left(\left\{x \in \partial \Omega_{k}: d(x, \partial Q(1))<\epsilon\right\}\right) \leq c \epsilon^{\alpha} .
$$

Let $\mu_{k}^{\prime}$ denote the restriction of $\mu_{k}$ to $\partial \Omega_{k} \cap(Q(1) \times[-1,1])$. Then from (7.10) and Lemmas 3.2-3.6 we see that

$$
\mu_{k}^{\prime} \rightarrow \mu_{\infty}^{\prime} \quad \text { weakly as } k \rightarrow \infty
$$

Next let $Q_{1}, \ldots, Q_{r}$ be the $n-1$-dimensional cubes in $G$ with $l\left(Q_{i}\right)=8^{-k-1}$. Wolff [W2, (2.13)] shows, for $k$ large enough, say $k \geq k_{0}$, that $Q_{i} \subset \partial \Omega_{k}$ for $1 \leq i \leq r$, and that, for some $c=c(n) \geq 1$,

$$
\left(\partial \Omega_{k} \cup \partial \Omega_{\infty}\right) \cap(Q(1) \times[-1,1]) \subset \bigcup_{i=1}^{r} B\left(a_{Q_{i}}, c 8^{-k}\right),
$$

where $a_{Q_{i}}$ is the center of $Q_{i}$. Thus there exist disjoint Borel $H_{i}, 1 \leq i \leq r$, with $Q_{i} \subset H_{i} \subset B\left(a_{Q_{i}}, c 8^{-k}\right)$ and $\bigcup_{i=1}^{r} H_{i}=\partial \Omega_{k} \cap(Q(1) \times[-1,1])$. Given $\delta>0$ we can use Lemma 7.4, the doubling property of $\mu_{\infty}$ and Egoroff type arguments to deduce that if $k_{0}$ is large enough, then for some $c^{\prime}=c^{\prime}(p, n)$, we have

$$
-\frac{k h}{\sigma}(1+\delta) \log 8-c^{\prime} \leq \log \mu_{\infty}^{\prime}\left(B\left(a_{Q_{i}}, c 8^{-k}\right)\right) \leq-\frac{k h}{\sigma}(1-\delta) \log 8+c^{\prime}
$$

except for an exceptional set, say $\beta$, of $i$ with

$$
\sum_{i \in \beta} \mu_{k}^{\prime}\left(H_{i}\right) \leq \delta
$$

Using Lemmas 7.2 and 3.6(ii) we see that if $1 \leq i \leq r$ then for some $\hat{c}=\hat{c}(p, n)$,

$$
\hat{c}^{-1} \leq \frac{\mu_{k}^{\prime}\left(H_{i}\right)}{\mu_{\infty}^{\prime}\left(B\left(a_{Q}, c 8^{-k}\right)\right)} \leq \hat{c} .
$$

Also as in (5.8) we see, for some $\tau \in(0,1)$ depending only on $p, n$ and $1 \leq i \leq r$, that

$$
\tau \log k \leq-\log \mu_{k}^{\prime}\left(H_{i}\right) \leq \tau^{-1} \log k \quad \text { for } k \geq k_{0} .
$$


Using (7.13), (7.14) when $i \notin \beta$, and (7.15) when $i \in \beta$, it follows that, for some $\tilde{c}, c^{*} \geq 1$,

$$
\begin{array}{r}
-k \mu_{k}^{\prime}\left(\mathbb{R}^{n}\right)[h / \sigma(1+\tilde{c} \delta)-(n-1)(1-\tilde{c} \delta)] \log 8-c^{*} \leq \sum_{i=1}^{r} \mu_{k}^{\prime}\left(H_{i}\right) \log \frac{\mu_{k}^{\prime}\left(H_{i}\right)}{H^{n-1}\left(H_{i}\right)} \\
\leq-k \mu_{k}^{\prime}\left(\mathbb{R}^{n}\right)[h / \sigma(1-\tilde{c} \delta)-(n-1)(1+\tilde{c} \delta) \log 8]+c^{*} .
\end{array}
$$

Now $\Omega_{k}$ is Lipschitz on a scale of $8^{-k}$ for $\theta_{0}, N_{0}^{-1}$ small enough so we can use (3.8) to deduce that

$$
\left.\left|\int_{H_{k}}\right| \nabla u_{k}\right|^{p-1} \log \left|\nabla u_{k}\right| d H^{n-1}-\frac{\mu_{k}^{\prime}\left(H_{k}\right)}{p-1} \log \frac{\mu_{k}^{\prime}\left(H_{k}\right)}{H^{n-1}\left(H_{k}\right)} \mid \leq c \mu_{k}^{\prime}\left(H_{k}\right) .
$$

Also, using (3.8) as in (7.17), (5.8) with $\mu$ replaced by $\mu_{k}$, and (6.14) with $\hat{u}$ replaced by $u_{k}$, it follows that if $\partial^{\prime} \Omega_{k}$ denotes the part of $\partial \Omega_{k}$ outside of $Q(1) \times[-1,1]$, then for some $c=c(p, n) \geq 1$,

$$
\int_{\partial^{\prime} \Omega_{k}}\left|\nabla u_{k}\right|^{p-1} \log \left|\nabla u_{k}\right| d H^{n-1} \leq c .
$$

Using (7.17), (7.18) in (7.16), dividing by $k$ and letting $k \rightarrow \infty$, we conclude, for some $\hat{c}=\hat{c}(p, n)$, that

$$
\begin{array}{r}
\liminf _{k \rightarrow \infty} \mu_{k}^{\prime}\left(\mathbb{R}^{n}\right)((n-1)-h / \sigma-\hat{c} \delta) \log 8 \leq \liminf _{k \rightarrow \infty} \lambda_{k} / k, \\
\limsup _{k \rightarrow \infty} \mu_{k}^{\prime}\left(\mathbb{R}^{n}\right)((n-1)-h / \sigma+\hat{c} \delta) \log 8 \geq \limsup _{k \rightarrow \infty} \lambda_{k} / k .
\end{array}
$$

Hence, letting $\delta \rightarrow 0$, using (7.11) and (7.9) we get Lemma 7.5.

Let $\Omega=\left\{x: x_{n}>\phi\left(x^{\prime}\right)\right\}$ and recall, for fixed $p \in(1, \infty)$, that

$$
I=I(\phi, p)=\int_{\partial \Omega}\left|\nabla u^{\prime}\right|^{p-1} \log \left|\nabla u^{\prime}\right| d H^{n-1},
$$

where $u^{\prime}$ is the $p$-harmonic function in Lemma 6.1, defined relative to $\Omega$. To complete the proof of Theorems $2-4$ we prove

Proposition 7.6. If $\theta_{0}$ in (7.1) is sufficiently small, and if $I=I(\phi, p) \neq 0$, then there exist $\beta \in(0,1), c \geq 1$, a positive integer $k_{1}$, depending only on $p, n$, and $\rho=\rho(p, n, N)$ $\in(0, \infty)$ such that the following is true. If $I<0$, then

$$
\int_{\partial \Omega_{k+1}}\left|\nabla u_{k+1}\right|^{p-1} \log \left|\nabla u_{k+1}\right| d H^{n-1} \leq \int_{\partial \Omega_{k}}\left|\nabla u_{k}\right|^{p-1} \log \left|\nabla u_{k}\right| d H^{n-1}+\rho\left(I+c N^{-\beta}\right)
$$

for $k \geq k_{1}$, while if $I>0$ then the above display is valid with $\leq$ replaced by $\geq$ and $c$ by - c. All constants can be chosen independent of $p$ when $p \in[3 / 2,5 / 2]$. 
The proof of Proposition 7.6 will be given after the proof of Lemma 7.2. We now prove Theorems 2-4. For simplicity, we first prove Theorem 3, then Theorem 2, and finally Theorem 4.

Proof of Theorem 3. First, choose $N_{0}$ so large that $c N^{-\beta} \leq|I| / 2$ for $N \geq N_{0}$. Second, iterating the display in Proposition 7.6 from $k=k_{1}$ to $k=m-1$ we see, if $I<0$, that

$$
\begin{aligned}
\int_{\partial \Omega_{m}}\left|\nabla u_{m}\right|^{p-1} \log \left|\nabla u_{m}\right| d H^{n-1} \leq & \int_{\partial \Omega_{k_{1}}}\left|\nabla u_{k_{1}}\right|^{p-1} \log \left|\nabla u_{k_{1}}\right| d H^{n-1} \\
& +\frac{1}{2}\left(m-k_{1}-2\right) \rho I .
\end{aligned}
$$

If $I>0$ we get this inequality with $\leq$ replaced by $\geq$. Moreover, dividing these inequalities by $m$ and letting $m \rightarrow \infty$ we find, using Lemma 7.5, that

$$
\begin{array}{ll}
\mu_{\infty}^{\prime}\left(\mathbb{R}^{n}\right)\left((n-1)-\mathrm{H}-\operatorname{dim} \mu_{\infty}^{\prime}\right) \log 8 \leq \frac{1}{2}(p-1) \rho I & \text { if } I<0, \\
\mu_{\infty}^{\prime}\left(\mathbb{R}^{n}\right)\left((n-1)-\mathrm{H}-\operatorname{dim} \mu_{\infty}^{\prime}\right) \log 8 \geq \frac{1}{2}(p-1) \rho I & \text { if } I>0 .
\end{array}
$$

In particular, if $I<0$, then H-dim $\mu_{\infty}^{\prime}>n-1$, while if $I>0$, then H-dim $\mu_{\infty}^{\prime}<n-1$, and this gives Theorem 3 .

Proof of Theorem 2. We first prove the statement in Theorem 2 made for $p$ fixed, $1<$ $p<n, p \neq 2$. In this case we use Lemma 6.3 to deduce that if $\hat{\phi}=\epsilon \hat{\theta}$, then $\|\nabla \hat{\phi}\|_{\infty} \leq \theta_{0}$ for sufficiently small $\epsilon=\epsilon(p, n)>0$ and $I(\hat{\phi}, p)>0$ for $p>2$ while the reverse inequality holds when $1<p<2$. Using Propositions 6.4 and 6.5, we find that if $1<$ $p<2$, then there exists a piecewise linear $\phi$ with support contained in $\left\{x^{\prime}:\left|x^{\prime}\right|<1 / 2\right\}$ and with $I(\phi, p)<0$, while if $p>2$ then there exists such a $\phi$ with $I(\phi, p)>0$. Using these facts, we can now argue as in the proof of Theorem 3, using Proposition 7.6, and once again choosing $N_{0}$ large enough, to deduce the validity of Theorem 2 for $1<p<n$, $p \neq 2$. If $p \geq n$ in Theorem 2 we can use the fact that $v=\log \left|\nabla u^{\prime}\right|$ is a subsolution to the PDE in (5.1), (5.2) (defined relative to $u^{\prime}$ ) in order to conclude that $I(\phi, p)>0$ for any Lipschitz $\phi \not \equiv 0$ with support in $Q(1)$. In fact, if $L$ is defined relative to $u^{\prime}$ as in (5.1), (5.2), then for $p>n$ we get from (5.7)

$$
L v \geq c^{-1}\left|\nabla u^{\prime}\right|^{p-4} \sum_{i, j=1}^{n}\left(u_{x_{i} x_{j}}^{\prime}\right)^{2}=J
$$

in $\Omega$, where $c=c(p) \rightarrow \infty$ as $p \rightarrow n$, while for $p=n, L v \geq 0$. Now integrating by parts one deduces at least heuristically for $p>n$ that

$$
\int_{\Omega} u^{\prime} J d x \leq \int_{\Omega}\left(u^{\prime} L v-v L u^{\prime}\right) d x=(p-1) I(\phi, p) .
$$

(7.21) can be proved rigorously by first applying the above argument in $\left\{x: u^{\prime}(x)>t\right\}$ $\cap B(0, R)(R>0$ large, $t>0$ small). Letting $R \rightarrow \infty$ and arguing as in (6.21), (6.14), we obtain (7.21) with $\Omega$ replaced by $\Omega(t)=\left\{x \in \Omega: u^{\prime}(x)>t\right\}$. Now from (6.11) for $u^{\prime}$ we see that $\partial \Omega(t)=\left\{x: x_{n}=\psi_{t}\left(x^{\prime}\right)\right\}$ for some smooth $\psi_{t}$ and $\left\|\nabla \psi_{t}\right\|_{\infty} \leq c$, where 
$c$ is independent of $t$ when $0<t<1$. Using this fact, Theorem 3.10(i), and (6.14), we deduce that one can let $t \rightarrow 0$ to get (7.21).

Thus $I(\phi, p)>0$ when $p>n$ unless all second derivatives of $u^{\prime}$ vanish on $\Omega$, which implies that $u^{\prime}(x)=x_{n}$, so $\phi \equiv 0$. If $p=n$ the above argument gives $I(\phi, n)>0$ unless $L v \equiv 0$ in $\Omega$. To show this possibility cannot occur, we note $\nabla u^{\prime} \neq 0$ so by Lemma 3.5, $u^{\prime}$ is real analytic in a neighborhood of each point in $\Omega$. Using this fact and Schwarz reflection we see that if $\hat{x}=\left(x^{\prime}, 0\right),\left|x^{\prime}\right| \geq n$, then $u^{\prime}$ extends to a real analytic $p$-harmonic function in $B(\hat{x}, 1)$ (also denoted $u^{\prime}$ ) with $u^{\prime} \equiv 0$ on $B(\hat{x}, 1) \cap\left\{x: x_{n}=0\right\}$. Then from (5.6) we see that $L v(\hat{x})=0$ only if $u_{x_{i} x_{j}}^{\prime}(\hat{x})=0$ for $1 \leq i, j \leq n$. Thus $\nabla u^{\prime}$ is constant on $\left\{x: x_{n}=0\right\} \backslash B(0, n)$. Using the Cauchy-Kovalevskaya theorem it follows that $u^{\prime}$ is linear in $B(\hat{x}, 1)$. From Lemma 6.1 we find first that $u^{\prime}(x)=x_{n}$ in an open set containing $\partial \Omega \backslash B(0, n)$ and thereupon from real analyticity of $u^{\prime}$ that $u^{\prime}(x) \equiv x_{n}$ and $\phi \equiv 0$. We have reached a contradiction to our assumption that $\phi \not \equiv 0$. Thus $I(\phi, n)>0$ when $\phi \not \equiv 0$ and Theorem 2 is true when $p \geq n$.

Proof of Theorem 4. To prove Theorem 4 note that if $\theta_{0}$ is small enough (independent of $p$ when $p \in[3 / 2,5 / 2])$ and if we have, for some $c=c(n) \geq 1$ and $\eta \in(0,1 / 2)$,

$$
c^{-1} \leq I(\phi, p) / I(\phi, 2) \leq c \quad \text { for } p \in(2-\eta, 2+\eta),
$$

then we can choose $N_{0}$ independent of $p$ when $p \in(2-\eta, 2+\eta)$, so that $c N^{-\beta} \leq$ $|I(\phi, p)| / 2$ whenever $N \geq N_{0}$ and $p \in[2-\eta, 2+\eta]$. From Proposition 7.6 and the above discussion, we see that either $\mathrm{H}-\operatorname{dim} \mu_{\infty}^{\prime}(\cdot, p)>n-1$ or $\mathrm{H}-\operatorname{dim} \mu_{\infty}^{\prime}(\cdot, p)<n-1$ when $p \in[2-\eta, 2+\eta]$. Thus to prove Theorem 4 it suffices to show that (7.22) holds for some $\phi, \eta, c$. To show existence we note that in [W2] examples are provided, when $n=3, p=2$, of smooth $\hat{\phi} \not \equiv 0$ with support in $\left\{x^{\prime}:\left|x^{\prime}\right|<1 / 2\right\}$ for which $I(\hat{\phi}, 2)$ is either positive or negative. Moreover $\|\nabla \hat{\phi}\|_{\infty}$ can be arbitrarily small. These examples generalize easily to $\mathbb{R}^{n}$. More examples of such $\hat{\phi}$ and a rather involved study of $I(\hat{\phi}, 2)$ for various $\hat{\phi}$ can be found in [LVV]. From the above discussion and Propositions 6.4, 6.5, we see there exist a piecewise linear $\phi$ and $\eta>0$ for which (7.1) holds and (7.22) for some $c, \eta$. Furthermore, $I(\phi, \cdot)$ can be either positive or negative on $[2-\eta, 2+\eta]$. Hence we can conclude from Proposition 7.6, as in the proof of Theorem 3, that Theorem 4 is valid.

\subsection{Proof of Lemma 7.2}

In order to complete the proof of Theorems 2-4 it suffices to prove Lemma 7.2 and Proposition 7.6. In this subsection we prove Lemma 7.2. For later use we allow the possibility that $\Gamma^{\prime}=\emptyset$, in which case $\Omega_{\Gamma^{\prime}}=\Omega_{0}=\left\{\left(x^{\prime}, x_{n}\right): x^{\prime} \in \mathbb{R}^{n-1}, x_{n}>0\right\}$. Let $\Gamma^{\prime}, \Gamma, u_{\Gamma}, u_{\Gamma^{\prime}}, \mu_{\Gamma}, \mu_{\Gamma^{\prime}}, r, s$ be as in Lemma 7.2. We assume, as we may, that $\Gamma, \Gamma^{\prime}$ are finite since otherwise we prove the conclusion of Lemma 7.2 for $\Gamma \cap \Gamma_{m}, \Gamma^{\prime} \cap \Gamma_{m}$. Letting $m \rightarrow \infty$, and using Lemmas 3.2-3.6, we then get Lemma 7.2.

For short we write $\Omega^{\prime}, \Omega, u^{\prime}, u, \mu^{\prime}, \mu$ for $\Omega_{\Gamma^{\prime}}, \Omega_{\Gamma}, u_{\Gamma^{\prime}}, u_{\Gamma}, \mu_{\Gamma^{\prime}}, \mu_{\Gamma}$, respectively. From the descent relation we see there exist positive integers $m_{1}<\cdots<m_{k}$ and corresponding domains $\Omega_{i}^{\prime}, 1 \leq i \leq k$, satisfying $\Omega^{\prime}=\Omega_{1}^{\prime}, \Omega=\Omega_{k}^{\prime}$, and for $1 \leq i \leq k, \Omega_{i+1}^{\prime}$ 
is obtained from $\Omega_{i}^{\prime}$ by adding blips to certain $Q \subset \partial \Omega_{i}^{\prime}$ with $l(Q)=8^{-m_{i}}$. Let $u_{i}^{\prime}, \mu_{i}^{\prime}$ be the $p$-harmonic function and associated measure corresponding to $\Omega_{i}^{\prime}$ as in Lemma 6.1 and put $r_{i}=h\left(\Omega_{i}^{\prime}, \Omega_{i+1}^{\prime}\right)$ for $i=1, \ldots, k$. From the Wolff construction we observe for $\theta_{0}, N_{0}^{-1}$ small enough that

$$
r / 2 \leq r_{1} \leq r \quad \text { and } \quad 2 r_{i} \leq r_{i-1}, \quad 2 \leq i \leq k
$$

We prove, under the scenario of Lemma 7.2(a), that

$$
\left|\log \frac{u_{i+1}(x)}{u_{i}(x)}\right| \leq\left(\frac{c r_{i}}{s}\right)^{\alpha}
$$

whenever $x \in \Omega^{\prime}$ with $d\left(x, \partial \Omega^{\prime}\right) \geq s$. Also under the assumptions in Lemma 7.2(b) we will prove that

$$
\left|\log \frac{u_{i+1}(x)}{u_{i}(x)}\right| \leq\left(\frac{c r_{i}}{s}\right)^{\alpha} \frac{\mu^{\prime}\left(B\left(a_{\hat{Q}}, l(Q) / N\right)\right)}{\mu^{\prime}\left(B\left(a_{\hat{Q}}, s\right)\right)}
$$

when $x \in \Omega^{\prime}$ with $\left|x-a_{\hat{Q}}\right| \geq s \geq 100 l(\hat{Q}) / N$. Summing (7.24), (7.25), and using properties of logarithms, as well as (7.23), we obtain Lemma 7.2.

To prove (7.24), (7.25) we need some more notation. Fix $i, 1 \leq i \leq k-1$, and let $K_{i}$ be the set of $Q \in G$ of sidelength $8^{-m_{i}}, Q \subset \partial \Omega_{i}^{\prime}$, that blips are added to in order to get $\Omega_{i+1}^{\prime}$ for $1 \leq i \leq k-1$. Given $Q \in K_{i}$ let $\pi_{Q}(x)$ denote the projection of $\mathbb{R}^{n}$ on the plane containing $Q$. Let $v_{Q}$ denote the inner unit normal to $\Omega_{i}^{\prime}$ at points in $Q$. Then from Section 2 and the discussion at the beginning of Section 7 we see that

$$
\begin{aligned}
\Omega_{i+1}^{\prime} \cap\left(P_{Q} \cup \tilde{P}_{Q}\right) & =\left\{x:\left\langle x-\pi_{Q}(x), v_{Q}\right\rangle>\psi_{Q}\left(\pi_{Q}(x)\right)\right\} \cap\left(P_{Q} \cup \tilde{P}_{Q}\right), \\
\partial \Omega_{i+1}^{\prime} \cap\left(P_{Q} \cup \tilde{P}_{Q}\right) & =\left\{x:\left\langle x-\pi_{Q}(x), v_{Q}\right\rangle=\psi_{Q}\left(\pi_{Q}(x)\right)\right\} \cap\left(P_{Q} \cup \tilde{P}_{Q}\right),
\end{aligned}
$$

where $\psi_{Q}$ is a piecewise linear function defined on the plane through $Q$ with support in $B\left(a_{Q}, l(Q) / 2 N\right)$ Also,

$$
\left\|\nabla \psi_{Q}\right\|_{\infty} \leq \theta_{0} \quad \text { and } \quad r_{i} \leq\left\|\psi_{Q}\right\|_{\infty} \leq 2 r_{i}<l(Q) / N
$$

Finally $\Omega_{i+1}^{\prime}=\Omega_{i}^{\prime}$ outside of $\bigcup_{Q \in K_{i}}\left(P_{Q} \cup \tilde{P}_{Q}\right)$. Choose $\tilde{\psi}_{Q}: Q \rightarrow \mathbb{R}$ to be $C^{\infty}$ with support in $Q \cap B\left(a_{Q}, l(Q) / N\right),\left\|\nabla \tilde{\psi}_{Q}\right\|_{\infty} \leq \theta_{0}$, and $\left\|\tilde{\psi}_{Q}-\psi_{Q}\right\|_{\infty}<10^{-3}\left\|\psi_{Q}\right\|_{\infty}$. Define a deformation $\Omega(t), t \in[0,1]$, of $\Omega_{i}^{\prime}$ as follows: given $Q \in K_{i}$,

$$
\begin{aligned}
\Omega(t) \cap\left(P_{Q} \cup \tilde{P}_{Q}\right) & =\left\{x:\left\langle x-\pi_{Q}(x), v_{Q}\right\rangle>t \tilde{\psi}_{Q}\left(\pi_{Q}(x)\right)\right\} \cap\left(P_{Q} \cup \tilde{P}_{Q}\right), \\
\partial \Omega(t) \cap\left(P_{Q} \cup \tilde{P}_{Q}\right) & =\left\{x:\left\langle x-\pi_{Q}(x), v_{Q}\right\rangle=t \tilde{\psi}_{Q}\left(\pi_{Q}(x)\right)\right\} \cap\left(P_{Q} \cup \tilde{P}_{Q}\right) .
\end{aligned}
$$

Let $\Omega(t)=\Omega_{i}^{\prime}$ outside of $\bigcup_{Q \in K_{i}}\left(P_{Q} \cup \tilde{P}_{Q}\right)$. Note $\Omega(0)=\Omega_{i}^{\prime}$ and $h\left(\Omega(1), \Omega_{i+1}^{\prime}\right) \rightarrow 0$ as

$$
\sum_{Q \in K_{i}}\left\|\psi_{Q}-\tilde{\psi}_{Q}\right\|_{\infty} \rightarrow 0
$$


As in Lemma 7.1 we see that $\Omega(t)$ is Reifenberg flat with constant $\leq c \theta_{0}$, and $\left(P_{Q} \cup \tilde{P}_{Q}\right)$ $\cap \Omega(t)$ is Lipschitz for $t \in[0,1]$. Next for fixed $p \in(1, \infty)$, let $u(\cdot, t), t \in[0,1]$, be the $p$-harmonic function in Lemma 6.1 defined relative to $\Omega(t)$.

Let

$$
U\left(x, t_{1}, t_{2}\right)=\frac{u\left(x, t_{2}\right)-u\left(x, t_{1}\right)}{t_{2}-t_{1}} \quad \text { when } x \in \Omega\left(t_{1}\right) \cap \Omega\left(t_{2}\right) .
$$

We repeat the argument in Section 6 for difference quotients of $u(\cdot, \epsilon)$, only now we use the results from Section 3.2 for Reifenberg flat domains, especially Theorem 3.7. Thus for fixed $t_{1} \neq t_{2} \in[0,1], U\left(\cdot, t_{1}, t_{2}\right)$ satisfies an elliptic equation (see (6.5)) whose ellipticity can be expressed in terms of an $A_{2}$-weight. Hence the results in Section 4 can be used with constants independent of $t_{1}, t_{2}$. Also $U\left(\cdot, t_{1}, t_{2}\right)=0$ on $\partial\left(\Omega\left(t_{2}\right) \cap \Omega\left(t_{1}\right)\right)$ and this function is smooth at points where $U\left(\cdot, t_{1}, t_{2}\right) \neq 0$. Using these facts and arguing as in Section 6 we see first that $U\left(\cdot, t_{1}, t_{2}\right)$ is smooth in the closure of $B\left(a_{Q}, l(Q) / N\right) \cap \Omega\left(t_{2}\right) \cap \Omega\left(t_{1}\right)$ with constants independent of $t_{1}, t_{2}$. Second, from the boundary maximum principle it follows that $U\left(\cdot, t_{1}, t_{2}\right)$ is uniformly bounded in $\Omega\left(t_{2}\right) \cap \Omega\left(t_{1}\right)$. Moreover, from Lemmas 4.1 and 4.2, we see that this function is Hölder continuous and in a certain weighted Sobolev space on $\Omega\left(t_{2}\right) \cap \Omega\left(t_{1}\right)$. The constants are independent of $t_{1}, t_{2} \in[0,1]$. We conclude, as in Section 5, that $u_{t}=\lim _{t_{2} \rightarrow t} U\left(x, t, t_{2}\right)$ exists whenever $t \in[0,1]$ and $x \in \Omega(t)$. Moreover, $u_{t}(\cdot, \cdot), u(\cdot, \cdot)$ are $C^{\infty}$ in the interior of $\bigcup_{t \in[0,1]} \Omega(t) \times\{t\}$ and $C^{\infty}$ in $x, t$ in $\bigcup_{t \in[0,1]} \bar{B}\left(a_{Q}, l(Q) / N\right) \cap \bar{\Omega}(t) \times\{t\}$ whenever $Q \in K_{i}$. Arguing as in (6.19)-(6.20) we find that both $u(\cdot, t)$ and $u_{t}(\cdot, t), t \in[0,1]$, are solutions in $\Omega(t)$ to

$$
L \zeta=\sum_{i, j=1}^{n} \frac{\partial}{\partial x_{i}}\left[b_{i j}(x, t) \zeta_{x_{j}}(x)\right]=0,
$$

where

$$
b_{i j}(x, t)=|\nabla u(x, t)|^{p-4}\left[(p-2) u_{x_{i}}(x, t) u_{x_{j}}(x, t)+\delta_{i j}|\nabla u(x, t)|^{2}\right]
$$

for $1 \leq i, j \leq n$. Furthermore, since $u\left(\pi(x)+t \nu \tilde{\psi}_{Q}\left(\pi_{Q}(x)\right), t\right)=0$ whenever $x \in$ $Q \in K_{i}$, it follows, as in (6.18), from the chain rule and smoothness of $u(\cdot, t), u_{t}(\cdot, t)$ that

$$
u_{t}\left(\pi(x)+t \nu \tilde{\psi}_{Q}\left(\pi_{Q}(x)\right), t\right)=-u_{\nu}\left(\pi_{Q}(x)+t v \tilde{\psi}_{Q}\left(\pi_{Q}(x)\right), t\right) \tilde{\psi}_{Q}\left(\pi_{Q}(x)\right)
$$

whenever $x \in Q \in K_{i}$.

For fixed $t \in[0,1]$, let $g_{t}(\cdot, \cdot)$ be the Green function for the PDE in (7.29), (7.30). We note that Lemmas 4.3-4.4 hold for $g$. Also since $u(\cdot, t)$ is smooth up to the boundary in each $\Omega(t) \cap B\left(a_{Q}, l(Q) / N\right), Q \in K_{i}$, it follows from (4.6) and Schauder type estimates that

$$
u_{t}(x)=-(p-1) \sum_{Q \in K_{i}} \int_{\partial \Omega(t)} u_{v}|\nabla u(\cdot, t)|^{p-2}\left|\nabla g_{t}(x, \cdot)\right| \tilde{\psi}_{Q} d H^{n-1}
$$

whenever $d\left(x, \partial \Omega^{\prime}\right) \geq s \geq 100 r$. 
Our plan is to estimate $\left|u_{t}(x, t)\right|$ in terms of $u(x, t)$ for $t \in[0,1]$, which will then give us an estimate for

$$
\left|\log \frac{u(x, 1)}{u(x, 0)}\right| \leq \int_{0}^{1} \frac{\left|u_{t}(x, t)\right|}{u(x, t)} d t
$$

To do this let $w \in \partial \Omega(t)$ with $|x-w|=\gamma$ and let $a_{\gamma / 2}(w)$ be as in Definition 3.1. Observe from (7.32), (7.27), and Harnack's inequality for positive solutions that

$$
\begin{gathered}
\left|u_{t}(x, t)\right| \leq c r_{i} \sum_{Q \in K_{i}} \int_{\partial \Omega(t) \cap B\left(a_{Q}, l(Q) / N\right)}|\nabla u(\cdot, t)|^{p-1}\left|\nabla g_{t}\left(a_{\gamma / 2}(w), \cdot\right)\right| d H^{n-1} \\
=c r_{i} \sum_{k=0}^{\infty} \sum_{Q \in K_{i}} \int_{\partial \Omega(t) \cap A_{k} \cap B\left(a_{Q}, l(Q) / N\right)}|\nabla u(\cdot, t)|^{p-1}\left|\nabla g_{t}\left(a_{\gamma / 2}(w), \cdot\right)\right| d H^{n-1},
\end{gathered}
$$

where

$$
\begin{aligned}
& A_{k}=\left\{y \in \mathbb{R}^{n}: 2^{k} \gamma \leq|y-w|<2^{k+1} \gamma\right\}, \quad k=1,2, \ldots, \\
& A_{0}=\left\{y \in \mathbb{R}^{n}:|y-w|<2 \gamma\right\} .
\end{aligned}
$$

Let $z \in \partial \Omega(t) \cap A_{k} \cap B\left(a_{Q}, l(Q) / N\right)$ for some $k \in\{0,1, \ldots\}$. Then, using Lemma 4.6, applied to $g_{t}\left(a_{\gamma / 2}(w), \cdot\right)$ and $u(\cdot, t)$, and the interior Harnack inequality as well as l'Hôpital's rule, we see that

$$
\left|\nabla g_{t}\left(a_{\gamma / 2}(w), z\right)\right| \leq c|\nabla u(z, t)| \frac{g_{t}\left(a_{\gamma / 2}(w), a_{2^{k} \gamma}(w)\right)}{u\left(a_{2^{k} \gamma}(w), t\right)}
$$

$H^{n-1}$-almost everywhere on $\partial \Omega(t) \cap A_{k} \cap B\left(a_{Q}, l(Q) / N\right)$. From (7.34), (7.35), we find

$\left|u_{t}(x, t)\right|$

$$
\leq c r_{i} \sum_{k=0}^{\infty} \frac{g_{t}\left(a_{\gamma / 2}(w), a_{2^{k} \gamma}(w)\right)}{u\left(a_{2^{k} \gamma}(w), t\right)} \sum_{Q \in K_{i}} \int_{\partial \Omega(t) \cap A_{k} \cap B\left(a_{Q}, l(Q) / N\right)}|\nabla u(\cdot, t)|^{p} d H^{n-1} .
$$

To estimate the integrals in (7.36) we note that $\partial \Omega(t) \cap B\left(a_{Q}, 4 l(Q) / N\right), Q \in K_{i}$, is Lipschitz on a scale $r_{i}$ and each point of this set lies within $r_{i}$ of a point where $u(\cdot, t)$ is zero. Second, we apply Theorem 3.10(ii), (iv) to $u(\cdot, t)$, and then use Lemma 3.6(ii) to deduce that if $\zeta \in \partial \Omega(t) \cap A_{k} \cap B\left(a_{Q}, l(Q) / N\right)$, then

$$
\int_{\partial \Omega(t) \cap B\left(\zeta, r_{i}\right) \cap A_{k}}|\nabla u(\cdot, t)|^{p} d H^{n-1} \leq c r_{i}^{-1} u\left(a_{r_{i}}(\zeta), t\right) \mu_{t}\left(B\left(\zeta, r_{i}\right) \cap A_{k}\right),
$$

where $\mu_{t}$ is the measure associated with $u(\cdot, t)$. Also using Lemma 3.3 and the Harnack inequality for $p$-harmonic functions, we find that

$$
u\left(a_{r_{i}}(\zeta), t\right) \leq c\left(r_{i} / \gamma\right)^{\alpha} 2^{-k \alpha} u\left(a_{2^{k} \gamma}(w), t\right)
$$


Combining (7.37), (7.38), using a covering lemma, and the doubling property of $\mu_{t}$, we see that

$$
\begin{aligned}
\sum_{Q \in K_{i}} \int_{\partial \Omega(t) \cap A_{k} \cap B\left(a_{Q}, l(Q) / N\right)}|\nabla u(\cdot, t)|^{p} d H^{n-1} \\
\leq c r_{i}^{-1}\left(r_{i} / \gamma\right)^{\alpha} 2^{-k \alpha} u\left(a_{2^{k} \gamma}(w), t\right) \mu_{t}\left(\partial \Omega(t) \cap A_{k}\right) .
\end{aligned}
$$

Using (7.39) in (7.36) we find, after dividing by $u\left(a_{\gamma}(w), t\right)$ and using the Harnack inequality, that

$$
\frac{\left|u_{t}(x, t)\right|}{u(x, t)} \leq c\left(r_{i} / \gamma\right)^{\alpha} \sum_{k=0}^{\infty} 2^{-k \alpha} \frac{g_{t}\left(a_{\gamma / 2}(w), a_{2^{k} \gamma}(w)\right)}{u\left(a_{\gamma}(w), t\right)} \mu_{t}\left(\partial \Omega(t) \cap A_{k}\right) .
$$

Applying Lemma 4.6 to the functions $g_{t}\left(\cdot, a_{2^{k} \gamma}(w)\right), u(\cdot, t)$, using also the Harnack inequality, we can continue the estimate in (7.40) to conclude that

$$
\frac{\left|u_{t}(x, t)\right|}{u(x, t)} \leq c\left(r_{i} / \gamma\right)^{\alpha} \sum_{k=0}^{\infty} 2^{-k \alpha} \frac{g_{t}\left(a_{2^{k-1} \gamma}(w), a_{2^{k} \gamma}(w)\right)}{u\left(a_{2^{k} \gamma(w)}, t\right)} \mu_{t}\left(\partial \Omega(t) \cap A_{k}\right) .
$$

From Lemmas 4.3(a), 3.6(ii), and Theorem 3.7, we get

$$
g_{t}\left(a_{2^{k-1} \gamma}(w), a_{2^{k} \gamma}(w)\right) \leq c\left(2^{k} \gamma\right)^{2-n}\left|\nabla u\left(a_{2^{k} \gamma}(w), t\right)\right|^{2-p} \leq c \frac{u\left(a_{2^{k} \gamma}(w), t\right)}{\mu_{t}\left(\partial \Omega(t) \cap A_{k}\right)} .
$$

Combining (7.42), (7.41) we find that

$$
\frac{\left|u_{t}(x, t)\right|}{u(x, t)} \leq c\left(r_{i} / \gamma\right)^{\alpha} \sum_{k=0}^{\infty} 2^{-k \alpha} \leq c^{2}\left(r_{i} / \gamma\right)^{\alpha}
$$

where $c$ depends only on $p, n$ but can be chosen independent of $p$ when $p \in[3 / 2,5 / 2]$. Using (7.43) in (7.33) we obtain

$$
\left|\log \frac{u(x, 1)}{u(x, 0)}\right| \leq c\left(r_{i} / \gamma\right)^{\alpha}
$$

Letting $\|\tilde{\psi}-\psi\|_{\infty} \rightarrow 0$ we conclude from this inequality, from $\gamma \geq s / 2$, and from our earlier remarks that first (7.24) is valid and second that (a) in Lemma 7.2 is true.

To prove the validity of (b) in Lemma 7.2 we use the same notation as in the proof of (a). Let $\rho=4 l(\hat{Q}) / N, \tau=\left|x-a_{\hat{Q}}\right|$, and let $\zeta$ be a point in $\partial \Omega(t)$ with $\left|\zeta-a_{\hat{Q}}\right|<\rho$. Note that $u_{t}=0$ on $\partial \Omega(t) \backslash B\left(a_{\hat{Q}}, \rho\right)$. Using this and arguing as in (7.32)-(7.36) we get

$$
\begin{aligned}
\left|u_{t}(x, t)\right| & \leq c r_{i} \int_{\partial \Omega(t) \cap B\left(a_{\hat{Q}}, \rho\right)}|\nabla u(\cdot, t)|^{p-1}\left|\nabla g_{t}(x, \cdot)\right| d H^{n-1} \\
& \leq c r_{i} \frac{g_{t}\left(x, a_{\tau}(\zeta)\right)}{u\left(a_{\tau}(\zeta)\right)} \int_{\partial \Omega(t) \cap B\left(a_{\hat{Q}}, \rho\right)}|\nabla u(\cdot, t)|^{p} d H^{n-1} .
\end{aligned}
$$


Now arguing as in (7.37)-(7.39) we obtain

$$
\int_{\partial \Omega(t) \cap B\left(a_{\hat{Q}}, \rho\right)}|\nabla u(\cdot, t)|^{p} d H^{n-1} \leq c r_{i}^{-1}\left(r_{i} / \tau\right)^{\alpha} u\left(a_{\tau}(\zeta)\right) \mu_{t}\left(B\left(a_{\hat{Q}}, \rho\right)\right) .
$$

Moreover the analogue of (7.42) is

$$
g_{t}\left(x, a_{\tau}(\zeta)\right) \leq c \frac{u(x, t)}{\mu_{t}\left(B\left(a_{\hat{Q}}, \tau\right)\right)}
$$

Using the above inequalities in (7.44) we get

$$
\frac{\left|u_{t}(x, t)\right|}{u(x, t)} \leq c\left(r_{i} / \tau\right)^{\alpha} \frac{\mu_{t}\left(B\left(a_{\hat{Q}}, \rho\right)\right)}{\mu_{t}\left(B\left(a_{\hat{Q}}, \tau\right)\right)} .
$$

We claim that

$$
\frac{\mu_{t}\left(B\left(a_{\hat{Q}}, \rho\right)\right)}{\mu_{t}\left(B\left(a_{\hat{Q}}, \tau\right)\right)} \approx \frac{\mu^{\prime}\left(B\left(a_{\hat{Q}}, \rho\right)\right)}{\mu^{\prime}\left(B\left(a_{\hat{Q}}, \tau\right)\right)},
$$

where $\approx$ means the two ratios are bounded above and below by constants depending only on $p, n$, and these constants can be chosen independent of $p$ when $p \in[3 / 2,5 / 2]$. Indeed from (7.23), (7.24), (7.43), and integration we find that

$$
u\left(a_{\xi}(\zeta), t\right) \approx u^{\prime}\left(a_{\xi}(\zeta)\right) \quad \text { whenever } \xi=\rho \text { or } \tau \text { and } t \in[0,1] .
$$

This fact, Lemma 3.6(ii), and Harnack's inequality (Lemma 3.2(ii)) imply (7.46). Using (7.46) in (7.45), integrating, and using the doubling property of $\mu^{\prime}$, we get

$$
\left|\log \frac{u(x, 1)}{u(x, 0)}\right| \leq c\left(r_{i} / \tau\right)^{\alpha} \frac{\mu^{\prime}\left(B\left(a_{\hat{Q}}, l(\hat{Q}) / N\right)\right)}{\mu^{\prime}\left(B\left(a_{\hat{Q}}, \tau\right)\right)} .
$$

Letting $\|\tilde{\psi}-\psi\|_{\infty} \rightarrow 0$ we conclude from the above inequality, from $\tau \geq s$, and from our earlier remarks that Lemma 7.2(b) is valid. The proof of Lemma 7.2 is now complete.

\subsection{Preliminary reductions for the proof of Proposition 7.6}

In this section we use the game plan in [W2] to estimate the integrals in Proposition 7.6. To begin, we prove

Lemma 7.7. Let $\Gamma^{\prime} \subset \Gamma \subset G$ and suppose that $\Gamma, \Gamma^{\prime}$ are closed under the descent relation. For fixed $p \in(1, \infty)$, let $u_{\Gamma}, u_{\Gamma^{\prime}}, \mu_{\Gamma}, \mu_{\Gamma^{\prime}}$ be the corresponding $p$-harmonic functions and measures, defined relative to $\Omega_{\Gamma}, \Omega_{\Gamma^{\prime}}$ as in Lemma 6.1. Given $\eta>0$ there exists $R=R(\eta)>0$ such that

$$
\left|\mu_{\Gamma}(S)-\mu_{\Gamma^{\prime}}(S)\right| \leq \eta \quad \text { whenever } S \text { is a Borel set with } B(0, R) \subset S .
$$


Proof. To simplify we once again let $u=u_{\Gamma}, \mu=\mu_{\Gamma}, u^{\prime}=u_{\Gamma^{\prime}}, \mu^{\prime}=\mu_{\Gamma^{\prime}}$. Recall from Section 2 that $\Omega_{0}=\left\{\left(x^{\prime}, x_{n}\right): x^{\prime} \in \mathbb{R}^{n-1}, x_{n}>0\right\}$. For $\theta_{0}, N_{0}^{-1}$ small enough we see from Lemma 7.1 that Theorem 3.7 can be used in place of (6.11) to get (see (6.14))

$$
\left|e_{n}-\nabla \hat{u}(x)\right|+\left|e_{n}-\nabla u^{\prime}(x)\right| \leq \tilde{c}|x|^{1-n-\sigma}
$$

for some $\sigma>0$, depending on $p, n$, whenever $x \in \bar{\Omega}_{0} \backslash B(0, n)$. Also from Theorem 3.10 we have

$$
d \mu=|\nabla u|^{p-1} d H^{n-1} \quad \text { and } \quad d \mu^{\prime}=\left|\nabla u^{\prime}\right|^{p-1} d H^{n-1} \quad \text { on } \partial \Omega_{0} \backslash B(0, n) .
$$

Using (7.47) and (7.48) we deduce, for $R>0$ large enough, that

$$
\begin{aligned}
\mid \mu(S \backslash B(0, R))-\mu^{\prime} & (S \backslash B(0, R)) \mid \\
\leq c & \left.\int_{\partial \Omega_{0} \backslash B(0, R)}|| \nabla u\right|^{p-1}-\left|\nabla u^{\prime}\right|^{p-1} \mid d H^{n-1} \leq \eta / 2 .
\end{aligned}
$$

Next suppose that $0 \leq \theta \in C_{0}^{\infty}(B(0,2 R))$ with $\theta \equiv 1$ on $B(0, R)$ and $|\nabla \theta| \leq c / R$. Using $\theta$ as a test function in Lemma 3.6(i), for $u$ and for $u^{\prime}$, we see that

$$
\int\left\langle\left(|\nabla u|^{p-2} \nabla u-\left|\nabla u^{\prime}\right|^{p-2} \nabla u^{\prime}\right), \nabla \theta\right\rangle d x=-\int \theta\left(d \mu-d \mu^{\prime}\right) .
$$

The left-hand integral in (7.50) is easily estimated using (7.47) and the fact that $\nabla \theta=0$ in $B(0, R)$. As for the right-hand integral in (7.50) we divide it into integrals over $B(0, R)$ and $\mathbb{R}^{n} \backslash B(0, R)$. The integral over the last set is estimated using the same strategy as in the proof of (7.49). We deduce from (7.50) for $R$ large enough that

$$
\left|\mu(B(0, R))-\mu^{\prime}(B(0, R))\right| \leq \eta / 2 .
$$

Combining this inequality with (7.49) we conclude that Lemma 7.7 is true.

Our purpose in the following is to reduce the proof of Proposition 7.6 to the proof of a quite technically involved lemma which we then prove in the next subsection. Recall, in the context of Proposition 7.6, that $\psi\left(x^{\prime}\right)=\phi\left(N x^{\prime}\right) / N, x^{\prime} \in \mathbb{R}^{n-1}$, and let $\tilde{u}_{1}$ be the $p$-harmonic function in $\left\{\left(x^{\prime}, x_{n}\right): x_{n}>\psi\left(x^{\prime}\right)\right\}$ guaranteed by Lemma 6.1. Let $u_{1}(x)=$ $\tilde{u}_{1}(N x) / N$. Then $u_{1}$ is $p$-harmonic in $\left\{\left(x^{\prime}, x_{n}\right): x_{n}>\phi\left(x^{\prime}\right)\right\}$ and $\left|x_{n}-u_{1}(x)\right| \rightarrow 0$ as $x \rightarrow \infty$. Using this observation, uniqueness in Lemma 6.1, and changing variables we have

$$
I(\psi, p)=I(\phi, p) / N^{n-1} .
$$

Recall from Subsection 7.1 that $\Omega_{m}=\Omega_{\Gamma_{m}}$ where $\Gamma_{m}=\left\{Q \in G: l(Q) \geq 8^{-m}\right\}$, $m=1,2, \ldots$ Also for $k$ a fixed and large positive integer, $Q_{1}, \ldots, Q_{r} \subset \partial \Omega_{k}$ were the $n-1$-dimensional cubes of sidelength $8^{-k-1}$ in $G$ that blips were added to in order to get $\Omega_{k+1}$. Following Wolff we define intermediary domains, $\Omega_{k}^{i}, 1 \leq i \leq r+1$, as follows: $\Omega_{k}^{1}=\Omega_{k}$, while for $1 \leq i \leq r, \Omega_{k}^{i+1}$ is obtained from $\Omega_{k}^{i}$ by adding a blip along $Q_{i}$. Thus $\Omega_{k+1}=\Omega_{k}^{r+1}$. Below we show that Proposition 7.6 is an easy consequence of the following lemma. 
Lemma 7.8. For fixed $p \in(1, \infty)$, let $u_{k}^{j}, \mu_{k}^{j}, 1 \leq j \leq r+1$, be the $p$-harmonic function and measure corresponding to $\Omega_{k}^{j}$ as in Lemma 6.1. Assume $I=I(\phi, p) \neq 0$ Then for $\theta_{0}, N_{0}^{-1}$ sufficiently small there exist $\beta \in(0,1), c \geq 1$, a positive integer $k_{1}$, and $\xi \in(0, \infty)$, depending only on $p, n$, such that if $I<0$, then

$$
\begin{aligned}
\int_{\partial \Omega_{k}^{j+1}}\left|\nabla u_{k}^{j+1}\right|^{p-1} \log \left|\nabla u_{k}^{j+1}\right| & d H^{n-1} \leq \int_{\partial \Omega_{k}^{j}}\left|\nabla u_{k}^{j}\right|^{p-1} \log \left|\nabla u_{k}^{j}\right| d H^{n-1} \\
& +\xi\left(N^{n-1} I(\psi, p)+c N^{-\beta}\right) \mu_{k}^{j}\left(B\left(a_{Q_{j}}, 100 \cdot 8^{-k} / N\right)\right)
\end{aligned}
$$

for $1 \leq j \leq r$, while if $I>0$ then the above display is valid with $\leq$ replaced by $\geq$ and $c$ by $-c$. Moreover, the constants $\xi, \beta, c, k_{1}$ can be chosen independent of $p$ when $p \in[3 / 2,5 / 2]$.

Proof of Proposition 7.6 assuming Lemma 7.8. We only supply the proof when $I<0$. The proof is essentially unchanged if $I>0$. We observe that if

$$
\Gamma_{k}^{j}=\Gamma_{k} \cup\left\{Q_{1}, \ldots, Q_{j}\right\}
$$

then $\Gamma_{k}^{j}$ is closed under the descent relation for $1 \leq j \leq r+1$ and $\Gamma_{k}^{j} \subset \Gamma_{k}^{i}$ whenever $1 \leq j \leq i \leq r+1$. Thus we can apply Lemma 7.2 to get $u_{k}^{i}(x) \approx u_{k}^{j}(x)$ whenever $x \in \Omega_{k}$ with $d\left(x, \partial \Omega_{k}\right) \geq 100 h\left(\Omega_{k}, \Omega_{k+1}\right)$. Using this, and Lemma 3.6(ii), we find that

$$
\mu_{k}^{l}\left(B\left(a_{Q_{j}}, 100 \cdot 8^{-k} / N\right)\right) \approx \mu_{k}^{m}\left(B\left(a_{Q_{j}}, 100 \cdot 8^{-k} / N\right)\right)
$$

whenever $1 \leq j, l, m \leq r+1$. The proportionality constants depend only on $p, n$, and can be chosen independent of $p$ when $p \in[3 / 2,5 / 2]$. To get Proposition 7.6 we iterate the display in Lemma 7.8 starting at $j=1$ and finishing with $j=r$. In this way we get a sum involving various measures. In view of (7.52) we can replace all these measures by $\mu_{k}=\mu_{k}^{1}$. Doing this and using (7.51) we get

$$
\begin{aligned}
\int_{\partial \Omega_{k+1}}\left|\nabla u_{k+1}\right|^{p-1} \log \left|\nabla u_{k}\right| & d H^{n-1} \leq \int_{\partial \Omega_{k}}\left|\nabla u_{k}\right|^{p-1} \log \left|\nabla u_{k}\right| d H^{n-1} \\
& +c \xi\left(I(\phi, p)+c N^{-\beta}\right) \mu_{k}\left(\bigcup_{j=1}^{r} B\left(a_{Q_{j}}, 8^{-k} / N\right)\right) .
\end{aligned}
$$

From the doubling property of $\mu$ and the Wolff construction (see [W2, (2.13)]) it follows that

$$
\hat{c}^{-1} \leq \mu_{k}\left(\bigcup_{j=1}^{r} B\left(a_{Q_{j}}, 8^{-k-1} / N\right)\right) \leq \tilde{c},
$$

where $\hat{c}=\hat{c}(p, n, N), \tilde{c}=\tilde{c}(p, n) \geq 1$. Using this inequality in (7.53) we get Proposition 7.6. 


\subsection{Proof of Lemma 7.8}

To prove Lemma 7.8 fix $k, j, 1 \leq j \leq r$, and $p \in(1, \infty)$. Then $\Omega_{k}^{j+1}$ is obtained from $\Omega_{k}^{j}$ by adding a blip along $Q_{j}$. Let $t=8^{-k-1}$ and let $T$ be the conformal affine mapping introduced in Section 2. Then $T(Q(1))=Q_{j}$ and if $\tilde{\Omega}=T^{-1}\left(\Omega_{k}^{j+1}\right), \Omega=T^{-1}\left(\Omega_{k}^{j}\right)$, then $\tilde{\Omega}$ is obtained from $\Omega$ by adding the blip $\Lambda$ to $Q(1)$ in (2.12). Let $u(x)=t^{-1} u_{k}^{j}(T x)$ and $\tilde{u}(x)=t^{-1} u_{k}^{j+1}(T x), x \in \mathbb{R}^{n}$. Since the $p$-Laplacian is invariant under conformal affine mappings and multiplication by a constant, we see that $u, \tilde{u}$ are $p$-harmonic in $\Omega, \tilde{\Omega}$, respectively, and continuous on $\mathbb{R}^{n}$ with $u \equiv 0$ on $\mathbb{R}^{n} \backslash \Omega$ while $\tilde{u} \equiv 0$ on $\mathbb{R}^{n} \backslash \tilde{\Omega}$. Let $\mu, \tilde{\mu}$ be the measures associated with $u, \tilde{u}$, respectively. Observe that if $F$ is a Borel subset of $\partial \Omega$, then

$$
\begin{aligned}
H^{n-1}(T(F)) & =t^{n-1} H^{n-1}(F), \\
\mu_{k}^{j}(T(F)) & =t^{n-1} \mu(F) .
\end{aligned}
$$

Similar equalities hold when $F \subset \partial \tilde{\Omega}$. From (7.54) and Lemma 7.7 we see that

$$
\begin{aligned}
& \lim _{R \rightarrow \infty}\left(\int_{\partial \tilde{\Omega} \cap B(0, R)}|\nabla \tilde{u}|^{p-1} \log |\nabla \tilde{u}| d H^{n-1}-\int_{\partial \Omega \cap B(0, R)}|\nabla u|^{p-1} \log |\nabla u| d H^{n-1}\right) \\
& =t^{1-n}\left(\int_{\partial \Omega_{k}^{j+1}}\left|\nabla u_{k}^{j+1}\right|^{p-1} \log \left|\nabla u_{k}^{j+1}\right| d H^{n-1}-\int_{\partial \Omega_{k}^{j}}\left|\nabla u_{k}^{j}\right|^{p-1} \log \left|\nabla u_{k}^{j}\right| d H^{n-1}\right) .
\end{aligned}
$$

From this deduction and (7.54) we see that to prove the inequality in Lemma 7.8 it suffices to prove a similar inequality involving $u, \tilde{u}, \Omega, \tilde{\Omega}$, and with $Q(1)$ replacing $Q_{j}$. For this purpose let $\lambda=|\nabla u(0)|$. Then, using Theorem 3.9 applied to the functions $u$ and $x_{n}$, l'Hôpital's rule, Theorem 3.10 and Lemma 3.6, we see that

$$
\lambda=|\nabla u(0)| \approx\left(\mu(B(0, \rho)) / \rho^{n-1}\right)^{1 /(p-1)} \quad \text { whenever } 0<\rho \leq 1 .
$$

From (7.55), as well as (7.54), Lemma 7.2(b) applied to $u_{k}^{j}, u_{k}^{j+1}, \Omega_{k}^{j}, \Omega_{k}^{j+1}$, and l'Hôpital's rule we see that if $u^{*}, u^{* *}$ denote either of $\tilde{u}, u$, then

$$
\left|\frac{\left|\nabla u^{*}(z)\right|}{\left|\nabla u^{* *}(z)\right|}-1\right| \leq c \frac{\theta_{0}^{\alpha} \mu(B(0,1 / N))}{(|z| N)^{\alpha} \mu(B(0,|z|))} \leq c^{2} \frac{\theta_{0}^{\alpha} N^{1-n} \lambda^{p-1}}{(|z| N)^{\alpha} \mu(B(0,|z|))}
$$

whenever $z \in \partial \Omega \backslash B(0,100 / N)$. Also from Lemmas 7.2(a) and 3.6(ii) we have

$$
c^{-1} \mu(B(0, \rho)) \leq \tilde{\mu}(B(0, \rho)) \leq c \mu(B(0, \rho))
$$

for some $c=c(p, n)$ whenever $\rho \geq 1 / N$. For fixed $R \geq 10^{10}$ we intend to estimate

$$
\begin{aligned}
\hat{I}=\hat{I}(R):= & \int_{\partial \tilde{\Omega} \cap B(0, R)} \lambda^{-(p-1)}|\nabla \tilde{u}|^{p-1} \log \left(\lambda^{-1}|\nabla \tilde{u}|\right) d H^{n-1} \\
& -\int_{\partial \Omega \cap B(0, R)} \lambda^{-(p-1)}|\nabla u|^{p-1} \log \left(\lambda^{-1}|\nabla u|\right) d H^{n-1} .
\end{aligned}
$$

Letting $R \rightarrow \infty$, using our estimate, Lemma 7.7, and (7.54), we will then get Lemma 7.8. 
For this purpose, and for a parameter $\gamma \in(0,1)$ to be fixed, we put

$$
\partial \Omega=\left(\partial \Omega \cap B\left(0, N^{-\gamma}\right)\right) \cup \bigcup_{k=1}^{\infty} E_{k},
$$

where

$$
E_{k}=\partial \Omega \cap B(0, R) \cap\left(B\left(0,2^{k} N^{-\gamma}\right) \backslash B\left(0,2^{k-1} N^{-\gamma}\right)\right) .
$$

Using this notation we let

$$
\begin{aligned}
J= & \int_{\partial \tilde{\Omega} \cap B\left(0, N^{-\gamma}\right)} \lambda^{-(p-1)}|\nabla \tilde{u}|^{p-1} \log \left(\lambda^{-1}|\nabla \tilde{u}|\right) d H^{n-1} \\
& -\int_{\partial \Omega \cap B\left(0, N^{-\gamma}\right)} \lambda^{-(p-1)}|\nabla u|^{p-1} \log \left(\lambda^{-1}|\nabla u|\right) d H^{n-1}
\end{aligned}
$$

and

$$
\begin{aligned}
I_{k}= & \int_{E_{k}} \lambda^{-(p-1)}|\nabla \tilde{u}|^{p-1} \log \left(\lambda^{-1}|\nabla \tilde{u}|\right) d H^{n-1} \\
& -\int_{E_{k}} \lambda^{-(p-1)}|\nabla u|^{p-1} \log \left(\lambda^{-1}|\nabla u|\right) d H^{n-1}
\end{aligned}
$$

whenever $k$ is a positive integer. Hence,

$$
\hat{I}=J+\sum_{k=1}^{\infty} I_{k} .
$$

We first focus on the term $I_{k}$ for $k=1,2, \ldots$ For fixed $k$ we write

$$
\begin{aligned}
I_{k}= & \int_{E_{k}} \lambda^{-(p-1)}\left[|\nabla \tilde{u}|^{p-1} \log \left(\lambda^{-1}|\nabla \tilde{u}|\right)-|\nabla \tilde{u}|^{p-1} \log \left(\lambda^{-1}|\nabla u|\right)\right] d H^{n-1} \\
& +\int_{E_{k}} \lambda^{-(p-1)}\left[|\nabla \tilde{u}|^{p-1} \log \left(\lambda^{-1}|\nabla u|\right)-|\nabla u|^{p-1} \log \left(\lambda^{-1}|\nabla u|\right)\right] d H^{n-1} \\
= & \int_{E_{k}} \lambda^{-(p-1)}|\nabla \tilde{u}|^{p-1} \log \frac{|\nabla \tilde{u}|}{|\nabla u|} d H^{n-1} \\
& +\int_{E_{k}} \lambda^{-(p-1)}\left[|\nabla \tilde{u}|^{p-1}-|\nabla u|^{p-1}\right] \log \left(\lambda^{-1}|\nabla u|\right) d H^{n-1} \\
= & : I_{k}^{1}+I_{k}^{2} .
\end{aligned}
$$

Using (7.56), (7.57), and the fact that $|\log x| \approx|1-x|$ on $(1 / 2,2)$ we find, for some $c=c(p, n)$, that

$$
\begin{aligned}
\left|I_{k}^{1}\right| & \leq c \int_{E_{k}} \lambda^{-(p-1)}|\nabla \tilde{u}(z)|^{p-1}\left|\frac{|\nabla \tilde{u}(z)|}{|\nabla u(z)|}-1\right| d H^{n-1} \\
& \leq \frac{c^{2} \theta_{0}^{\alpha} \tilde{\mu}\left(B\left(0,2^{k} N^{-\gamma}\right)\right)}{2^{k \alpha} N^{(1-\gamma) \alpha+n-1} \mu\left(B\left(0,2^{k-1} N^{-\gamma}\right)\right)} \leq \frac{c^{3} \theta_{0}^{\alpha}}{2^{k \alpha} N^{(1-\gamma) \alpha+n-1}} .
\end{aligned}
$$


Hence

$$
\sum_{k=1}^{\infty}\left|I_{k}^{1}\right| \leq c N^{-(1-\gamma) \alpha+1-n}
$$

We next estimate $I_{k}^{2}$. Observe from (7.56) and l'Hôpital's rule that

$$
\begin{aligned}
\left.|| \nabla u(z)\right|^{p-1}-|\nabla \tilde{u}(z)|^{p-1} \mid & \leq c|\nabla u(z)|^{p-1}\left|1-\frac{|\nabla u(z)|}{|\nabla \tilde{u}(z)|}\right| \\
& \leq c^{2}|\nabla u(z)|^{p-1} \frac{\theta_{0}^{\alpha} N^{1-n} \lambda^{p-1}}{(|z| N)^{\alpha} \mu(B(0,|z|))}
\end{aligned}
$$

whenever $z \in E_{k}$. Using (7.65) in the definition of $I_{k}^{2}$ we obtain

$$
\left|I_{k}^{2}\right| \leq \frac{c \theta_{0}^{\alpha} N^{1-n}}{\left(2^{k-1} N^{1-\gamma}\right)^{\alpha} \mu\left(B\left(0,2^{k-1} N^{-\gamma}\right)\right)}\left|T_{k}\right|,
$$

where

$$
T_{k}=\int_{E_{k}}|\nabla u|^{p-1}\left|\log \left(\lambda^{-1}|\nabla u|\right)\right| d H^{n-1} .
$$

Let $A_{k}=\left\{\Delta_{k j}\right\}=\left\{\Delta_{k j}\left(w_{k j}, r_{k j}\right)\right\}=\left\{\partial \Omega \cap B\left(w_{k j}, r_{k j}\right)\right\}$, where $w_{k j} \in \partial \Omega, r_{k j} \approx N^{-\gamma}$, is a covering of $E_{k}$ such that $\Delta_{k j}\left(w_{k j}, r_{k j} / 4\right) \cap \Delta_{k j}\left(w_{k j^{\prime}}, r_{k j^{\prime}} / 4\right)=\emptyset$ whenever $j \neq j^{\prime}$. Then

$$
\begin{aligned}
\left|T_{k}\right| \leq & \sum_{j} \int_{\Delta_{k j}}|\nabla u|^{p-1}\left|\log \left(\lambda^{-1}|\nabla u|\right)\right| d H^{n-1} \\
\leq & \sum_{j} \int_{\Delta_{k j}}|\nabla u|^{p-1}\left|\log \left(N^{\gamma(n-1) /(p-1)} \lambda^{-1}\left(\mu\left(\Delta_{k j}\right)\right)^{1 /(p-1)}\right)\right| d H^{n-1} \\
& +\sum_{j} \int_{\Delta_{k j}}|\nabla u|^{p-1}\left|\log \left(N^{-\gamma(n-1) /(p-1)}|\nabla u|\left(\mu\left(\Delta_{k j}\right)\right)^{-1 /(p-1)}\right)\right| d H^{n-1} \\
= & : \sum_{j}\left(T_{k j}^{1}+T_{k j}^{2}\right) .
\end{aligned}
$$

To estimate $T_{k j}^{1}$ we first note, using (7.55), that

$$
N^{\gamma(n-1) /(p-1)} \lambda^{-1}\left(\mu\left(\Delta_{k j}\right)\right)^{1 /(p-1)} \approx\left(\frac{\mu\left(\Delta_{k j}\right)}{\mu\left(B\left(0, N^{-\gamma}\right)\right)}\right)^{1 /(p-1)} .
$$

Using (7.68) in the definition of $T_{k j}^{1}$ we get

$$
T_{k j}^{1} \leq c\left|\mu\left(\Delta_{k j}\right) \log \frac{\mu\left(\Delta_{k j}\right)}{\mu\left(B\left(0, N^{-\gamma}\right)\right)}\right| \leq c k \mu\left(\Delta_{k j}\right),
$$


where the last inequality follows from the doubling property for the measure $\mu$. To estimate $T_{k j}^{2}$ we use (3.8), which yields

$$
\begin{aligned}
T_{k j}^{2} & \leq c \mu\left(\Delta_{k j}\right)\left|\log \left(c N^{-\gamma(n-1) /(p-1)}\left(\mu\left(\Delta_{k j}\right)\right)^{-1 /(p-1)}\left(\frac{\mu\left(\Delta_{k j}\right)}{H^{n-1}\left(\Delta_{k j}\right)}\right)^{1 /(p-1)}\right)\right| \\
& \leq c \mu\left(\Delta_{k j}\right) .
\end{aligned}
$$

Putting (7.69), (7.70) together we can first conclude, by standard arguments, that

$$
\left|T_{k}\right| \leq \sum_{j}\left(T_{k j}^{1}+T_{k j}^{2}\right) \leq c k \mu\left(E_{k}\right)
$$

and then from (7.66), (7.71) that

$$
\left|I_{k}^{2}\right| \leq \frac{c k \theta_{0}^{\alpha} N^{1-n} \mu\left(E_{k}\right)}{\left(2^{k-1} N^{1-\gamma}\right)^{\alpha} \mu\left(B\left(0,2^{k-1} N^{-\gamma}\right)\right)} \leq \frac{c^{2} k \theta_{0}^{\alpha} N^{1-n}}{\left(2^{k-1} N^{1-\gamma}\right)^{\alpha}} .
$$

Summing over $k$ we see first that

$$
\sum_{k=1}^{\infty}\left|I_{k}^{2}\right| \leq c N^{-(1-\gamma) \alpha+1-n},
$$

and then, from this inequality and (7.64), that

$$
\sum_{k=1}^{\infty}\left|I_{k}\right| \leq c N^{-(1-\gamma) \alpha+1-n}
$$

where $c$ depends only on $p, n$ and can be chosen independent of $p$ when $p \in[3 / 2,5 / 2]$. This completes the estimate of $|\hat{I}-J|$.

Next we focus on estimating $J$ in (7.59). As in (7.51) let $\tilde{u}_{1}$ be the $p$-harmonic function in $\tilde{\Omega}_{1}=\left\{x: x_{n}>\psi\left(x^{\prime}\right)\right\}$ defined by Lemma 6.1. That is, $\tilde{\Omega}_{1}$ is obtained by adding the blip in (2.12) to $\Omega_{0}$ along $Q(1)$ and so $\partial \tilde{\Omega} \cap\left(P_{Q(1)} \cup \tilde{P}_{Q(1)}\right)=\partial \tilde{\Omega}_{1} \cap\left(P_{Q(1)} \cup \tilde{P}_{Q(1)}\right)$. We set

$$
\tilde{\lambda}=\frac{|\nabla \tilde{u}(\hat{z})|}{\left|\nabla \tilde{u}_{1}(\hat{z})\right|} \quad \text { for some } \hat{z} \in \partial \tilde{\Omega} \cap \partial \tilde{\Omega}_{1},|\hat{z}| \approx N^{-\gamma},
$$

and we note, using Theorem 3.9, l'Hôpital's rule, the interior Harnack inequality, and Lemma 3.6, that

$$
\tilde{\lambda} \approx \frac{\tilde{u}\left(e_{n}\right)}{\tilde{u}_{1}\left(e_{n}\right)} \approx\left(\frac{\tilde{\mu}(B(0,1))}{\tilde{\mu}_{1}(B(0,1))}\right)^{1 /(p-1)} .
$$

Furthermore, using (7.57), the fact that $\tilde{u}_{1}\left(e_{n}\right) \approx 1$, and Lemma 3.6(ii), we see that

$$
\tilde{\lambda} \approx(\tilde{\mu}(B(0,1)))^{1 /(p-1)} \approx \lambda .
$$

We also need a more refined estimate for $|\lambda-\tilde{\lambda}|$. In particular, using the point $\hat{z}$ and the triangle inequality we have

$$
|\lambda-\tilde{\lambda}| \leq|| \nabla u(0)|-| \nabla u(\hat{z})||+|| \nabla u(\hat{z})|-| \nabla \tilde{u}(\hat{z})||+\tilde{\lambda}|| \nabla \tilde{u}_{1}(\hat{z})|-1| .
$$


Since $Q(1)$ is part of a hyperplane, we see from Schwarz reflection and Schauder type arguments as in Section 6, Harnack's inequality, and Lemma 3.6(ii) that

$$
|| \nabla u(0)|-| \nabla u(\hat{z})|| \leq c|\hat{z}| \lambda \approx N^{-\gamma} \lambda .
$$

Furthermore, as in (7.68) we deduce from (7.55), (7.56) that

||$\nabla u(\hat{z})|-| \nabla \tilde{u}(\hat{z})|| \leq c|\nabla u(\hat{z})| \frac{\theta_{0}^{\alpha} N^{1-n} \lambda^{p-1}}{N^{(1-\gamma) \alpha} \mu\left(B\left(0, N^{-\gamma}\right)\right)} \leq c^{2} \lambda N^{-(1-\gamma)(\alpha+n-1)}$.

Finally, from Lemma 6.2 applied to $\Omega_{0}, \tilde{\Omega}_{1}$, and (7.75), we see that

$$
\tilde{\lambda}|| \nabla \tilde{u}_{1}(\hat{z})|-1| \leq c \lambda N^{-(1-\gamma)(\alpha+n-1)} .
$$

Combining (7.77)-(7.79) and using the result in (7.76) we can conclude that

$$
|\lambda-\tilde{\lambda}| \leq c\left(N^{-\gamma}+N^{-(1-\gamma)(\alpha+n-1)}\right) \lambda .
$$

Before we estimate $J$ we need one more preliminary estimate. In particular if $z \in$ $\partial \tilde{\Omega} \cap \partial \tilde{\Omega}_{1},|z| \leq N^{-\gamma}$, then we first note that

$$
\left|\tilde{\lambda}^{-1}\right| \nabla \tilde{u}(z)|-| \nabla \tilde{u}_{1}(z)||=\tilde{\lambda}^{-1}\left|\frac{|\nabla \tilde{u}(z)|}{\left|\nabla \tilde{u}_{1}(z)\right|}-\frac{|\nabla \tilde{u}(\hat{z})|}{\left|\nabla \tilde{u}_{1}(\hat{z})\right|}\right|\left|\nabla \tilde{u}_{1}(z)\right|,
$$

and second, from Theorem 3.9 with $r=1$, and l'Hôpital rule, we see that there exists $\bar{\sigma} \in(0,1)$ such that

$$
\left|\tilde{\lambda}^{-1}\right| \nabla \tilde{u}(z)|-| \nabla \tilde{u}_{1}(z)|| \leq \tilde{\lambda}^{-1} N^{-\gamma \bar{\sigma}} \frac{|\nabla \tilde{u}(\hat{z})|}{\left|\nabla \tilde{u}_{1}(\hat{z})\right|}\left|\nabla \tilde{u}_{1}(z)\right| \leq c N^{-\gamma \bar{\sigma}}\left|\nabla \tilde{u}_{1}(z)\right| .
$$

After these preliminaries we begin the estimate of $J$ in (7.59). In particular, we first introduce the decomposition

$$
J=J_{1}+J_{2}+J_{3}+J_{4},
$$

where

$$
\begin{aligned}
& J_{1}= \\
& \int_{\partial \tilde{\Omega} \cap B\left(0, N^{-\gamma}\right)}\left[\lambda^{-(p-1)}|\nabla \tilde{u}|^{p-1} \log \left(\lambda^{-1}|\nabla \tilde{u}|\right)-\tilde{\lambda}^{-(p-1)}|\nabla \tilde{u}|^{p-1} \log \left(\tilde{\lambda}^{-1}|\nabla \tilde{u}|\right)\right] d H^{n-1}, \\
& J_{2}=\int_{\partial \tilde{\Omega} \cap B\left(0, N^{-\gamma}\right)}\left[\tilde{\lambda}^{-(p-1)}|\nabla \tilde{u}|^{p-1} \log \left(\tilde{\lambda}^{-1}|\nabla \tilde{u}|\right)-\left|\nabla \tilde{u}_{1}\right|^{p-1} \log \left|\nabla \tilde{u}_{1}\right|\right] d H^{n-1}, \\
& J_{3}=\int_{\partial \tilde{\Omega} \cap B\left(0, N^{-\gamma}\right)}\left|\nabla \tilde{u}_{1}\right|^{p-1} \log \left|\nabla \tilde{u}_{1}\right| d H^{n-1}, \\
& J_{4}=-\int_{\partial \Omega \cap B\left(0, N^{-\gamma}\right)} \lambda^{-(p-1)}|\nabla u|^{p-1} \log \left(\lambda^{-1}|\nabla u|\right) d H^{n-1} .
\end{aligned}
$$


As for $J_{1}$ we note that

$$
\begin{aligned}
\left|J_{1}\right| \leq & \lambda^{-(p-1)}\left|\log \left(\lambda^{-1}\right)\right| \int_{\partial \tilde{\Omega} \cap B\left(0, N^{-\gamma}\right)}|\nabla \tilde{u}|^{p-1} d H^{n-1} \\
& +\left|\lambda^{-(p-1)}-\tilde{\lambda}^{-(p-1)}\right| \int_{\partial \tilde{\Omega} \cap B\left(0, N^{-\gamma}\right)}|\nabla \tilde{u}|^{p-1} \log \left(\tilde{\lambda}^{-1}|\nabla \tilde{u}|\right) d H^{n-1} \\
= & : J_{11}+J_{12} .
\end{aligned}
$$

Using (7.55), (7.57), and elementary estimates for the logarithm we see that

$$
J_{11} \leq c|\lambda-\tilde{\lambda}| \lambda^{-p} \tilde{\mu}\left(B\left(0, N^{-\gamma}\right)\right) \leq c|\lambda-\tilde{\lambda}| \lambda^{-1} N^{-\gamma(n-1)} .
$$

Hence, using (7.86)-(7.80), we can conclude that

$$
J_{11} \leq c\left(N^{-\gamma}+N^{-(1-\gamma)(\alpha+n-1)}\right) N^{-\gamma(n-1)}=c\left(N^{-\gamma n}+N^{-((1-\gamma) \alpha+n-1)}\right) .
$$

Considering the term $J_{12}$ we observe from (7.75) and (7.80) that

$$
\begin{aligned}
J_{12} & \leq|\lambda-\tilde{\lambda}| \lambda^{-p} \int_{\partial \tilde{\Omega} \cap B\left(0, N^{-\gamma}\right)}|\nabla \tilde{u}|^{p-1}\left|\log \left(\tilde{\lambda}^{-1}|\nabla \tilde{u}|\right)\right| d H^{n-1} \\
& \leq c\left(N^{-\gamma}+N^{-(1-\gamma)(\alpha+n-1)}\right) \lambda^{-(p-1)} J_{121}
\end{aligned}
$$

where

$$
J_{121}:=\int_{\partial \tilde{\Omega} \cap B\left(0, N^{-\gamma}\right)}|\nabla \tilde{u}|^{p-1}\left|\log \left(\tilde{\lambda}^{-1}|\nabla \tilde{u}|\right)\right| d H^{n-1} .
$$

To complete an estimate of $J_{12}$ we first estimate $J_{121}$. Indeed, using (3.8) we first see that

$$
J_{121} \leq c \tilde{\mu}\left(B\left(0, N^{-\gamma}\right)\right)\left|\log \left(c \tilde{\lambda}^{-1}\left(\frac{\tilde{\mu}\left(B\left(0, N^{-\gamma}\right)\right)}{H^{n-1}\left(B\left(0, N^{-\gamma}\right)\right)}\right)^{1 /(p-1)}\right)\right|
$$

Second, from (7.90), (7.79), (7.55), and (7.57), it follows that

$$
\begin{aligned}
J_{121} & \leq c \mu\left(B\left(0, N^{-\gamma}\right)\right)\left|\log \left(c \lambda^{-1}\left(\frac{\mu\left(B\left(0, N^{-\gamma}\right)\right)}{H^{n-1}\left(B\left(0, N^{-\gamma}\right)\right)}\right)^{1 /(p-1)}\right)\right| \\
& \leq c \lambda^{p-1} N^{-\gamma(n-1)} .
\end{aligned}
$$

Now using (7.91) in (7.88) we see that

$$
J_{12} \leq c\left(N^{-\gamma n}+N^{-((1-\gamma) \alpha+n-1)}\right) .
$$

Hence, from (7.92) and (7.87) we have

$$
J_{1} \leq J_{11}+J_{12} \leq c\left(N^{-\gamma n}+N^{-((1-\gamma) \alpha+n-1)}\right) .
$$


We next consider the term $J_{2}$. We write

$$
\begin{aligned}
J_{2} \leq & \int_{\partial \tilde{\Omega} \cap B\left(0, N^{-\gamma}\right)} \tilde{\lambda}^{-(p-1)}|\nabla \tilde{u}|^{p-1}\left|\log \left(\tilde{\lambda}^{-1}\left(\frac{|\nabla \tilde{u}|}{\left|\nabla \tilde{u}_{1}\right|}\right)\right)\right| d H^{n-1} \\
& +\int_{\partial \tilde{\Omega} \cap B\left(0, N^{-\gamma}\right)}\left|\left(\tilde{\lambda}^{-(p-1)}|\nabla \tilde{u}|^{p-1}-\left|\nabla \tilde{u}_{1}\right|^{p-1}\right) \log \left(\left|\nabla \tilde{u}_{1}\right|\right)\right| d H^{n-1} \\
= & : J_{21}+J_{22} .
\end{aligned}
$$

To estimate $J_{21}$ and $J_{22}$ we first note, using Theorem 3.9, that

$$
\left|\log \left(\tilde{\lambda}^{-1}\left(\frac{|\nabla \tilde{u}(z)|}{\left|\nabla \tilde{u}_{1}(z)\right|}\right)\right)\right| \leq c N^{-\gamma \bar{\sigma}} \quad \text { whenever }|z| \leq N^{-\gamma} .
$$

Furthermore, since

$$
\begin{aligned}
&\left.\left|\tilde{\lambda}^{-(p-1)}\right| \nabla \tilde{u}\right|^{p-1}-\left|\nabla \tilde{u}_{1}\right|^{p-1} \mid \\
& \leq\left. c\left|\tilde{\lambda}^{-1}\right| \nabla \tilde{u}|-| \nabla \tilde{u}_{1}||\left|\tilde{\lambda}^{-(p-2)}\right| \nabla \tilde{u}\right|^{p-2}+\left|\nabla \tilde{u}_{1}\right|^{p-2} \mid,
\end{aligned}
$$

we also see, using (7.82), Theorem 3.9 and the Harnack inequality for $p$-harmonic functions, that

$$
\left.\left|\tilde{\lambda}^{-(p-1)}\right| \nabla \tilde{u}(z)\right|^{p-1}-\left.\left|\nabla \tilde{u}_{1}(z)\right|^{p-1}\left|\leq c N^{-\gamma \bar{\sigma}}\right| \nabla \tilde{u}_{1}(z)\right|^{p-1}
$$

for $H^{n-1}$-almost every $z$ such that $|z| \leq N^{-\gamma}$. In particular, we can conclude that

$$
\begin{aligned}
& J_{21} \leq c N^{-\gamma \bar{\sigma}} \int_{\partial \tilde{\Omega} \cap B\left(0, N^{-\gamma}\right)} \tilde{\lambda}^{-(p-1)}|\nabla \tilde{u}|^{p-1} d H^{n-1} \\
& J_{22} \leq c N^{-\gamma \bar{\sigma}} \int_{\partial \tilde{\Omega} \cap B\left(0, N^{-\gamma}\right)}\left|\nabla \tilde{u}_{1}\right|^{p-1}|\log | \nabla \tilde{u}_{1}|| d H^{n-1} .
\end{aligned}
$$

Hence, using (7.55), (7.57), and (7.75) we see that

$$
J_{21} \leq c N^{-\gamma \bar{\sigma}} \lambda^{-(p-1)} \mu\left(B\left(0, N^{-\gamma}\right)\right) \leq c N^{-\gamma \bar{\sigma}} N^{-\gamma(n-1)}
$$

To estimate $J_{22}$ we use (7.98) and (3.8) to first get

$$
J_{22} \leq c N^{-\gamma \bar{\sigma}} \tilde{\mu}_{1}\left(B\left(0, N^{-\gamma}\right)\right)\left|\log \frac{\tilde{\mu}_{1}\left(B\left(0, N^{-\gamma}\right)\right)}{H^{n-1}\left(B\left(0, N^{-\gamma}\right)\right)}\right| .
$$

Second, from Lemma 7.2(a) applied to $\Omega_{0}, \tilde{\Omega}_{1}$, and Lemma 3.6(ii), we deduce that $\tilde{\mu}_{1}\left(B\left(0, N^{-\gamma}\right)\right) \approx N^{-\gamma(n-1)}$. Using this inequality in (7.100) we find that

$$
J_{22} \leq c N^{-\gamma \bar{\sigma}} N^{-\gamma(n-1)} .
$$

Putting (7.101), (7.99) together we have

$$
J_{2} \leq J_{21}+J_{22} \leq c N^{-\gamma \bar{\sigma}} N^{-\gamma(n-1)},
$$

and this completes our estimate of the term $J_{2}$. 
We next consider the term $J_{3}$. Now

$$
J_{3}=I(\psi, p)-\int_{\mathbb{R}^{n-1} \backslash B\left(0, N^{-\gamma}\right)}\left|\nabla \tilde{u}_{1}\right|^{p-1} \log \left|\nabla \tilde{u}_{1}\right| d H^{n-1} .
$$

To estimate the last integral note from Lemma 7.2(b), again applied to $\Omega_{0}, \tilde{\Omega}_{1}$, and l'Hôpital's rule, that

$$
|\log | \nabla u_{1}(x)|| \leq \frac{c \theta_{0}^{\alpha} N^{1-n}}{N^{\alpha}|x|^{n-1+\alpha}} \quad \text { whenever }|x| \geq 100 / N .
$$

Using this inequality to estimate the integral in (7.103) we see that

$$
\left|J_{3}-I(\psi, p)\right| \leq c N^{-((1-\gamma) \alpha+n-1)} .
$$

Finally, we consider $J_{4}$. Using (3.8) and (7.55) we get

$$
\begin{aligned}
J_{4} & \leq c \lambda^{-(p-1)} \mu\left(B\left(0, N^{-\gamma}\right)\right)\left|\log \left(\lambda^{-1}\left(\frac{\mu\left(B\left(0, N^{-\gamma}\right)\right)}{H^{n-1}\left(B\left(0, N^{-\gamma}\right)\right)}\right)^{1 /(p-1)}\right)\right| \\
& \leq c N^{-\gamma n} .
\end{aligned}
$$

Collecting the estimates in (7.93), (7.102), (7.104), (7.105), we have proved that

$$
\begin{aligned}
|\hat{I}(R)-I(\psi, p)| & \leq\left|J_{1}\right|+\left|J_{2}\right|+\left|J_{4}\right|+\sum_{k=1}^{\infty}\left|I_{k}\right|+c N^{-((1-\gamma) \alpha+n-1)} \\
& \leq c\left(N^{-\gamma n}+N^{-((1-\gamma) \alpha+(n-1))}+N^{-\gamma \bar{\sigma}} N^{-\gamma(n-1)}\right),
\end{aligned}
$$

where $\alpha, \bar{\sigma} \in(0,1)$ are fixed numbers while $\gamma \in(0,1)$ is a degree of freedom. To complete the argument, and the proof, we now let

$$
\gamma=\min \left\{1-\frac{1}{2 n}, \frac{1}{2}\left(1+\frac{n-1}{\bar{\sigma}+n-1}\right)\right\} .
$$

Then we can conclude from (7.106), (7.107) that there exists $\beta=\beta(p, n)>0$ such that

$$
\hat{I}(R) \leq I(\psi, p)+c N^{1-n-\beta} .
$$

Moreover, $\beta$ can be chosen independent of $p$ when $p \in[3 / 2,5 / 2]$. Letting $R \rightarrow \infty$ and using Lemma 7.7, (7.54), (7.58), it follows from (7.108) that

$$
\begin{aligned}
& \lim _{R \rightarrow \infty} t^{n-1} \lambda^{p-1} \hat{I}(R) \\
& \quad=\left(\int_{\partial \Omega_{k}^{j+1}}\left|\nabla u_{k}^{j+1}\right|^{p-1} \log \left|\nabla u_{k}^{j+1}\right| d H^{n-1}-\int_{\partial \Omega_{k}^{j}}\left|\nabla u_{k}^{j}\right|^{p-1} \log \left|\nabla u_{k}^{j}\right| d H^{n-1}\right),
\end{aligned}
$$

where $t=8^{-k-1}$, and that

$$
\lim _{R \rightarrow \infty} t^{n-1} \lambda^{p-1} \hat{I}(R) \leq t^{n-1} \lambda^{p-1}\left(I(\psi, p)+c N^{1-n-\beta}\right) .
$$


Furthermore, from (7.54), (7.55), we deduce that

$$
t^{n-1} \lambda^{p-1} \approx N^{n-1} \mu_{k}^{j}\left(B\left(a_{Q_{j}}, t / N\right)\right) .
$$

Using this inequality in the second last display we conclude that the proof of Lemma 7.8 is complete.

Acknowledgments. Lewis was partially supported by NSF DMS-0900291. Nyström was partially supported by grant VR-70629701.

\section{References}

[Ba] Batakis, A.: Harmonic measure of some Cantor type sets. Ann. Acad. Sci. Fenn. Math. 21, 255-270 (1996) Zbl 0849.31005 MR 1404086

[BL] Bennewitz, B., Lewis, J.: On the dimension of $p$-harmonic measure. Ann. Acad. Sci. Fenn. Math. 30, 459-505 (2005) Zbl 1194.35189 MR 2173375

[Bo] Bourgain, J.: On the Hausdorff dimension of harmonic measure in higher dimensions. Invent. Math. 87, 477-483 (1987) Zbl 0616.31004 MR 0874032

[CKL] Capogna, L., Kenig, C., Lanzani, L.: Harmonic Measure-Geometric and Analytic Points of View. Univ. Lecture Ser. 35, Amer. Math. Soc. (2005) Zbl 1074.31001 MR 2139304

[C] Carleson, L.: On the support of harmonic measure for sets of Cantor type. Ann. Acad. Sci. Fenn. 10, 113-123 (1985) Zbl 0593.31004 MR 0802473

[D] Dahlberg, B.: Estimates of harmonic measure. Arch. Rat. Mech. Anal. 65, 275-288 (1977) Zbl 0406.28009 MR 0466593

[DJK] Dahlberg, B., Jerison, D., Kenig, C.: Area integral estimates for elliptic differential operators with nonsmooth coefficients. Ark. Mat. 22, 97-108 (1984) Zbl 0537.35025 MR 0735881

[FJK1] Fabes, E., Jerison, D., Kenig, C.: The Wiener test for degenerate elliptic equations. Ann. Inst. Fourier (Grenoble) 32, 151-182 (1982) Zbl 0488.35034 MR 0688024

[FJK2] Fabes, E., Jerison, D., Kenig, C.: Boundary behavior of solutions to degenerate elliptic equations. In: Conference on Harmonic Analysis in Honor of Antoni Zygmund, Vol. I, II (Chicago, IL, 1981), Wadsworth Math. Ser., Wadsworth, Belmont, CA, 577-589 (1983) Zbl 0503.35038 MR 0730093

[FKS] Fabes, E., Kenig, C., Serapioni, R.: The local regularity of solutions to degenerate elliptic equations. Comm. Partial Differential Equations 7, 77-116 (1982) Zbl 0498.35042 MR 0643158

[GT] Gilbarg, D., Trudinger, N. S.: Elliptic Partial Differential Equations of Second Order. 2nd ed., Springer (1983) Zbl 0562.35001 MR 0737190

[HKM] Heinonen, J., Kilpeläinen, T., Martio, O.: Nonlinear Potential Theory of Degenerate Elliptic Equations. Oxford Univ. Press (1993) Zbl 0780.31001 MR 1207810

[JK] Jerison, D., Kenig, C.: Boundary behavior of harmonic functions in non-tangentially accessible domains. Adv. Math. 46, 80-147 (1982) Zbl 0514.31003 MR 0676988

[JW] Jones, P., Wolff, T.: Hausdorff dimension of harmonic measure in the plane. Acta Math. 161, 131-144 (1988) Zbl 0667.30020 MR 0962097

[KW] Kaufmann, R., Wu, J. M.: On the snowflake domain. Ark. Mat. 23, 177-183 (1985) MR 0800179

[KT] Kenig, C., Toro, T.: Harmonic measure on locally flat domains. Duke Math. J. 87, 509-551 (1997) Zbl 0878.31002 MR 1446617 
[KPT] Kenig, C., Preiss, D., Toro, T.: Boundary structure and size in terms of interior and exterior harmonic measures in higher dimension. J. Amer. Math. Soc 22, 771-796 (2009) Zbl 1206.28002 MR 2505300

[L] Lewis, J.: Note on $p$ harmonic measure. Comput. Methods Function Theory 6, 109-144 (2006) Zbl 1161.35406 MR 2241036

[LLuN] Lewis, J., Lundström, N., Nyström, K.: Boundary Harnack inequalities for operators of $p$-Laplace type in Reifenberg flat domains. In: Perspectives in PDE, Harmonic Analysis, and Applications, Proc. Sympos. Pure Math. 79, Amer. Math. Soc., 229-266 (2008) Zbl 1160.35423 MR 2500495

[LN1] Lewis, J., Nyström, K.: Boundary behaviour for $p$-harmonic functions in Lipschitz and starlike Lipschitz ring domains. Ann. Sci. École Norm. Sup. 40, 765-813 (2007) Zbl 1134.31008 MR 2382861

[LN2] Lewis, J., Nyström, K.: Boundary behaviour and the Martin boundary problem for $p$-harmonic functions in Lipschitz domains. Ann. of Math. 172, 1907-1948 (2010) Zbl 1210.31004 MR 2726103

[LN3] Lewis, J., Nyström, K.: Regularity and free boundary regularity for the $p$-Laplacian in Lipschitz and $C^{1}$-domains. Ann. Acad. Sci. Fenn. Math. 33, 523-548 (2008) Zbl 1202.35110 MR 2431379

[LN4] Lewis, J., Nyström, K.: New results for $p$-harmonic functions. Pure Appl. Math. Quart. 7, 345-363 (2011) Zbl 1241.35110 MR 2815383

[LN5] Lewis, J., Nyström, K.: Boundary behaviour of $p$-harmonic functions in domains beyond Lipschitz domains. Adv. Calc. Var. 1, 133-177 (2008) Zbl 1169.31004 MR 2427450

[LN6] Lewis, J., Nyström, K.: Regularity of Lipschitz free boundaries in two-phase problems for the $p$-Laplace operator. Adv. Math. 225, 2565-2597 (2010) Zbl 1200.35335 MR 2680176

[LNP] Lewis, J., Nyström, K., Poggi-Corradini, P.: p-harmonic measure in simply-connected domains. Ann. Inst. Fourier (Grenoble) 61, 689-715 (2011) Zbl 1241.35071 MR 2895070

[LVV] Lewis, J., Verchota, G., Vogel, A.: On Wolff snowflakes. Pacific J. Math. 218, 139-166 (2005) Zbl 1108.31006 MR 2224593

[Li] Lieberman, G. M.: Boundary regularity for solutions of degenerate elliptic equations. Nonlinear Anal. 12, 1203-1219 (1988) Zbl 0675.35042 MR 0969499

[M] Makarov, N.: On the distortion of boundary sets under conformal mappings. Proc. London Math. Soc. 51, 369-384 (1985) Zbl 0573.30029 MR 0794117

[P] Pommerenke, C.: Boundary Behaviour of Conformal Maps. Grundlehren Math. Wiss. 299, Springer (1992) Zbl 0762.30001 MR 1217706

[R] Reshetnyak, Y. G.: Space Mappings with Bounded Distortion. Transl. Math. Monogr. 73, Amer. Math. Soc. (1989) Zbl 0667.30018 MR 0994644

[RN] Riesz, F., Sz.-Nagy, B.: Functional Analysis. Dover Publ. (1990) Zbl 0732.47001 MR 1068530

[S] Stein, E.: Harmonic Analysis. Princeton Univ. Press (1993) Zbl 0821.42001 MR 1232192

[V] Volberg, A.: On the dimension of harmonic measure of Cantor repellers. Michigan Math. J. 40, 239-258 (1993) Zbl 0797.30022 MR 1226830

[W1] Wolff, T.: Plane harmonic measures live on sets of finite linear length. Ark. Mat. 31, 137172 (1993) Zbl 0809.30007 MR 1230270

[W2] Wolff, T.: Counterexamples with harmonic gradients in $\mathbb{R}^{3}$. In: Essays on Fourier Analysis in Honor of Elias M. Stein, Princeton Math. Ser. 42, Princeton Univ. Press, 321-384 (1995) Zbl 0836.31004 MR 1315554 